\title{
Vietnam: Statistical Appendix
}

This Statistical Appendix for Vietnam was prepared by a staff team of the International Monetary Fund as background documentation for the periodic consultation with the member country. It is based on the information available at the time it was completed on September 28, 2006. The views expressed in this document are those of the staff team and do not necessarily reflect the views of the government of Vietnam or the Executive Board of the IMF.

The policy of publication of staff reports and other documents by the IMF allows for the deletion of market-sensitive information.

To assist the IMF in evaluating the publication policy, reader comments are invited and may be sent by e-mail to publicationpolicy@imf.org.

Copies of this report are available to the public from

International Monetary Fund • Publication Services

700 19th Street, N.W. • Washington, D.C. 20431

Telephone: (202) 6237430 • Telefax: (202) 6237201

E-mail: publications@imf.org • Internet: http://www.imf.org

Price: $\$ 18.00$ a copy

\section{International Monetary Fund Washington, D.C.}





\section{INTERNATIONAL MONETARY FUND}

\section{VIETNAM}

\section{Statistical Appendix}

Prepared by Carol Baker and Patrizia Tumbarello (both APD), and Faisal Ahmed (PDR)

Approved by the Asia and Pacific Department

September 28, 2006

Contents

Page

Basic Data 3

1. Gross Domestic Product by Expenditure Categories at Current Prices, 2001-05 ............4

2. Gross Domestic Product by Sector and Ownership at Current Prices, 2001-05 .............5

3. Gross Domestic Product by Sector at Constant Prices, 2001-05 ...................................6

4. Consumer Price Inflation, 2001-06 …………..........................................................

5. Gross Value of Agricultural Production at Constant Prices, 2001-05 …………............. 8

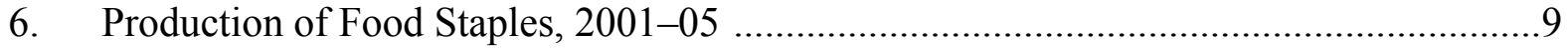

7. Industrial Crop Production and Livestock, 2001-05 .................................................10

8. Gross Value of Industrial Production at Constant Prices, 2001-05 ……….................11

9. Industrial Production by Sector of Ownership at Constant Prices, 2001-05 .................12

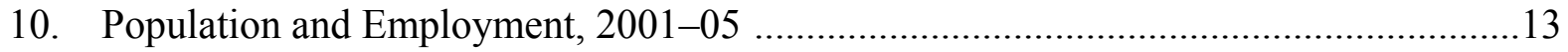

11. Total and Nonstate Employment by Sector, 2001-05 ..................................................

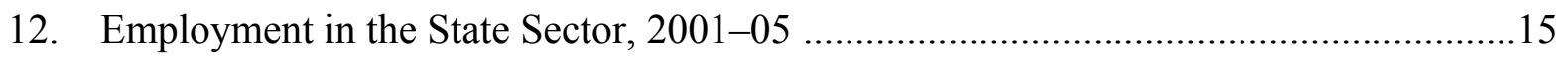

13. Summary of General Government Budgetary Operations, 2001-06 .............................16

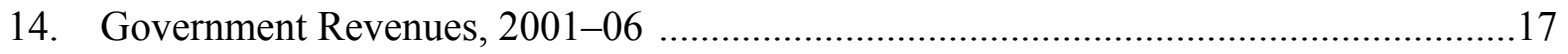

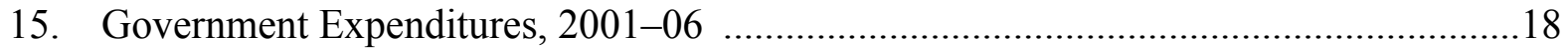

16. Treasury Bill and Bond Issues, 2004-06 ............................................................19

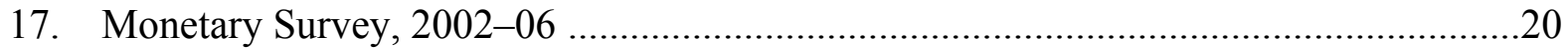

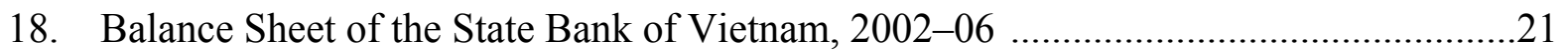

19. Consolidated Balance Sheet of Deposit Money Banks, 2002-06 ...............................22

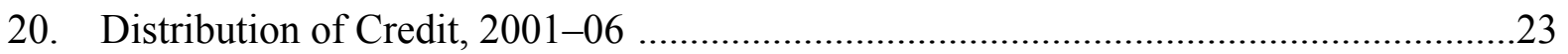

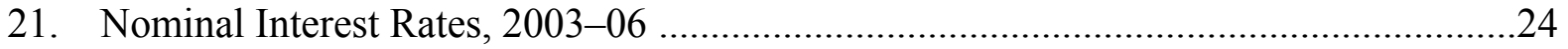

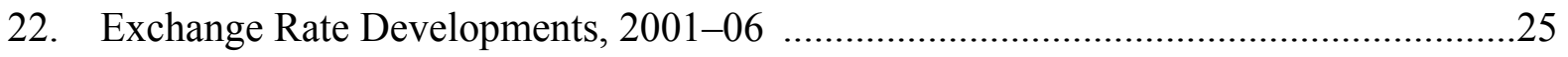

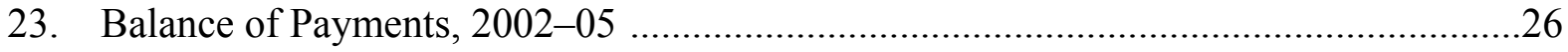

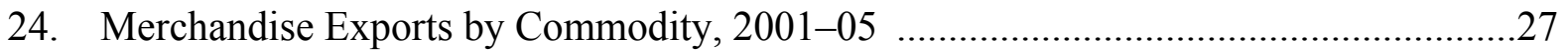

25. Merchandise Imports by Commodity, 2001-05 .......................................................28 
26. Direction of Trade, 2001-05

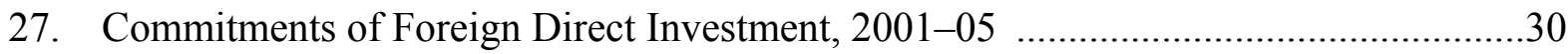

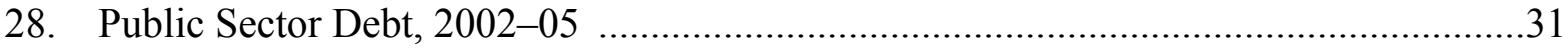

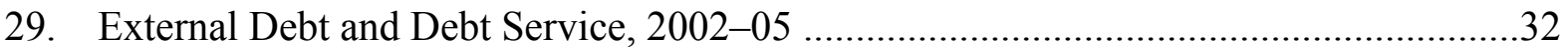

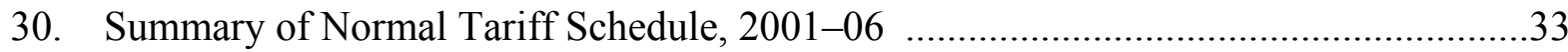

31. Common Effective Preferential Tariff (CEPT) Rates, 2001-06 ....................................34

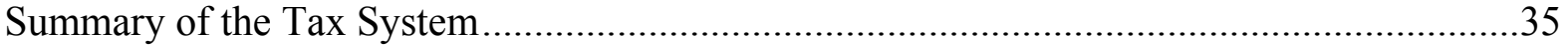


Vietnam: Basic Data

Nominal GDP (2005): US $\$ 52.8$ billion

GDP per capita (2005): US\$636 Population (2005): 83.12 million

Fund quota: SDR 329.1 million

\begin{tabular}{|c|c|c|c|c|c|}
\hline & 2001 & 2002 & 2003 & 2004 & 2005 \\
\hline Real GDP (annual percentage change) & 6.9 & 7.1 & 7.3 & 7.8 & 8.4 \\
\hline Industrial output & 14,6 & 14,8 & 16,8 & 16,6 & 17.2 \\
\hline Saving-investment balance (in percent of GDP) & 1.6 & -1.9 & -4.9 & -3.4 & 0.4 \\
\hline Gross national saving & 32.8 & 31.3 & 30.6 & 32.3 & 35.6 \\
\hline Private & 26.9 & 23.9 & 23.3 & 23.9 & 28.9 \\
\hline Public & 5.9 & 7.4 & 7.3 & 8.4 & 6.7 \\
\hline Gross investment & 31.2 & 33.2 & 35.4 & 35.7 & 35.2 \\
\hline Private & 20.6 & 21.5 & 20.8 & 23.1 & 22.0 \\
\hline Public & 10.6 & 11.7 & 14.7 & 12.6 & 13.2 \\
\hline \multicolumn{6}{|l|}{ Inflation (annual percentage change) } \\
\hline Period average & -0.4 & 4.0 & 3.2 & 7.7 & 8.3 \\
\hline End of period & 0.7 & 4.0 & 2.9 & 9.7 & 8.8 \\
\hline GDP deflator & 1.9 & 4.0 & 6.7 & 8.2 & 8.0 \\
\hline \multicolumn{6}{|l|}{ General government } \\
\hline Official budget balance & -2.8 & -1.4 & -1.2 & 0.9 & -1.2 \\
\hline Revenue and grants & 21.6 & 22.7 & 24.9 & 26.7 & 25.9 \\
\hline of which: oil revenue & 7.4 & 6.8 & 7.0 & 7.9 & 8.8 \\
\hline Expenditure & 24.4 & 24.2 & 26.1 & 25.8 & 27.1 \\
\hline Off-budget expenditure and net lending & 2.2 & 3.3 & 5.2 & 3.7 & 4.7 \\
\hline Net lending & 2.2 & 2.4 & 3.2 & 2.4 & 2.7 \\
\hline ODA & 1.0 & 0.9 & 1.5 & 1.4 & 0.8 \\
\hline DAF & 1.2 & 1.5 & 1.7 & 1.0 & 0.5 \\
\hline Sovereign bond-financed lending to SOEs & 0.0 & 0.0 & 0.0 & 0.0 & 1.4 \\
\hline Off-budget investment expenditure & 0.0 & 0.9 & 2.0 & 1.3 & 2.0 \\
\hline Overall fiscal balance including off-budget expenditure & -5.0 & -4.7 & -6.4 & -2.8 & -5.9 \\
\hline Non-oil overall fiscal deficit & -12.4 & -11.5 & -13.5 & -10.7 & -14.6 \\
\hline \multicolumn{6}{|l|}{ Money and credit (annual percentage change, end of period) } \\
\hline Broad money & 25.5 & 17.6 & 24.9 & 29.5 & 29.7 \\
\hline Credit to the economy & 21.4 & 22.2 & 28.4 & 41.6 & 31.7 \\
\hline Reserve money & 16.7 & 12.4 & 27.4 & 16.1 & 23.7 \\
\hline \multicolumn{6}{|l|}{ Interest rates (in percent, end of period) } \\
\hline Nominal three-month deposit rate (households) & 5.9 & 7.0 & 6.3 & 6.7 & 7.8 \\
\hline Nominal short-term lending rate (less than one year) & 8.8 & 9.9 & 10.0 & 10.7 & 12.0 \\
\hline Real three-month deposits rate (households) & 5.1 & 2.8 & 3.3 & -2.7 & -0.9 \\
\hline Real short-term lending rate (less than one year) & 8.0 & 5.6 & 6.9 & 1.0 & 3.0 \\
\hline \multicolumn{6}{|l|}{ Current account balance (including official transfers) } \\
\hline (in millions of U.S. dollars) & 524 & -673 & $-1,932$ & $-1,565$ & 218 \\
\hline (in percent of GDP) & 1.6 & -1.9 & -4.9 & -3.4 & 0.4 \\
\hline Exports f.o.b (annual percentage change, U.S. dollar terms) & 4.0 & 11.2 & 20.6 & 31.4 & 22.5 \\
\hline Imports f.o.b. (annual percentage change, U.S. dollar terms) & 3.4 & 22.1 & 28.0 & 26.6 & 15.7 \\
\hline \multicolumn{6}{|l|}{ Foreign exchange reserves (in millions of U.S. dollars, end of period) } \\
\hline Gross official reserves, including gold & 3,387 & 3,692 & 5,620 & 6,314 & 8,557 \\
\hline (in weeks of next year's imports of goods and nonfactor services) & 8.2 & 7.2 & 8.7 & 8.5 & 9.8 \\
\hline External debt (in percent of GDP) 1/ & 38.5 & 35.2 & 34.2 & 33.9 & 32.0 \\
\hline Total public and publicly guaranteed debt (in percent of GDP) $1 /$ & 36.0 & 38.2 & 41.1 & 42.7 & 44.0 \\
\hline \multicolumn{6}{|l|}{ Exchange rate (dong per U.S. dollar) } \\
\hline Period average $2 /$ & 14,786 & 15,244 & 15,475 & 15,704 & 15,816 \\
\hline End of period $2 /$ & 15,070 & 15,368 & 15,608 & 15,739 & 15,875 \\
\hline \multicolumn{6}{|l|}{ Real effective exchange rate (annual percentage) $3 /$} \\
\hline Period average & 0.1 & -1.8 & -7.9 & -1.4 & 4.3 \\
\hline End of period & 0.4 & -5.5 & -9.0 & 1.1 & 13.0 \\
\hline \multicolumn{6}{|l|}{ Memorandum items: } \\
\hline GDP (in trillions of dong at current market prices) & 481.3 & 535.8 & 613.4 & 715.3 & 837.9 \\
\hline Per capita GDP (in U.S. dollars) & 413 & 440 & 489 & 554 & 636 \\
\hline
\end{tabular}

Sources: Data provided by the authorities; and Fund staff estimates and projections.

1/ Debt data are not fully comparable to the data in the staff report due to revisions as of September 20, 2006.

2/ Interbank market rate.

3/ A positive number implies an appreciation. 
Table 1. Vietnam: Gross Domestic Product by Expenditure Categories at Current Prices, 2001-05 1/

\begin{tabular}{|c|c|c|c|c|c|}
\hline & 2001 & 2002 & 2003 & 2004 & $\frac{2005}{\text { Est. }}$ \\
\hline & \multicolumn{5}{|c|}{ (In billions of dong) } \\
\hline Consumption & 342,607 & 382,137 & 445,221 & 511,221 & 548,793 \\
\hline Private & 312,144 & 348,747 & 406,451 & 465,506 & 533,141 \\
\hline Government & 30,463 & 33,390 & 38,770 & 45,715 & 51,652 \\
\hline Investment & 150,033 & 177,983 & 217,434 & 253,686 & 297,000 \\
\hline Gross capital formation & 140,301 & 166,828 & 204,608 & 237,868 & 277,547 \\
\hline Change in stocks & 9,732 & 11,155 & 12,826 & 15,818 & 19,453 \\
\hline Domestic demand & 492,640 & 560,120 & 662,655 & 764,907 & 881,793 \\
\hline Net exports & $-10,982$ & $-27,684$ & $-51,288$ & $-54,000$ & $-38,469$ \\
\hline Exports & 262,846 & 304,262 & 363,735 & 470,216 & 578,373 \\
\hline Imports & 273,828 & 331,946 & 415,023 & 524,216 & 616,842 \\
\hline Statistical discrepancy 2/ & -363 & 3,326 & 2,076 & 4,400 & $-5,466$ \\
\hline \multirow[t]{2}{*}{ GDP } & 481,295 & 535,762 & 613,443 & 715,307 & 837,858 \\
\hline & \multicolumn{5}{|c|}{ (In percent of GDP) } \\
\hline Consumption & 71.2 & 71.3 & 72.6 & 71.5 & 69.8 \\
\hline Private & 64.9 & 65.1 & 66.3 & 65.1 & 63.6 \\
\hline Government & 6.3 & 6.2 & 6.3 & 6.4 & 6.2 \\
\hline Investment & 31.2 & 33.2 & 35.4 & 35.5 & 35.4 \\
\hline Gross capital formation & 29.2 & 31.1 & 33.4 & 33.3 & 33.1 \\
\hline Change in stocks & 2.0 & 2.1 & 2.1 & 2.2 & 2.3 \\
\hline Domestic demand & 102.4 & 104.5 & 108.0 & 106.9 & 105.2 \\
\hline Net exports & -2.3 & -5.2 & -8.4 & -7.5 & -4.6 \\
\hline Exports & 54.6 & 56.8 & 59.3 & 65.7 & 69.0 \\
\hline Imports & 56.9 & 62.0 & 67.7 & 73.3 & 73.6 \\
\hline \multirow[t]{2}{*}{ Statistical discrepancy 2/ } & -0.1 & 0.6 & 0.3 & 0.6 & -0.7 \\
\hline & \multicolumn{5}{|c|}{ (Annual percentage change) } \\
\hline Consumption & 6.4 & 11.5 & 16.5 & 14.8 & 14.4 \\
\hline Private & 6.3 & 11.7 & 16.5 & 14.5 & 14.5 \\
\hline Government & 7.5 & 9.6 & 16.1 & 17.9 & 13.0 \\
\hline Investment & 14.7 & 18.6 & 22.2 & 16.7 & 17.1 \\
\hline Gross capital formation & 14.9 & 18.9 & 22.6 & 16.3 & 16.7 \\
\hline Domestic demand & 8.8 & 13.7 & 18.3 & 15.4 & 15.3 \\
\hline Exports & 8.1 & 15.8 & 19.5 & 29.3 & 23.0 \\
\hline Imports & 7.8 & 21.2 & 25.0 & 26.3 & 17.7 \\
\hline \multirow[t]{2}{*}{ GDP } & 9.0 & 11.3 & 14.5 & 16.6 & 17.1 \\
\hline & \multicolumn{5}{|c|}{ (Contribution to nominal GDP growth, in percent) } \\
\hline Domestic demand & 100.9 & 123.9 & 132.0 & 100.4 & 95.4 \\
\hline Private consumption & 52.3 & 72.6 & 81.2 & 64.8 & 60.0 \\
\hline Fixed capital formation & 45.9 & 48.7 & 48.6 & 32.7 & 32.4 \\
\hline Change in stocks & 2.7 & 2.6 & 2.2 & 2.9 & 3.0 \\
\hline Net exports & -0.3 & -30.7 & -30.4 & -2.7 & 12.7 \\
\hline Statistical discrepancy 2/ & -0.7 & 6.8 & -1.6 & 2.3 & -8.1 \\
\hline GDP & 100.0 & 100.0 & 100.0 & 100.0 & 100.0 \\
\hline
\end{tabular}

Source: General Statistics Office.

1/ Data are latest official revisions of historical GDP estimates.

2/ Difference between production- and expenditure-based estimates of GDP, the former of which are considered to be more accurate. 
Table 2. Vietnam: Gross Domestic Product by Sector and Ownership at Current Prices, 2001-05 1/

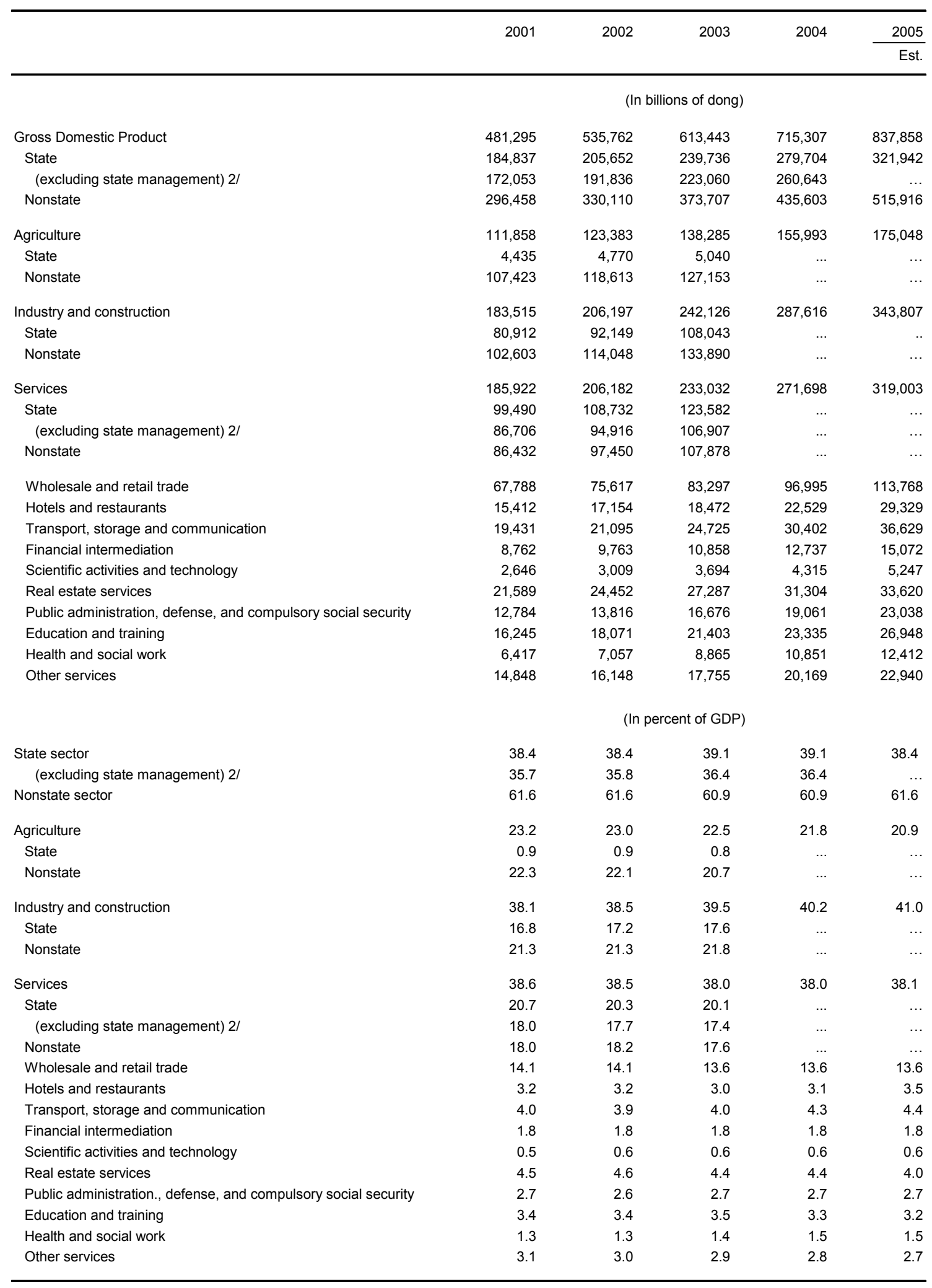

Source: General Statistics Office.

1/ Data are latest official revisions of historical GDP estimates.

2/ State management includes public administration, defense, and compulsory social security. 
Table 3. Vietnam: Gross Domestic Product by Sector at Constant Prices, 2001-05 1/

\begin{tabular}{|c|c|c|c|c|c|}
\hline & 2001 & 2002 & 2003 & 2004 & 2005 \\
\hline & \multicolumn{5}{|c|}{ (In billions of dong, at 1994 constant prices) } \\
\hline Agriculture, forestry, and fishery & 65,618 & 68,352 & 70,827 & 73,917 & 76,905 \\
\hline Agriculture & 55,613 & 57,912 & 59,761 & 62,107 & 64,033 \\
\hline Forestry & 2,556 & 2,568 & 2,589 & 2,610 & 2,640 \\
\hline Fishery & 7,449 & 7,872 & 8,477 & 9,200 & 10,232 \\
\hline Industry & 106,986 & 117,125 & 129,399 & 142,621 & 157,808 \\
\hline Mining and quarrying & 19,185 & 19,396 & 20,611 & 22,437 & 22,643 \\
\hline Manufacturing & 57,335 & 63,983 & 71,363 & 79,116 & 89,514 \\
\hline Electricity, gas, and water supply & 7,173 & 7,992 & 8,944 & 10,015 & 11,241 \\
\hline Construction & 23,293 & 25,755 & 28,481 & 31,053 & 34,410 \\
\hline Services & 119,931 & 127,770 & 136,016 & 145,897 & 158,277 \\
\hline Wholesale and retail trade & 47,779 & 51,245 & 54,747 & 59,027 & 63,957 \\
\hline Hotels and restaurants & 9,458 & 10,125 & 10,646 & 11,511 & 13,466 \\
\hline Transport, storage, and communications & 11,441 & 12,252 & 12,925 & 13,975 & 15,318 \\
\hline Financial intermediation & 6,005 & 6,424 & 6,935 & 7,495 & 8,197 \\
\hline Science and technology & 1,749 & 1,909 & 2,044 & 2,196 & 2,368 \\
\hline Real estate services & 12,631 & 13,106 & 13,796 & 14,396 & 14,809 \\
\hline State management $1 /$ & 8,439 & 8,768 & 9,228 & 9,773 & 10,477 \\
\hline Education and training & 9,687 & 10,475 & 11,260 & 12,125 & 13,126 \\
\hline Health and social work & 4,151 & 4,464 & 4,853 & 5,234 & 5,640 \\
\hline Other services & 8,591 & 9,002 & 9,582 & 10,165 & 10,918 \\
\hline \multirow[t]{2}{*}{ Gross Domestic Product } & 292,535 & 313,247 & 336,242 & 362,435 & 392,989 \\
\hline & \multicolumn{5}{|c|}{ (Real GDP, annual percentage change) } \\
\hline Agriculture, forestry, and fishery & 3.0 & 4.2 & 3.6 & 4.4 & 4.0 \\
\hline Agriculture & 2.1 & 4.1 & 3.2 & 3.9 & 3.1 \\
\hline Forestry & 0.5 & 0.5 & 0.8 & 0.8 & 1.1 \\
\hline Fishery & 11.5 & 5.7 & 7.7 & 8.5 & 11.2 \\
\hline Industry & 10.4 & 9.5 & 10.5 & 10.2 & 10.6 \\
\hline Mining and quarrying & 4.1 & 1.1 & 6.3 & 8.9 & 0.9 \\
\hline Manufacturing & 11.3 & 11.6 & 11.5 & 10.9 & 13.1 \\
\hline Electricity, gas, and water supply & 13.2 & 11.4 & 11.9 & 12.0 & 12.2 \\
\hline Construction & 12.8 & 10.6 & 10.6 & 9.0 & 10.8 \\
\hline Services & 6.1 & 6.5 & 6.5 & 7.3 & 8.5 \\
\hline Wholesale and retail trade & 7.0 & 7.3 & 6.8 & 7.8 & 8.4 \\
\hline Hotels and restaurants & 6.7 & 7.1 & 5.1 & 8.1 & 17.0 \\
\hline Transport, storage, and communications & 6.6 & 7.1 & 5.5 & 8.1 & 9.6 \\
\hline Financial intermediation & 6.3 & 7.0 & 8.0 & 8.1 & 9.4 \\
\hline Real estate services & 3.3 & 3.8 & 5.3 & 4.4 & 2.9 \\
\hline State management $1 /$ & 5.2 & 3.9 & 5.2 & 5.9 & 7.2 \\
\hline Education and training & 5.7 & 8.1 & 7.5 & 7.7 & 8.3 \\
\hline Health and social work & 5.2 & 7.5 & 8.7 & 7.9 & 7.7 \\
\hline Other services & 4.5 & 4.8 & 6.4 & 6.1 & 7.4 \\
\hline \multirow[t]{2}{*}{ Gross Domestic Product } & 6.9 & 7.1 & 7.3 & 7.8 & 8.4 \\
\hline & \multicolumn{5}{|c|}{ (GDP deflator, annual percentage change) } \\
\hline Agriculture, forestry, and fishery & 0.2 & 5.9 & 8.2 & 8.1 & 7.9 \\
\hline Agriculture & -1.7 & 5.5 & 6.8 & 7.7 & 8.0 \\
\hline Forestry & 2.6 & 6.2 & 18.6 & 20.1 & 5.6 \\
\hline Fishery & 7.7 & 7.5 & 10.1 & 4.9 & 5.9 \\
\hline Industry & 2.5 & 2.6 & 6.3 & 7.8 & 8.0 \\
\hline Mining and quarrying & 0.0 & 2.9 & 16.9 & 16.2 & 20.5 \\
\hline Manufacturing & 4.3 & 3.8 & 2.0 & 4.6 & 5.4 \\
\hline Electricity, gas, and water supply & 1.2 & 1.9 & 9.1 & 0.8 & 2.7 \\
\hline Construction & 4.8 & 2.2 & 6.3 & 10.2 & 7.9 \\
\hline Services & 2.4 & 4.1 & 6.2 & 8.7 & 8.2 \\
\hline Wholesale and retail trade & 0.8 & 4.0 & 3.1 & 8.0 & 8.3 \\
\hline Hotels and restaurants & 0.7 & 4.0 & 2.4 & 12.8 & 11.3 \\
\hline Transport, storage, and communications & 5.1 & 1.4 & 11.1 & 13.7 & 9.9 \\
\hline Financial intermediation & 1.2 & 4.2 & 3.0 & 8.6 & 8.2 \\
\hline Real estate services & 9.0 & 9.2 & 6.0 & 9.9 & 4.4 \\
\hline State management $1 /$ & 0.7 & 4.0 & 14.7 & 7.9 & 12.7 \\
\hline Education and training & 3.5 & 2.9 & 10.2 & 1.2 & 6.7 \\
\hline Health and social work & 1.7 & 2.3 & 15.6 & 13.5 & 6.2 \\
\hline Other services & 1.6 & 3.8 & 3.3 & 7.1 & 5.9 \\
\hline Gross Domestic Product & 1.9 & 4.0 & 6.7 & 8.2 & 8.0 \\
\hline
\end{tabular}

Sources: General Statistics Office; and Fund staff estimates.

$1 /$ State management includes public administration, defense, and compulsory social security. 
Table 4. Vietnam: Consumer Price Inflation, 2001-06 1/ (Index, 2001=100, unless otherwise indicated)

\begin{tabular}{|c|c|c|c|c|c|c|c|c|c|}
\hline \multirow[b]{3}{*}{ Weight (in percent) } & \multicolumn{2}{|c|}{ Overall index } & \multicolumn{2}{|c|}{ Food } & \multicolumn{5}{|c|}{ Nonfood } \\
\hline & Inflation 2/ & & Staples & Other Food & $\begin{array}{l}\text { Drinks \& } \\
\text { Tobacco }\end{array}$ & $\begin{array}{l}\text { Clothes, } \\
\text { Footwear }\end{array}$ & $\begin{array}{r}\text { Household } \\
\text { Goods }\end{array}$ & $\begin{array}{r}\text { Housing, } \\
\text { Construction }\end{array}$ & $\begin{array}{r}\text { Transport, } \\
\text { Post, } \\
\text { Telecom. }\end{array}$ \\
\hline & & 100.0 & 13.1 & 29.6 & 4.5 & 7.6 & 9.2 & 8.2 & 10.1 \\
\hline 2001 January & -0.7 & 100.5 & 102.8 & 100.1 & 100.1 & 100.4 & 99.8 & 99.8 & 101.6 \\
\hline February & -1.8 & 101.0 & 101.7 & 101.4 & 100.4 & 100.0 & 99.9 & 99.8 & 101.5 \\
\hline March & -1.5 & 100.2 & 100.4 & 99.7 & 100.6 & 99.8 & 99.7 & 100.1 & 101.2 \\
\hline April & -1.3 & 99.7 & 98.5 & 99.4 & 100.1 & 100.0 & 99.6 & 100.1 & 100.7 \\
\hline May & -0.9 & 99.5 & 97.3 & 99.7 & 100.4 & 100.0 & 99.7 & 100.0 & 100.4 \\
\hline June & -0.4 & 99.5 & 96.5 & 100.1 & 100.5 & 99.8 & 100.1 & 100.0 & 100.2 \\
\hline July & 0.1 & 99.4 & 95.9 & 99.8 & 99.6 & 100.0 & 100.1 & 100.0 & 100.5 \\
\hline August & 0.0 & 99.4 & 97.0 & 100.1 & 99.4 & 99.4 & 100.2 & 99.6 & 100.4 \\
\hline September & 0.7 & 99.8 & 99.3 & 100.1 & 99.7 & 100.1 & 100.3 & 99.8 & 100.4 \\
\hline October & 0.6 & 99.8 & 99.7 & 99.7 & 99.7 & 100.0 & 100.2 & 100.0 & 99.4 \\
\hline November & -0.1 & 100.0 & 102.5 & 99.7 & 99.8 & 100.1 & 100.2 & 100.4 & 97.0 \\
\hline December & 0.7 & 101.0 & 108.5 & 100.2 & 99.7 & 100.2 & 100.2 & 100.4 & 96.7 \\
\hline Average & -0.4 & 100.0 & 100.0 & 100.0 & 100.0 & 100.0 & 100.0 & 100.0 & 100.0 \\
\hline 2002 January & 1.6 & 102.1 & 112.8 & 101.3 & 100.1 & 100.8 & 100.6 & 100.4 & 96.8 \\
\hline February & 3.4 & 104.4 & 115.9 & 106.0 & 101.5 & 101.3 & 100.9 & 100.6 & 97.8 \\
\hline March & 3.3 & 103.6 & 114.6 & 105.2 & 100.7 & 101.0 & 100.6 & 100.4 & 96.9 \\
\hline April & 3.9 & 103.6 & 112.5 & 106.1 & 100.5 & 100.9 & 100.7 & 100.3 & 96.8 \\
\hline May & 4.4 & 103.9 & 112.0 & 107.5 & 100.9 & 100.7 & 100.7 & 100.6 & 96.7 \\
\hline June & 4.5 & 104.0 & 109.2 & 109.0 & 101.0 & 100.7 & 100.7 & 101.1 & 96.7 \\
\hline July & 4.5 & 103.9 & 107.6 & 109.1 & 101.2 & 100.6 & 100.7 & 101.3 & 96.7 \\
\hline August & 4.6 & 104.0 & 107.5 & 109.1 & 101.4 & 100.7 & 100.8 & 101.5 & 96.8 \\
\hline September & 4.3 & 104.2 & 108.2 & 109.2 & 101.7 & 101.1 & 100.9 & 101.9 & 96.8 \\
\hline October & 4.7 & 104.5 & 108.4 & 108.8 & 102.3 & 101.1 & 100.8 & 105.1 & 97.8 \\
\hline November & 4.8 & 104.8 & 109.6 & 108.2 & 102.6 & 101.2 & 100.8 & 107.1 & 98.4 \\
\hline December & 4.0 & 105.1 & 111.3 & 108.0 & 103.3 & 101.3 & 101.0 & 107.6 & 98.4 \\
\hline Average & 4.0 & 104.0 & 110.8 & 107.3 & 101.4 & 101.0 & 100.8 & 102.3 & 97.2 \\
\hline 2003 January & 3.8 & 106.1 & 113.1 & 109.4 & 104.3 & 102.2 & 101.3 & 108.3 & 98.7 \\
\hline February & 3.8 & 108.4 & 114.5 & 114.2 & 106.6 & 102.8 & 101.5 & 108.8 & 100.0 \\
\hline March & 3.9 & 107.7 & 113.4 & 111.5 & 105.9 & 102.6 & 101.7 & 110.9 & 100.7 \\
\hline April & 3.8 & 107.7 & 112.8 & 111.2 & 105.7 & 103.0 & 101.8 & 111.3 & 100.5 \\
\hline May & 3.6 & 107.6 & 111.8 & 111.1 & 105.5 & 103.5 & 102.0 & 110.8 & 100.3 \\
\hline June & 3.2 & 107.3 & 110.0 & 111.0 & 105.5 & 103.5 & 102.1 & 110.3 & 100.2 \\
\hline July & 3.0 & 107.0 & 108.6 & 110.7 & 105.5 & 103.5 & 102.1 & 110.5 & 100.1 \\
\hline August & 2.8 & 106.9 & 108.8 & 110.3 & 105.6 & 103.5 & 101.8 & 110.8 & 100.0 \\
\hline September & 2.7 & 107.0 & 108.8 & 110.0 & 105.7 & 103.7 & 101.8 & 110.9 & 100.0 \\
\hline October & 2.1 & 106.7 & 109.3 & 109.7 & 106.0 & 103.7 & 102.2 & 111.1 & 95.0 \\
\hline November & 2.4 & 107.3 & 112.4 & 110.0 & 106.3 & 104.2 & 102.7 & 111.7 & 94.6 \\
\hline December & 2.9 & 108.2 & 114.6 & 111.0 & 107.0 & 104.7 & 103.1 & 112.3 & 94.7 \\
\hline Average & 3.2 & 107.3 & 111.5 & 110.9 & 105.8 & 103.4 & 102.0 & 110.6 & 98.8 \\
\hline 2004 January & 3.1 & 109.4 & 117.0 & 112.8 & 110.1 & 106.0 & 103.7 & 113.2 & 94.7 \\
\hline February & 3.9 & 112.6 & 118.8 & 120.5 & 110.7 & 106.3 & 104.4 & 114.7 & 95.3 \\
\hline March & 5.4 & 113.5 & 122.1 & 122.0 & 108.9 & 106.8 & 104.1 & 117.1 & 95.2 \\
\hline April & 5.9 & 114.1 & 124.3 & 122.8 & 109.2 & 107.6 & 104.6 & 117.6 & 95.3 \\
\hline May & 7.0 & 115.1 & 127.1 & 125.0 & 109.6 & 107.5 & 104.7 & 117.8 & 95.5 \\
\hline June & 8.1 & 116.1 & 127.8 & 127.2 & 109.6 & 107.6 & 104.8 & 117.6 & 95.5 \\
\hline July & 9.1 & 116.8 & 126.9 & 128.5 & 109.7 & 107.8 & 105.1 & 118.3 & 98.3 \\
\hline August & 9.9 & 117.5 & 127.9 & 129.5 & 110.2 & 108.2 & 105.5 & 119.0 & 98.3 \\
\hline September & 10.1 & 117.8 & 128.3 & 130.2 & 110.2 & 108.7 & 105.9 & 119.5 & 98.5 \\
\hline October & 10.4 & 117.8 & 128.0 & 129.9 & 110.4 & 108.9 & 106.2 & 119.8 & 98.5 \\
\hline November & 10.0 & 118.0 & 128.9 & 129.5 & 110.4 & 109.1 & 106.3 & 120.4 & 99.6 \\
\hline December & 9.7 & 118.6 & 130.2 & 130.2 & 110.7 & 109.8 & 106.8 & 120.9 & 100.0 \\
\hline Average & 7.7 & 115.6 & 125.6 & 125.7 & 110.0 & 107.9 & 105.2 & 118.0 & 97.1 \\
\hline 2005 January & 9.7 & 119.9 & 132.0 & 132.7 & 111.6 & 110.6 & 107.7 & 121.3 & 100.3 \\
\hline February & 9.1 & 122.9 & 135.3 & 138.4 & 113.4 & 111.1 & 108.1 & 121.8 & 101.1 \\
\hline March & 8.3 & 123.0 & 136.7 & 138.4 & 113.2 & 110.8 & 108.3 & 122.2 & 100.9 \\
\hline April & 8.4 & 123.7 & 137.4 & 139.2 & 113.4 & 111.1 & 108.7 & 123.6 & 102.4 \\
\hline May & 8.0 & 124.4 & 137.7 & 140.3 & 114.4 & 111.5 & 109.0 & 124.6 & 102.8 \\
\hline June & 7.6 & 124.8 & 136.9 & 141.5 & 114.2 & 111.8 & 109.4 & 124.6 & 102.9 \\
\hline July & 7.4 & 125.4 & 136.1 & 141.8 & 114.4 & 112.1 & 109.7 & 126.7 & 105.0 \\
\hline August & 7.2 & 125.9 & 136.1 & 142.3 & 114.8 & 112.8 & 110.3 & 127.4 & 106.0 \\
\hline September & 7.7 & 126.9 & 136.5 & 142.9 & 114.8 & 113.5 & 110.6 & 129.6 & 110.1 \\
\hline October & 8.2 & 127.5 & 137.3 & 143.4 & 115.3 & 114.0 & 111.1 & 131.0 & 110.4 \\
\hline November & 8.5 & 128.0 & 138.8 & 143.5 & 115.6 & 114.5 & 111.4 & 131.9 & 110.6 \\
\hline December 3/ & 8.8 & 129.1 & 140.5 & 145.9 & 116.1 & 115.2 & 112.0 & 132.7 & 109.4 \\
\hline Average & 8.3 & 125.1 & 136.8 & 140.9 & 114.3 & 112.4 & 109.7 & 126.5 & 105.2 \\
\hline 2006 January & 8.9 & 130.6 & 143.5 & 148.0 & 117.5 & 116.3 & 112.6 & 133.8 & 109.5 \\
\hline February & 8.5 & 133.4 & 146.0 & 153.6 & 119.2 & 117.3 & 113.3 & 134.8 & 110.4 \\
\hline March & 7.9 & 132.7 & 146.2 & 151.8 & 118.7 & 117.6 & 113.7 & 135.0 & 109.7 \\
\hline April & 7.4 & 132.9 & 146.6 & 152.1 & 118.7 & 117.8 & 114.2 & 134.6 & 109.8 \\
\hline May & 7.5 & 133.8 & 147.1 & 152.2 & 119.2 & 118.3 & 114.8 & 135.8 & 113.0 \\
\hline June & 7.6 & 134.3 & 147.4 & 152.8 & 119.5 & 118.8 & 115.6 & 137.2 & 113.1 \\
\hline July & 7.5 & 134.8 & 148.0 & 153.6 & 120.3 & 119.1 & 116.0 & 138.2 & 113.3 \\
\hline Average & 7.9 & 133.2 & 146.4 & 152.0 & 119.0 & 117.9 & 114.3 & 135.6 & 111.3 \\
\hline
\end{tabular}

Sources: General Statistics Office (GSO); and Fund staff estimates.

1/ Beginning in May 2006, the weights and composition of the basket were changed. Earlier data have been re-weighted based on the new CPI weights. 2/ Year-on-year percentage change.

3/ Staff estimate of overall rate of inflation as of December 2005 (8.8 percent) differs from GSO estimate (8.4 percent) owing to GSO's utilization of rounding function. 
Table 5. Vietnam: Gross Value of Agricultural Production at Constant Prices, 2001-05

\begin{tabular}{|c|c|c|c|c|c|}
\hline & 2001 & 2002 & 2003 & 2004 & $\frac{2005}{\text { Est. }}$ \\
\hline & \multicolumn{5}{|c|}{ (In billions of dong, at constant 1994 prices) } \\
\hline Total & 112,189 & 119,260 & 124,693 & 129,861 & 134,008 \\
\hline Food crops & 69,798 & 75,814 & 77,611 & 80,810 & 82,225 \\
\hline Paddy and other cereals & 55,066 & 59,619 & 61,029 & 63,621 & 63,690 \\
\hline Vegetables and beans & 6,844 & 7,771 & 8,030 & 8,284 & 8,937 \\
\hline Fruits & 6,402 & 6,895 & 7,017 & 7,355 & 8,008 \\
\hline Other & 1,486 & 1,529 & 1,535 & 1,551 & 1,590 \\
\hline Industrial crops & 23,109 & 22,247 & 24,175 & 25,612 & 25,615 \\
\hline \multirow[t]{2}{*}{ Animal husbandry } & 19,282 & 21,200 & 22,907 & 23,439 & 26,168 \\
\hline & \multicolumn{5}{|c|}{ (In percent of total production) } \\
\hline Food crops & 62.2 & 63.6 & 62.2 & 62.2 & 61.4 \\
\hline Paddy and other cereals & 49.1 & 50.0 & 48.9 & 49.0 & 47.5 \\
\hline Vegetables and beans & 6.1 & 6.5 & 6.4 & 6.4 & 6.7 \\
\hline Fruits & 5.7 & 5.8 & 5.6 & 5.7 & 6.0 \\
\hline Other & 1.3 & 1.3 & 1.2 & 1.2 & 1.2 \\
\hline Industrial crops & 20.6 & 18.7 & 19.4 & 19.7 & 19.1 \\
\hline \multirow[t]{2}{*}{ Animal husbandry } & 17.2 & 17.8 & 18.4 & 18.0 & 19.5 \\
\hline & \multicolumn{5}{|c|}{ (Annual percentage change) } \\
\hline Total & 2.6 & 6.3 & 4.6 & 4.1 & 3.2 \\
\hline Food crops & 1.0 & 8.6 & 2.4 & 4.1 & 1.8 \\
\hline Paddy and other cereals & -0.2 & 8.3 & 2.4 & 4.2 & 0.1 \\
\hline Vegetables and beans & 8.1 & 13.5 & 3.3 & 3.2 & 7.9 \\
\hline Fruits & 4.8 & 7.7 & 1.8 & 4.8 & 8.9 \\
\hline Other & 0.7 & 2.9 & 0.4 & 1.0 & 2.5 \\
\hline Industrial crops & 6.1 & -3.7 & 8.7 & 5.9 & 0.0 \\
\hline Animal husbandry & 4.2 & 9.9 & 8.1 & 2.3 & 11.6 \\
\hline
\end{tabular}

Source: General Statistics Office. 
Table 6. Vietnam: Production of Food Staples, 2001-05

\begin{tabular}{|c|c|c|c|c|c|}
\hline & 2001 & 2002 & 2003 & 2004 & $\frac{2005}{\text { Est. }}$ \\
\hline & \multicolumn{5}{|c|}{ (In thousands of tons) } \\
\hline \multirow{2}{*}{\multicolumn{6}{|c|}{ By product }} \\
\hline & & & & & \\
\hline Rice paddy & 32,108 & 34,447 & 34,569 & 36,149 & 35,791 \\
\hline Spring crop & 15,474 & 16,720 & 16,823 & 17,078 & 17,332 \\
\hline Autumn crop & 8,328 & 9,189 & 9,401 & 10,431 & 10,415 \\
\hline Winter crop & 8,306 & 8,539 & 8,345 & 8,640 & 8,044 \\
\hline Other staples (gross output) & 7,325 & 8,653 & 10,022 & 10,764 & 11,863 \\
\hline Maize & 2,162 & 2,511 & 3,136 & 3,431 & 3,756 \\
\hline Sweet potato & 1,654 & 1,704 & 1,577 & 1,512 & 1,461 \\
\hline Cassava & 3,509 & 4,438 & 5,309 & 5,821 & 6,646 \\
\hline \multicolumn{6}{|l|}{ By region } \\
\hline North & 13,277 & 14,082 & 14,234 & 15,000 & 14,359 \\
\hline \multirow[t]{2}{*}{ South } & 20,996 & 22,879 & 23,473 & 24,581 & 25,190 \\
\hline & \multicolumn{5}{|c|}{ (Annual percentage change) } \\
\hline Total production & -0.8 & 7.8 & 2.0 & 5.0 & -0.1 \\
\hline Rice paddy & -1.3 & 7.3 & 0.4 & 4.6 & -1.0 \\
\hline Spring crop & -0.6 & 8.0 & 0.6 & 1.5 & 1.5 \\
\hline Autumn crop & -3.4 & 10.3 & 2.3 & 11.0 & -0.2 \\
\hline Winter crop & -0.3 & 2.8 & -2.3 & 3.5 & -6.9 \\
\hline Other staples & 30.7 & 18.1 & 15.8 & 7.4 & 10.2 \\
\hline Maize & 7.8 & 16.2 & 24.9 & 9.4 & 9.5 \\
\hline Sweet potato & 2.7 & 3.0 & -7.4 & -4.1 & -3.4 \\
\hline \multirow[t]{2}{*}{ Cassava } & 76.7 & 26.5 & 19.6 & 9.6 & 14.2 \\
\hline & \multicolumn{5}{|c|}{ (In thousands of hectares) } \\
\hline Total area cultivated & 8,225 & 8,323 & 8,367 & 8,438 & 8,371 \\
\hline \multicolumn{6}{|l|}{ By product } \\
\hline Rice paddy & 7,493 & 7,504 & 7,452 & 7,445 & 7,326 \\
\hline Spring crop & 3,057 & 3,033 & 3,023 & 2,979 & 2,942 \\
\hline Autumn crop & 2,211 & 2,294 & 2,320 & 2,366 & 2,349 \\
\hline Winter crop & 2,225 & 2,178 & 2,109 & 2,101 & 2,036 \\
\hline Other staples & 1,267 & 1,391 & 1,505 & 1,582 & 1,655 \\
\hline Maize & 730 & 816 & 913 & 991 & 1,043 \\
\hline Sweet potato & 245 & 238 & 220 & 202 & 188 \\
\hline Cassava & 292 & 337 & 372 & 389 & 424 \\
\hline \multicolumn{6}{|l|}{ By region } \\
\hline North & 3,052 & 3,077 & 3,110 & 3,136 & 3,131 \\
\hline South & 5,173 & 5,245 & 5,256 & 5,302 & 5,241 \\
\hline Yield & \multicolumn{5}{|c|}{ (In metric tons per hectare) } \\
\hline \multicolumn{6}{|l|}{ By product } \\
\hline Rice paddy & 4.3 & 4.6 & 4.6 & 4.9 & 4.9 \\
\hline Spring crop & 5.1 & 5.5 & 5.6 & 5.7 & 5.9 \\
\hline Autumn crop & 3.8 & 4.0 & 4.1 & 4.4 & 4.4 \\
\hline Winter crop & 3.7 & 3.9 & 4.0 & 4.1 & 4.0 \\
\hline Other staples & 5.8 & 6.2 & 6.7 & 6.8 & 7.2 \\
\hline Maize & 3.0 & 3.1 & 3.4 & 3.5 & 3.6 \\
\hline Sweet potato & 6.8 & 7.2 & 7.2 & 7.5 & 7.8 \\
\hline Cassava & 12.0 & 13.2 & 14.3 & 15.0 & 15.7 \\
\hline \multicolumn{6}{|l|}{ By region } \\
\hline North & 4.4 & 4.6 & 4.6 & 4.8 & 4.6 \\
\hline South & 4.1 & 4.4 & 4.5 & 4.6 & 4.8 \\
\hline \multicolumn{6}{|l|}{ Per capita production } \\
\hline Total staples (in metric tons per capita) & 0.4 & 0.5 & 0.5 & 0.5 & 0.5 \\
\hline Rice & 0.4 & 0.4 & 0.4 & 0.4 & 0.4 \\
\hline Other staples (maize) & 0.0 & 0.0 & 0.0 & 0.0 & 0.0 \\
\hline (in percent of total staples production) & 6.1 & 6.8 & 8.3 & 8.7 & 9.5 \\
\hline Total staples $(1994=100)$ & 138.9 & 149.8 & 152.8 & 160.4 & 160.3 \\
\hline Rice & 136.5 & 146.4 & 146.9 & 153.6 & 152.1 \\
\hline Other staples (maize) & 189.0 & 219.5 & 274.2 & 299.9 & 328.4 \\
\hline Total staples (annual percentage change) & -0.8 & 7.8 & 2.0 & 5.0 & -0.1 \\
\hline Rice & -1.3 & 7.3 & 0.4 & 4.6 & -1.0 \\
\hline Other staples (maize) & 7.8 & 16.2 & 24.9 & 9.4 & 9.5 \\
\hline
\end{tabular}

Sources: Ministry of Agriculture; General Statistics Office; and Fund staff estimates. 
Table 7. Vietnam: Industrial Crop Production and Livestock, 2001-05

\begin{tabular}{|c|c|c|c|c|c|}
\hline & 2001 & 2002 & 2003 & 2004 & $\begin{array}{r}2005 \\
\text { Est. }\end{array}$ \\
\hline & \multicolumn{5}{|c|}{ (In thousands of metric tons) } \\
\hline \multicolumn{6}{|l|}{ Production of annual crops } \\
\hline Cotton & 34 & 40 & 35 & 28 & 29 \\
\hline Jute & 15 & 20 & 12 & 13 & 12 \\
\hline Rush & 65 & 88 & 96 & 90 & 81 \\
\hline Sugarcane & 14,657 & 17,120 & 16,855 & 15,649 & 14,731 \\
\hline Peanuts & 363 & 400 & 406 & 469 & 486 \\
\hline Soybeans & 174 & 206 & 220 & 246 & 292 \\
\hline Tobacco & 32 & 33 & 32 & 23 & 26 \\
\hline \multicolumn{6}{|l|}{ Production of perennial crops } \\
\hline Tea & 340 & 424 & 449 & 514 & 534 \\
\hline Coffee & 841 & 700 & 794 & 836 & 768 \\
\hline Rubber & 313 & 298 & 364 & 419 & 469 \\
\hline Coconut & 892 & 915 & 893 & 960 & 972 \\
\hline \multirow[t]{2}{*}{ Pepper } & 44 & 47 & 69 & 73 & 77 \\
\hline & \multicolumn{5}{|c|}{ (Annual percentage change) } \\
\hline Cotton & 78.7 & 19.0 & -12.2 & -20.2 & 3.2 \\
\hline Jute & 29.2 & 39.7 & -39.2 & 1.6 & -7.1 \\
\hline Rush & 5.0 & 36.6 & 8.7 & -6.3 & -10.4 \\
\hline Sugarcane & -2.6 & 16.8 & -1.5 & -7.2 & -5.9 \\
\hline Peanuts & 2.2 & 10.3 & 1.4 & 15.5 & 3.5 \\
\hline Soybeans & 16.3 & 18.4 & 6.9 & 11.9 & 18.5 \\
\hline Tobacco & 18.1 & 3.8 & -4.2 & -26.4 & 10.7 \\
\hline Tea & 8.1 & 24.7 & 5.9 & 14.5 & 4.0 \\
\hline Coffee & 4.7 & -16.8 & 13.5 & 5.3 & -8.2 \\
\hline \multirow[t]{2}{*}{ Rubber } & 7.5 & -4.6 & 22.1 & 15.3 & 11.8 \\
\hline & \multicolumn{5}{|c|}{ (In thousands of hectares) } \\
\hline Area cultivated of annual crops & 786 & 846 & 835 & 857 & 860 \\
\hline \multicolumn{6}{|l|}{ Of which: } \\
\hline Cotton & 28 & 34 & 28 & 28 & 23 \\
\hline Jute & 8 & 10 & 5 & 5 & 5 \\
\hline Rush & 10 & 12 & 14 & 13 & 12 \\
\hline \multicolumn{6}{|l|}{ Mulberry } \\
\hline Sugarcane & 291 & 320 & 313 & 286 & 266 \\
\hline Peanuts & 245 & 247 & 244 & 264 & 270 \\
\hline Soybeans & 140 & 159 & 166 & 184 & 204 \\
\hline Tobacco & 24 & 27 & 23 & 16 & 17 \\
\hline Area cultivated of perennial crops & 1,476 & 1,492 & 1,511 & 1,554 & 1,593 \\
\hline \multicolumn{6}{|l|}{ Of which: } \\
\hline Tea & 98 & 109 & 116 & 121 & 118 \\
\hline Coffee & 565 & 522 & 510 & 497 & 491 \\
\hline Rubber & 416 & 429 & 441 & 454 & 480 \\
\hline Coconut & 156 & 140 & 134 & 133 & 132 \\
\hline \multirow[t]{2}{*}{ Pepper } & 36 & 48 & 51 & 51.0 & 49 \\
\hline & \multicolumn{5}{|c|}{ (In output per hectare, in metric tons) } \\
\hline Cotton & 1.2 & 1.2 & 1.3 & 1.0 & 1.3 \\
\hline Jute & 1.9 & 2.1 & 2.5 & 2.6 & 2.2 \\
\hline Rush & 6.6 & 7.2 & 6.9 & 6.9 & 6.6 \\
\hline Sugarcane & 50.4 & 53.5 & 53.8 & 54.7 & 55.3 \\
\hline Peanut & 1.5 & 1.6 & 1.7 & 1.8 & 1.8 \\
\hline Soybeans & 1.2 & 1.3 & 1.3 & 1.3 & 1.4 \\
\hline Tobacco & 1.3 & 1.2 & 1.4 & 1.4 & 1.6 \\
\hline Tea & 3.5 & 3.9 & 3.9 & 4.3 & 4.5 \\
\hline Coffee & 1.5 & 1.3 & 1.6 & 1.7 & 1.6 \\
\hline \multirow[t]{2}{*}{ Rubber } & 0.8 & 0.7 & 0.8 & 0.9 & 1.0 \\
\hline & \multicolumn{5}{|c|}{ (In millions of heads) } \\
\hline \multicolumn{6}{|l|}{ Livestock } \\
\hline Buffalo & 2.8 & 2.8 & 2.8 & 2.9 & 2.9 \\
\hline Cattle & 3.9 & 4.1 & 4.4 & 4.9 & 5.5 \\
\hline Pigs (over two months old) & 21.8 & 23.2 & 24.9 & 26.1 & 27.4 \\
\hline Poultry & 218.1 & 233.3 & 254.6 & 218.2 & 219.9 \\
\hline
\end{tabular}

Sources: Ministry of Agriculture and Forestry; and General Statistics Office. 
Table 8. Vietnam: Gross Value of Industrial Production at Constant Prices, 2001-05 1/

\begin{tabular}{|c|c|c|c|c|c|}
\hline & 2001 & 2002 & 2003 & 2004 & 2005 \\
\hline & & & & & Est. \\
\hline & \multicolumn{5}{|c|}{ (In trillions of dong, at constant 1994 prices) } \\
\hline Mining & 29.1 & 30.3 & 32.8 & 37.5 & 38.0 \\
\hline Coal & 2.7 & 3.2 & 3.7 & 4.8 & 6.0 \\
\hline Oil and gas & 23.8 & 23.8 & 25.1 & 28.4 & 27.1 \\
\hline Metal ores & 0.2 & 0.3 & 0.3 & 0.5 & 0.7 \\
\hline Stone and other mining & 2.4 & 3.0 & 3.6 & 3.8 & 4.2 \\
\hline Manufacturing & 183.5 & 213.6 & 252.9 & 296.3 & 354.0 \\
\hline Food and beverages & 50.4 & 56.1 & 64.6 & 74.7 & 87.3 \\
\hline Cigarettes and tobacco & 6.7 & 7.7 & 9.2 & 10.2 & 10.7 \\
\hline Textiles and garments & 17.5 & 20.5 & 24.7 & 29.4 & 35.2 \\
\hline Textile & 10.6 & 12.3 & 14.2 & 16.6 & 19.6 \\
\hline Garments & 6.9 & 8.2 & 10.5 & 12.8 & 15.5 \\
\hline Leather tanning and processing & 9.5 & 11.1 & 13.5 & 16.0 & 19.1 \\
\hline Wood and paper products & 8.5 & 9.4 & 11.1 & 13.7 & 16.4 \\
\hline Wood products & 3.9 & 4.5 & 5.5 & 6.6 & 7.8 \\
\hline Paper & 4.6 & 4.9 & 5.7 & 7.1 & 8.6 \\
\hline Printing, copying, publishing & 2.5 & 2.9 & 3.5 & 3.8 & 3.9 \\
\hline Chemical and petroleum products & 13.2 & 15.0 & 16.6 & 19.5 & 22.7 \\
\hline Coke and petroleum & 0.3 & 0.3 & 0.3 & 0.4 & 0.5 \\
\hline Chemicals & 12.9 & 14.7 & 16.3 & 19.0 & 22.2 \\
\hline Rubber and plastics & 8.1 & 9.7 & 11.3 & 15.2 & 19.6 \\
\hline Nonmetallic products & 21.6 & 25.9 & 29.9 & 33.5 & 38.0 \\
\hline Metal products & 13.9 & 17.0 & 21.1 & 24.2 & 29.3 \\
\hline Metallic products & 6.8 & 8.5 & 10.4 & 11.2 & 13.6 \\
\hline Products made of metal & 7.1 & 8.5 & 10.6 & 13.0 & 15.6 \\
\hline Machinery and equipment & 4.4 & 4.7 & 6.2 & 7.2 & 8.8 \\
\hline Computer and office equipment & 1.0 & 1.0 & 1.5 & 1.8 & 2.3 \\
\hline Other machinery and equipment & 3.4 & 3.7 & 4.6 & 5.4 & 6.4 \\
\hline Electric and electronic products & 11.0 & 13.2 & 15.2 & 17.7 & 21.8 \\
\hline Radio, TV, telecommunication equipment & 5.4 & 6.2 & 7.2 & 8.0 & 9.4 \\
\hline Medical instruments & 0.5 & 0.5 & 0.6 & 0.7 & 0.8 \\
\hline Other electric and electronic products & 5.2 & 6.5 & 7.5 & 9.1 & 11.5 \\
\hline Vehicles and transport equipment & 11.4 & 14.3 & 18.0 & 20.9 & 27.3 \\
\hline Assembling and repairing of motor vehicles & 4.3 & 5.8 & 8.3 & 8.7 & 10.7 \\
\hline Transport equipment & 7.1 & 8.5 & 9.7 & 12.2 & 16.6 \\
\hline Furniture and other & 4.9 & 6.1 & 8.0 & 10.4 & 13.9 \\
\hline Furniture & 4.8 & 6.1 & 7.8 & 10.2 & 13.6 \\
\hline Other & 0.2 & 0.2 & 0.2 & 0.3 & 0.3 \\
\hline Electricity, gas, and water & 14.8 & 17.0 & 19.4 & 21.9 & 24.9 \\
\hline Electricity and gas & 13.6 & 15.7 & 18.1 & 20.4 & 23.3 \\
\hline Water supply & 1.2 & 1.3 & 1.4 & 1.5 & 1.6 \\
\hline \multirow[t]{2}{*}{ Total } & 227.4 & 260.9 & 305.1 & 355.6 & 416.9 \\
\hline & \multicolumn{5}{|c|}{ (Annual percentage change) } \\
\hline Mining & 6.7 & 4.2 & 8.1 & 14.4 & 1.4 \\
\hline Oil and gas & 4.7 & 0.1 & 5.6 & 13.0 & -4.5 \\
\hline Manufacturing & 16.1 & 16.4 & 18.4 & 17.2 & 19.5 \\
\hline Food and beverages & 15.4 & 11.3 & 15.2 & 15.7 & 16.9 \\
\hline Textiles and garments & 8.8 & 17.0 & 20.5 & 19.2 & 19.5 \\
\hline Metal products & 19.0 & 22.5 & 23.8 & 14.8 & 21.0 \\
\hline Electric and electronic products & 30.7 & 19.6 & 15.2 & 16.3 & 23.2 \\
\hline Vehicles and transport equipment & 17.6 & 26.3 & 25.4 & 16.0 & 30.9 \\
\hline Electricity, gas, and water & 14.3 & 15.4 & 14.1 & 12.5 & 14.1 \\
\hline Total & 14.7 & 14.7 & 16.9 & 16.6 & 17.2 \\
\hline
\end{tabular}

Source: General Statistics Office.

$1 /$ Due to reclassification of industrial activities, previously published industrial sector data are not comparable with the data in this table. 
Table 9. Vietnam: Industrial Production by Sector of Ownership at Constant Prices, 2001-05 1/

\begin{tabular}{|c|c|c|c|c|c|}
\hline & 2001 & 2002 & 2003 & 2004 & 2005 \\
\hline & & & & & Est. \\
\hline & \multicolumn{5}{|c|}{ (In billions of dong, at constant 1994 prices) } \\
\hline Total industrial production & 227,342 & 261,092 & 305,080 & 355,624 & 416,863 \\
\hline State sector & 93,434 & 105,119 & 117,637 & 131,655 & 143,070 \\
\hline Central & 62,119 & 69,640 & 80,917 & 92,896 & 105,086 \\
\hline Local & 31,315 & 35,479 & 36,720 & 38,759 & 37,984 \\
\hline Nonstate sector & 53,647 & 63,474 & 78,292 & 95,785 & 118,867 \\
\hline Cooperatives & 1,575 & 1,668 & 1,770 & 1,893 & 2,048 \\
\hline Private and mixed & 27,116 & 34,173 & 46,422 & 60,811 & 80,099 \\
\hline Household & 24,956 & 27,633 & 30,101 & 33,081 & 36,720 \\
\hline Foreign invested sector & 80,261 & 92,499 & 109,152 & 128,184 & 154,926 \\
\hline Of which: oil and gas & 23,766 & 23,817 & 25,132 & 28,403 & 27,112 \\
\hline \multirow[t]{2}{*}{ (excluding oil and gas) } & 56,495 & 68,682 & 84,020 & 99,781 & 127,814 \\
\hline & \multicolumn{5}{|c|}{ (In percent of total industrial production) } \\
\hline State sector & 41.1 & 40.3 & 38.6 & 37.0 & 34.3 \\
\hline Central & 27.3 & 26.7 & 26.5 & 26.1 & 25.2 \\
\hline Local & 13.8 & 13.6 & 12.0 & 10.9 & 9.1 \\
\hline Nonstate sector & 23.6 & 24.3 & 25.7 & 26.9 & 28.5 \\
\hline Cooperatives & 0.7 & 0.6 & 0.6 & 0.5 & 0.5 \\
\hline Private and mixed & 11.9 & 13.1 & 15.2 & 17.1 & 19.2 \\
\hline Household & 11.0 & 10.6 & 9.9 & 9.3 & 8.8 \\
\hline Foreign invested sector & 35.3 & 35.4 & 35.8 & 36.0 & 37.2 \\
\hline \multirow[t]{2}{*}{ (excluding oil and gas) } & 24.9 & 26.3 & 27.5 & 28.1 & 30.7 \\
\hline & \multicolumn{5}{|c|}{ (Annual percentage change) } \\
\hline Total industrial production & 14.6 & 14.8 & 16.8 & 16.6 & 17.2 \\
\hline State sector & 12.7 & 12.5 & 11.9 & 11.9 & 8.7 \\
\hline Central & 13.0 & 12.1 & 16.2 & 14.8 & 13.1 \\
\hline Local & 12.1 & 13.3 & 3.5 & 5.6 & -2.0 \\
\hline Nonstate sector & 21.5 & 18.3 & 23.3 & 22.3 & 24.1 \\
\hline Cooperatives & 18.1 & 5.9 & 6.1 & 7.0 & 8.2 \\
\hline Private and mixed & 39.9 & 26.0 & 35.8 & 31.0 & 31.7 \\
\hline Household & 6.5 & 10.7 & 8.9 & 9.9 & 11.0 \\
\hline Foreign invested sector & 12.6 & 15.2 & 18.0 & 17.4 & 20.9 \\
\hline (excluding oil and gas) & 16.4 & 21.6 & 22.3 & 18.8 & 28.1 \\
\hline
\end{tabular}

Sources: General Statistics Office; and Fund staff estimates.

1/ Due to reclassification of industrial activities, previously published industrial sector data are not comparable with the data in this table. 
Table 10. Vietnam: Population and Employment, 2001-05

\begin{tabular}{|c|c|c|c|c|c|}
\hline & \multirow[t]{2}{*}{2001} & \multirow[t]{2}{*}{2002} & \multirow[t]{2}{*}{2003} & \multirow[t]{2}{*}{2004} & \multirow{2}{*}{$\begin{array}{r}2005 \\
\text { Est. }\end{array}$} \\
\hline & & & & & \\
\hline & \multicolumn{4}{|c|}{ (In thousands of persons, unless otherwise indicated) } & \\
\hline Total population & 78,686 & 79,727 & 80,902 & 82,032 & 83,120 \\
\hline (annual percentage change) & 1.4 & 1.3 & 1.5 & 1.4 & 1.3 \\
\hline Urban & 19,469 & 20,022 & 20,869 & 21,737 & 22,419 \\
\hline (annual percentage change) & 3.7 & 2.8 & 4.2 & 4.2 & 3.1 \\
\hline Rural & 59,217 & 59,705 & 60,033 & 60,295 & 60,701 \\
\hline (annual percentage change) & 0.6 & 0.8 & 0.5 & 0.4 & 0.7 \\
\hline Total employment & 38,563 & 39,508 & 40,574 & 41,586 & 42,709 \\
\hline \multirow[t]{2}{*}{ Unemployment rate $1 /$} & 6.3 & 6.0 & 5.8 & 5.6 & 5.3 \\
\hline & \multicolumn{4}{|c|}{ (In percent of total population) } & \\
\hline Urban 2/ & 24.7 & 25.1 & 25.8 & 26.5 & 27.0 \\
\hline Rural 2/ & 75.3 & 74.9 & 74.2 & 73.5 & 73.0 \\
\hline Female & 49.2 & 49.2 & 49.1 & 49.1 & 49.1 \\
\hline Male & 50.8 & 50.8 & 50.9 & 50.9 & 50.9 \\
\hline Age under 15 & 31.3 & 30.3 & 29.3 & 28.0 & 27.1 \\
\hline Age 15-59 & 60.2 & 61.1 & 61.9 & 63.0 & 63.9 \\
\hline Age 60 and over & 8.5 & 8.7 & 8.8 & 9.0 & 9.0 \\
\hline \multirow[t]{2}{*}{ Total employment } & 49.0 & 49.6 & 50.2 & 50.7 & 51.4 \\
\hline & \multicolumn{4}{|c|}{ (In thousands of persons) } & \\
\hline Total employment & 38,563 & 39,508 & 40,574 & 41,586 & 42,709 \\
\hline Nonstate sector & 34,959 & 35,757 & 36,538 & 37,494 & 38,582 \\
\hline State sector $3 /$ & 3,604 & 3,751 & 4,036 & 4,092 & 4,127 \\
\hline Central & 1,499 & 1,569 & 1,628 & 1,662 & 1,683 \\
\hline \multirow[t]{2}{*}{ Local } & 2,105 & 2,181 & 2,408 & 2,430 & 2,444 \\
\hline & \multicolumn{4}{|c|}{ (In percent of total employment) } & \\
\hline Nonstate sector & 90.7 & 90.5 & 90.1 & 90.2 & 90.3 \\
\hline State sector & 9.3 & 9.5 & 9.9 & 9.8 & 9.7 \\
\hline Central & 3.9 & 4.0 & 4.0 & 4.0 & 3.9 \\
\hline \multirow[t]{2}{*}{ Local } & 5.5 & 5.5 & 5.9 & 5.8 & 5.7 \\
\hline & \multicolumn{4}{|c|}{ (Annual percentage change) } & \\
\hline Total employment & 2.5 & 2.5 & 2.7 & 2.5 & 2.7 \\
\hline Nonstate sector & 2.5 & 2.3 & 2.2 & 2.6 & 2.9 \\
\hline State sector & 2.9 & 4.1 & 7.6 & 1.4 & 0.9 \\
\hline Central & 3.9 & 4.7 & 3.8 & 2.1 & 1.3 \\
\hline Local & 2.2 & 3.6 & 10.4 & 0.9 & 0.6 \\
\hline
\end{tabular}

Sources: General Statistics Office; Ministry of Labour, Invalids, and Social Affairs (MOLISA); and Fund staff estimates.

1/ For labor force in urban area comprising males 15 to 60 years old and females 15 to 55 years old. 2/ Armed forces and some other special groups are excluded from urban/rural categories.

3/ As reported by GSO. 
Table 11. Vietnam: Total and Nonstate Employment by Sector, 2001-05

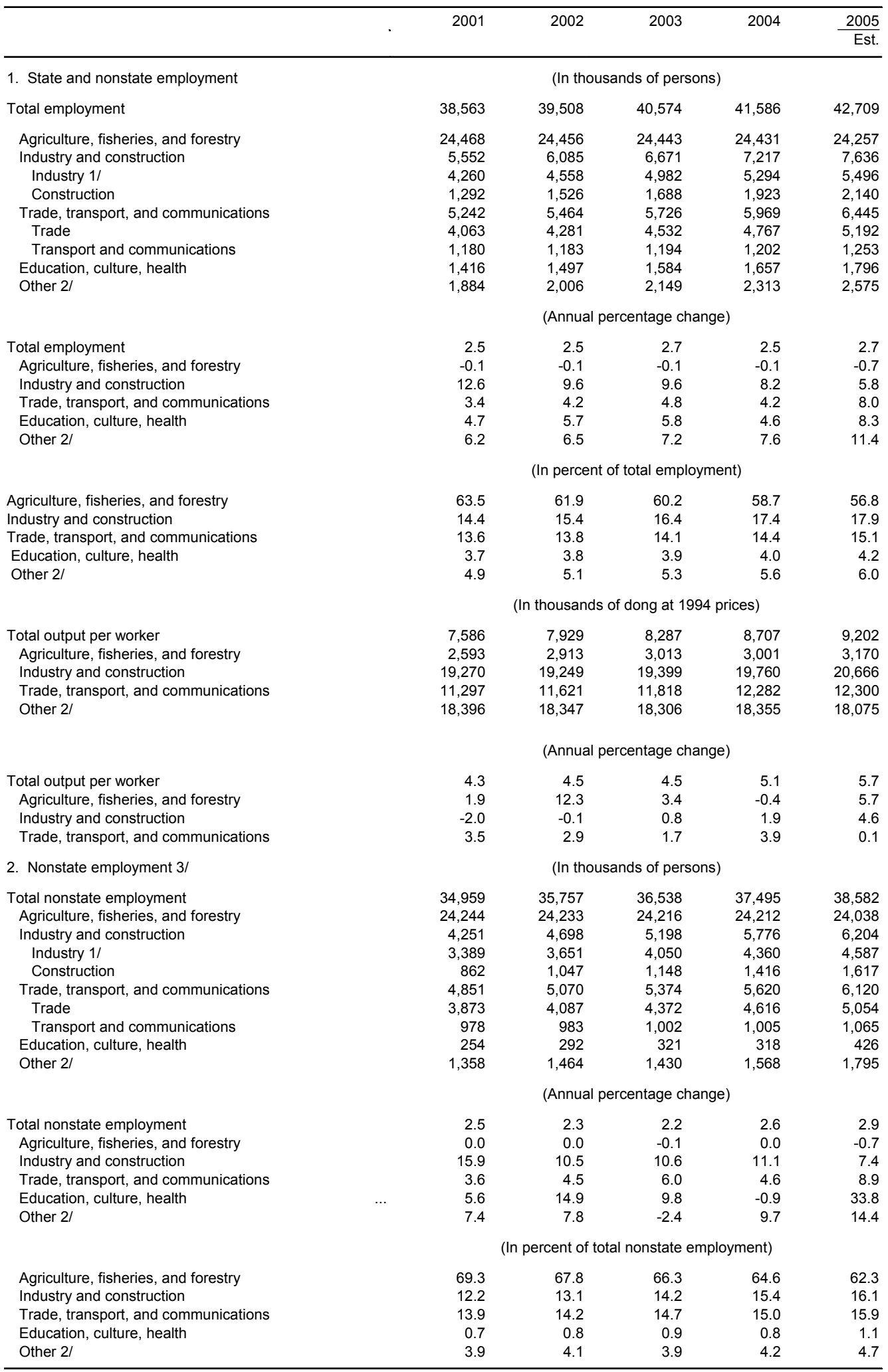

Sources: General Statistics Office; and Ministry of Labor, Invalids and Social Affairs (MOLISA); and Fund staff estimates.

$1 /$ Includes mining and quarrying and electricity, gas, and water supply.

2/ Includes unclassified workers.

3/ Employment outside central and local government and state-owned enterprises. 
Table 12. Vietnam: Employment in the State Sector, 2001-05

\begin{tabular}{|c|c|c|c|c|c|}
\hline & 2001 & 2002 & 2003 & 2004 & $\frac{2005}{\text { Est. }}$ \\
\hline & \multicolumn{5}{|c|}{ (In thousands of persons) } \\
\hline Total employment & 3,604 & 3,751 & 4,035 & 4,108 & 4,127 \\
\hline Agriculture, forestry and fisheries & 225 & 223 & 227 & 221 & 219 \\
\hline Industry and construction & 1,301 & 1,387 & 1,473 & 1,461 & 1,432 \\
\hline Industry & 871 & 908 & 933 & 930 & 909 \\
\hline Construction & 429 & 479 & 540 & 531 & 523 \\
\hline Trade, transport, and communications & 391 & 394 & 353 & 348 & 326 \\
\hline Trade & 190 & 194 & 161 & 151 & 138 \\
\hline Transport and communications & 202 & 200 & 192 & 197 & 188 \\
\hline Education, health, science, and arts & 1,195 & 1,239 & 1,292 & 1,337 & 1,371 \\
\hline Education & 936 & 971 & 1,018 & 1,050 & 1,076 \\
\hline Science & 33 & 34 & 29 & 29 & 29 \\
\hline Culture, arts, and sport & 36 & 37 & 39 & 40 & 43 \\
\hline Public health & 190 & 197 & 206 & 218 & 223 \\
\hline \multirow[t]{2}{*}{ Other } & 492 & 508 & 691 & 741 & 779 \\
\hline & \multicolumn{5}{|c|}{ (In percent of total state sector employment) } \\
\hline Agriculture and forestry & 6.2 & 5.9 & 5.6 & 5.4 & 5.3 \\
\hline Industry and construction & 36.1 & 37.0 & 36.5 & 35.6 & 34.7 \\
\hline Trade, transport, and communications & 10.9 & 10.5 & 8.7 & 8.5 & 7.9 \\
\hline Education, health, science, and arts & 33.2 & 33.0 & 32.0 & 32.5 & 33.2 \\
\hline \multirow{2}{*}{ Other } & 13.7 & 13.5 & 17.1 & 18.0 & 18.9 \\
\hline & \multicolumn{5}{|c|}{ (Annual percentage change) } \\
\hline Total employment & 2.9 & 4.1 & 7.6 & 1.8 & 0.5 \\
\hline Agriculture and forestry & -0.4 & -0.8 & 1.8 & -2.6 & -0.9 \\
\hline Industry and construction & 3.0 & 6.6 & 6.2 & -0.8 & -2.0 \\
\hline Industry & 0.9 & 4.2 & 2.8 & -0.3 & -2.3 \\
\hline Construction & 7.6 & 11.6 & 12.7 & -1.6 & -1.5 \\
\hline Trade, transport, and communications & 0.1 & 0.7 & -10.5 & -1.3 & -6.3 \\
\hline Trade & -0.8 & 2.1 & -17.1 & -5.9 & -8.6 \\
\hline Transport & 1.0 & -0.5 & -4.1 & 2.6 & -4.6 \\
\hline Education, health, science, and arts & 4.3 & 3.7 & 4.3 & 3.4 & 2.2 \\
\hline Science & -1.8 & 1.5 & -14.5 & 0.0 & 0.0 \\
\hline Education & 5.3 & 3.7 & 4.9 & 3.1 & 2.5 \\
\hline Culture, arts, and sport & -0.3 & 3.9 & 3.7 & 1.8 & 7.5 \\
\hline Public health & 1.6 & 3.8 & 4.7 & 5.7 & 2.3 \\
\hline \multirow[t]{2}{*}{ Other } & 3.3 & 3.1 & 36.1 & 7.3 & 5.1 \\
\hline & \multicolumn{5}{|c|}{ (In thousands of dong per month) } \\
\hline Average nominal wage 1 / & 954 & 1,069 & 1,247 & 1,421 & 1,651 \\
\hline Industry & 1548 & 1703 & 1925 & 1,867 & 2,198 \\
\hline Construction & 961 & 1,104 & 1,261 & 1,361 & 1,960 \\
\hline Agriculture, forestry & 590 & 740 & 988 & 1,250 & 1,031 \\
\hline Transportation & 1,667 & 1,910 & 2,278 & 2,433 & 2,345 \\
\hline Trade & 962 & 1,127 & 1,323 & 1,468 & 1,653 \\
\hline Education & 725 & 783 & 1,016 & 1,115 & 1,293 \\
\hline Science & 778 & 895 & 1,051 & 1,165 & 1,597 \\
\hline Culture, arts, and sports & 718 & 815 & 994 & 1,082 & 1,321 \\
\hline Public health & 725 & 797 & 950 & 1,024 & 1,228 \\
\hline State management & 659 & 691 & 797 & 898 & 1,093 \\
\hline \multirow[t]{2}{*}{ Financial intermediation } & 1,804 & 1,935 & 2,321 & 2,791 & 2,746 \\
\hline & & Annual $p$ & centage & lange) & \\
\hline Average nominal wage growth & 12.3 & 12.0 & 16.6 & 14.0 & 16.2 \\
\hline Industry & 17.1 & 10.0 & 13.0 & -3.0 & 17.7 \\
\hline Construction & 11.7 & 14.9 & 14.2 & 7.9 & 44.0 \\
\hline Agriculture & -13.3 & 25.5 & 33.4 & 26.6 & -17.5 \\
\hline Transportation & 9.3 & 14.6 & 19.2 & 6.8 & -3.6 \\
\hline Trade & 8.8 & 17.2 & 17.3 & 11.0 & 12.6 \\
\hline Science & 12.3 & 15.1 & 29.6 & 9.7 & 16.0 \\
\hline Education & 17.9 & 8.0 & 17.3 & 10.8 & 37.1 \\
\hline Culture, arts, and sports & 18.3 & 13.4 & 22.0 & 8.9 & 22.1 \\
\hline Public health & 16.5 & 9.8 & 19.3 & 7.8 & 20.0 \\
\hline State management & 13.5 & 5.0 & 15.3 & 12.7 & 21.7 \\
\hline Financial intermediation & 24.1 & 7.2 & 20.0 & 20.2 & -1.6 \\
\hline Average real wage growth $2 /$ & 12.8 & 7.7 & 13.1 & 5.8 & 7.3 \\
\hline Industry & 17.5 & 5.8 & 9.6 & -10.0 & 8.7 \\
\hline Construction & 12.1 & 10.4 & 10.7 & 0.2 & 33.0 \\
\hline Agriculture & -12.9 & 20.7 & 29.3 & 17.5 & -23.8 \\
\hline Transportation & $\begin{array}{r}-12.9 \\
9.7\end{array}$ & 10.2 & 15.6 & -0.8 & -11.0 \\
\hline Trade & 9.2 & 12.7 & 13.7 & 3.0 & 4.0 \\
\hline Education & 18.4 & 3.9 & 25.6 & 1.9 & 7.1 \\
\hline Science & 12.8 & 10.6 & 13.7 & 2.9 & 26.6 \\
\hline Culture, arts, and sports & 18.8 & 9.1 & 18.3 & 1.1 & 12.7 \\
\hline Public health & 17.0 & 5.6 & 15.6 & 0.1 & 10.8 \\
\hline State management & 13.9 & 0.9 & 11.8 & 4.6 & 12.4 \\
\hline Financial intermediation & 24.5 & 3.1 & 16.3 & 11.6 & -9.2 \\
\hline Consumer price inflation & -0.4 & 4.0 & 3.2 & 7.7 & 8.3 \\
\hline
\end{tabular}

Sources: General Statistics Office; and Fund staff estimates.

1/ Cash income, including payments in kind, bonus payments, and social security contributions. 2/ Nominal wage growth deflated by consumer price inflation. 
Table 13. Vietnam: Summary of General Government Budgetary Operations, 2001-06

\begin{tabular}{|c|c|c|c|c|c|c|}
\hline & 2001 & 2002 & 2003 & 2004 & 2005 & 2006 \\
\hline & & & & & \multicolumn{2}{|c|}{ Prel. Budget 1/ } \\
\hline & \multicolumn{6}{|c|}{ (In trillions of dong) } \\
\hline Revenue and grants $2 /$ & 103.9 & 121.7 & 153.0 & 191.3 & 217.1 & 237.9 \\
\hline Oil revenues $3 /$ & 35.7 & 36.3 & 43.1 & 56.9 & 73.4 & 74.2 \\
\hline Nonoil revenue & 66.2 & 83.1 & 106.9 & 131.6 & 141.4 & 161.2 \\
\hline Tax revenue & 55.6 & 69.4 & 84.4 & 97.6 & 113.4 & $\ldots$ \\
\hline Nontax revenue & 10.6 & 13.7 & 22.5 & 34.0 & 28.0 & $\ldots$ \\
\hline Grants & 2.0 & 2.3 & 3.0 & 2.9 & 2.3 & 2.5 \\
\hline Expenditure & 117.3 & 129.4 & 160.4 & 184.8 & 226.9 & 263.0 \\
\hline Current Expenditure 4/ & 77.0 & 84.2 & 102.5 & 121.2 & 155.0 & 181.4 \\
\hline Wages and salaries $5 /$ & 17.5 & 18.5 & 22.9 & 34.5 & 65.0 & 76.1 \\
\hline Interest payments & 4.5 & 5.3 & 6.4 & 7.2 & 7.0 & 8.8 \\
\hline Other current expenditure & 55.1 & 60.4 & 73.3 & 79.5 & 83.0 & 96.6 \\
\hline Capital Expenditure & 40.2 & 45.2 & 57.8 & 63.6 & 72.0 & 81.6 \\
\hline Official budget balance (authorities' definition) & -13.4 & -7.7 & -7.4 & 6.5 & -9.9 & -25.1 \\
\hline Off-budget investment and net-lending $6 /$ & 10.6 & 17.7 & 32.1 & 26.4 & 39.4 & 45.3 \\
\hline Overall fiscal balance (IMF definition) & -24.0 & -25.4 & -39.5 & -19.8 & -49.3 & -70.4 \\
\hline Financing & 24.0 & 25.4 & 39.5 & 19.8 & 49.3 & 70.4 \\
\hline Domestic (net) & 14.2 & 15.3 & 25.6 & 6.5 & 27.4 & 52.8 \\
\hline \multirow[t]{2}{*}{ Foreign (net) } & 9.8 & 10.2 & 13.8 & 13.3 & 21.8 & 17.7 \\
\hline & \multicolumn{6}{|c|}{ (In percent of GDP) } \\
\hline Revenue and grants $2 /$ & 21.6 & 22.7 & 24.9 & 26.7 & 25.9 & 24.5 \\
\hline Oil revenues $3 /$ & 7.4 & 6.8 & 7.0 & 7.9 & 8.8 & 7.6 \\
\hline Nonoil revenue & 13.8 & 15.5 & 17.4 & 18.4 & 16.9 & 16.6 \\
\hline Tax revenue & 11.6 & 13.0 & 13.8 & 13.6 & 13.5 & $\ldots$ \\
\hline Nontax revenue & 2.2 & 2.6 & 3.7 & 4.8 & 3.3 & $\cdots$ \\
\hline Grants & 0.4 & 0.4 & 0.5 & 0.4 & 0.3 & 0.3 \\
\hline Expenditure & 24.4 & 24.2 & 26.1 & 25.8 & 27.1 & 27.1 \\
\hline Current Expenditure 4/ & 16.0 & 15.7 & 16.7 & 16.9 & 18.5 & 18.7 \\
\hline Wages and salaries 5/ & 3.6 & 3.4 & 3.7 & 4.8 & 7.8 & 7.8 \\
\hline Interest payments & 0.9 & 1.0 & 1.0 & 1.0 & 0.8 & 0.9 \\
\hline Other current expenditure & 11.5 & 11.3 & 11.9 & 11.1 & 9.9 & 10.0 \\
\hline Capital Expenditure & 8.4 & 8.4 & 9.4 & 8.9 & 8.6 & 8.4 \\
\hline Official budget balance (authorities' definition) & -2.8 & -1.4 & -1.2 & 0.9 & -1.2 & -2.6 \\
\hline Off-budget investment and net-lending $6 /$ & 2.2 & 3.3 & 5.2 & 3.7 & 4.7 & 4.7 \\
\hline Overall fiscal balance (IMF definition) & -5.0 & -4.7 & -6.4 & -2.8 & -5.9 & -7.3 \\
\hline Financing & 5.0 & 4.7 & 6.4 & 2.8 & 5.9 & 7.3 \\
\hline Domestic (net) & 2.9 & 2.9 & 4.2 & 0.9 & 3.3 & 5.4 \\
\hline Foreign (net) & 2.0 & 1.9 & 2.3 & 1.9 & 2.6 & 1.8 \\
\hline
\end{tabular}

Sources: Ministry of Finance; and Fund staff estimates.

1/ Budget refers to the 2006 budget approved by the National Assembly, with revenues based on an oil price of US $\$ 56.70$ and the contingency included in expenditure. Off-budget expenditure comprises off-budget investment approved by the Prime Minister and contained in the SEDP; local investment financed via municipal 2/ Staff's adjusted figures from 2003 onward to exclude financing at the local level, which is contained in official 3 / Staff estimates. Includes revenue from crude oil as well as import and domestic taxes related to petroleum products.

4/ Current expenditure includes subsidies to oil traders to cover less-than-complete pass-through of international prices to domestic prices. The 2006 budget likely underestimates subsidies due to the rise in 5 / Staff estimates, as no official data are available.

$6 /$ Includes investment financed through the issuance of infrastructure and education bonds; SOCB recapitalization bonds issued in 2002-04; domestic and ODA-financed on-lending; and sovereign issuance for 
Table 14. Vietnam: Government Revenues, 2001-06

\begin{tabular}{|c|c|c|c|c|c|c|}
\hline & 2001 & 2002 & 2003 & 2004 & $\frac{2005}{\text { Est. }}$ & $\frac{2006}{\text { Budget }}$ \\
\hline & \multicolumn{6}{|c|}{ (In trillions of dong) } \\
\hline Total revenue and grants & 103.9 & 121.7 & 153.0 & 191.3 & 217.1 & 237.9 \\
\hline Tax revenue & 75.9 & 90.2 & 118.5 & 138.2 & 166.2 & 189.9 \\
\hline Corporate income tax & 25.8 & 29.3 & 47.4 & 57.0 & 71.7 & 82.1 \\
\hline Individual income tax & 2.1 & 2.3 & 3.0 & 3.5 & 4.2 & 5.1 \\
\hline Capital user charge & 1.7 & 0.0 & 0.0 & 0.0 & 0.0 & 0.0 \\
\hline Land and housing tax & 0.3 & 0.3 & 0.4 & 0.4 & 0.5 & 0.5 \\
\hline License tax & 0.4 & 0.4 & 0.8 & 0.7 & 0.7 & 0.7 \\
\hline Tax on the transfer of properties & 1.2 & 1.3 & 1.8 & 2.6 & 2.8 & 3.2 \\
\hline Tax on land use right & 0.3 & 0.3 & 0.4 & 0.6 & 1.0 & 0.8 \\
\hline Value added tax (VAT) & 19.3 & 25.9 & 33.1 & 38.8 & 45.7 & 57.1 \\
\hline Excises & 6.2 & 7.3 & 8.9 & 12.8 & 15.7 & 18.2 \\
\hline Slaughter tax & 0.0 & 0.0 & 0.0 & 0.0 & 0.0 & 0.0 \\
\hline Agricultural tax & 0.8 & 0.8 & 0.2 & 0.1 & 0.1 & 0.1 \\
\hline Import and export taxes & 17.5 & 21.9 & 22.4 & 21.6 & 23.6 & 22.0 \\
\hline Other taxes on trade & 0.1 & 0.2 & 0.1 & 0.0 & 0.0 & 0.0 \\
\hline Other taxes & 0.2 & 0.2 & 0.1 & 0.0 & 0.0 & 0.0 \\
\hline Nontax revenue & 26.0 & 29.2 & 31.6 & 50.2 & 48.5 & 45.6 \\
\hline Fees and charges & 5.1 & 6.0 & 14.6 & 22.0 & 21.0 & 21.9 \\
\hline Rental of land & 0.6 & 0.5 & 0.5 & 1.0 & 1.0 & 0.8 \\
\hline Income from natural resources & 8.4 & 8.5 & 9.7 & 17.4 & 21.9 & 18.8 \\
\hline Net profit after tax $1 /$ & 7.5 & 7.6 & 0.0 & 0.0 & 0.0 & 0.0 \\
\hline Capital revenues & 1.0 & 1.1 & 1.1 & 1.3 & 0.9 & 1.1 \\
\hline Other & 3.4 & 5.5 & 5.6 & 8.5 & 3.8 & 2.9 \\
\hline \multirow[t]{2}{*}{ Grants } & 2.0 & 2.3 & 3.0 & 2.9 & 2.3 & 2.5 \\
\hline & \multicolumn{6}{|c|}{ (In percent of GDP) } \\
\hline Total revenue and grants & 21.6 & 22.7 & 24.9 & 26.7 & 25.9 & 24.5 \\
\hline Tax revenue & 15.8 & 16.8 & 19.3 & 19.3 & 19.8 & 19.6 \\
\hline Corporate income tax & 5.4 & 5.5 & 7.7 & 8.0 & 8.6 & 8.5 \\
\hline Individual income tax & 0.4 & 0.4 & 0.5 & 0.5 & 0.5 & 0.5 \\
\hline Capital user charge & 0.4 & 0.0 & 0.0 & 0.0 & 0.0 & 0.0 \\
\hline Land and housing tax & 0.1 & 0.1 & 0.1 & 0.1 & 0.1 & 0.1 \\
\hline License tax & 0.1 & 0.1 & 0.1 & 0.1 & 0.1 & 0.1 \\
\hline Tax on the transfer of properties & 0.2 & 0.2 & 0.3 & 0.4 & 0.3 & 0.3 \\
\hline Tax on land use right & 0.1 & 0.1 & 0.1 & 0.1 & 0.1 & 0.1 \\
\hline VAT & 4.0 & 4.8 & 5.4 & 5.4 & 5.5 & 5.9 \\
\hline Excises & 1.3 & 1.4 & 1.4 & 1.8 & 1.9 & 1.9 \\
\hline Slaughter tax & 0.0 & 0.0 & 0.0 & 0.0 & 0.0 & 0.0 \\
\hline Agricultural tax & 0.2 & 0.1 & 0.0 & 0.0 & 0.0 & 0.0 \\
\hline Import and export taxes & 3.6 & 4.1 & 3.6 & 3.0 & 2.8 & 2.3 \\
\hline Othe taxes on trade & 0.0 & 0.0 & 0.0 & 0.0 & 0.0 & 0.0 \\
\hline Other taxes & 0.0 & 0.0 & 0.0 & 0.0 & 0.0 & 0.0 \\
\hline Nontax revenue & 5.4 & 5.5 & 5.1 & 7.0 & 5.8 & 4.7 \\
\hline Fees and charges & 1.1 & 1.1 & 2.4 & 3.1 & 2.5 & 2.3 \\
\hline Rental of land & 0.1 & 0.1 & 0.1 & 0.1 & 0.1 & 0.1 \\
\hline Income from natural resources & 1.7 & 1.6 & 1.6 & 2.4 & 2.6 & 1.9 \\
\hline Net profit after tax $1 /$ & 1.6 & 1.4 & 0.0 & 0.0 & 0.0 & 0.0 \\
\hline Capital revenues & 0.2 & 0.2 & 0.2 & 0.2 & 0.1 & 0.1 \\
\hline Other & 0.7 & 1.0 & 0.9 & 1.2 & 0.5 & 0.3 \\
\hline Grants & 0.4 & 0.4 & 0.5 & 0.4 & 0.3 & 0.3 \\
\hline
\end{tabular}

Sources: Ministry of Finance; and Fund staff estimates.

1/ Included under corporate income tax starting in 2003. 
Table 15. Vietnam: Government Expenditures, 2001-06

\begin{tabular}{|c|c|c|c|c|c|c|}
\hline & 2001 & 2002 & 2003 & 2004. & $\frac{2005}{\text { Est. }}$ & $\frac{2006}{\text { Budget } 1 /}$ \\
\hline & \multicolumn{6}{|c|}{ (In trillions of dong) } \\
\hline Total expenditure (cash basis, excl. off-budget & 117.3 & 129.4 & 160.4 & 184.8 & 226.9 & 263.0 \\
\hline Total current expenditure & 77.0 & 84.2 & 102.5 & 121.2 & 155.0 & 181.4 \\
\hline General administrative services & 8.7 & 8.6 & 11.4 & 15.9 & 16.8 & 15.0 \\
\hline Economic services & 6.3 & 8.0 & 8.2 & 10.3 & 12.8 & 11.8 \\
\hline Social services & 37.4 & 40.7 & 50.1 & 55.2 & 77.3 & 75.6 \\
\hline Education & 12.0 & 13.8 & 17.4 & 20.4 & 29.1 & 29.5 \\
\hline Health & 4.2 & 4.7 & 5.4 & 6.0 & 10.7 & 10.7 \\
\hline Social subsidies & 13.4 & 13.2 & 16.5 & 17.3 & 23.6 & 21.5 \\
\hline Other & 7.7 & 9.1 & 10.9 & 11.5 & 13.8 & 14.0 \\
\hline Other noninterest expenditure & 20.2 & 21.6 & 26.5 & 32.7 & 41.1 & 70.1 \\
\hline Interest payments (paid) & 4.5 & 5.3 & 6.4 & 7.2 & 7.0 & 8.8 \\
\hline Capital expenditure & 40.2 & 45.2 & 57.8 & 63.6 & 72.0 & 81.6 \\
\hline Off-budget investment expenditure & 10.6 & 17.7 & 32.1 & 26.4 & 39.4 & 45.3 \\
\hline Education bonds & 0.0 & 0.0 & 2.6 & 0.0 & 2.8 & 2.5 \\
\hline Infrastructure bonds & 0.0 & 0.0 & 5.0 & 5.0 & 10.6 & 15.5 \\
\hline Reform bonds (pre-2000 SOCB debt) & 0.0 & 4.9 & 3.2 & 1.5 & 0.0 & 0.0 \\
\hline Municipal bonds & 0.0 & 0.0 & 1.8 & 2.6 & 3.5 & 3.0 \\
\hline \multirow[t]{2}{*}{ On-lending 2/ } & 10.6 & 12.8 & 19.5 & 17.3 & 22.5 & 24.3 \\
\hline & \multicolumn{6}{|c|}{ (In percent of GDP) } \\
\hline Total expenditure (cash basis, excl. off-budget & 24.4 & 24.2 & 26.1 & 25.8 & 27.1 & 27.1 \\
\hline Total current expenditure & 16.0 & 15.7 & 16.7 & 16.9 & 18.5 & 18.7 \\
\hline General administrative services & 1.8 & 1.6 & 1.9 & 2.2 & 2.0 & 1.5 \\
\hline Economic services & 1.3 & 1.5 & 1.3 & 1.4 & 1.5 & 1.2 \\
\hline Social services & 7.8 & 7.6 & 8.2 & 7.7 & 9.2 & 7.8 \\
\hline Education & 2.5 & 2.6 & 2.8 & 2.9 & 3.5 & 3.0 \\
\hline Health & 0.9 & 0.9 & 0.9 & 0.8 & 1.3 & 1.1 \\
\hline Social subsidies & 2.8 & 2.5 & 2.7 & 2.4 & 2.8 & 2.2 \\
\hline Other & 1.6 & 1.7 & 1.8 & 1.6 & 1.7 & 1.4 \\
\hline Other noninterest expenditure & 4.2 & 4.0 & 4.3 & 4.6 & 4.9 & 7.2 \\
\hline Interest payments (paid) & 0.9 & 1.0 & 1.0 & 1.0 & 0.8 & 0.9 \\
\hline Capital expenditure & 8.4 & 8.4 & 9.4 & 8.9 & 8.6 & 8.4 \\
\hline Off-budget investment expenditure & 2.2 & 3.3 & 5.2 & 3.7 & 4.7 & 4.7 \\
\hline Education bonds & 0.0 & 0.0 & 0.4 & 0.0 & 0.3 & 0.3 \\
\hline Infrastructure bonds & 0.0 & 0.0 & 0.8 & 0.7 & 1.3 & 1.6 \\
\hline Reform bonds (pre-2000 SOCB debt) & 0.0 & 0.9 & 0.5 & 0.2 & 0.0 & 0.0 \\
\hline Municipal bonds & 0.0 & 0.0 & 0.3 & 0.4 & 0.4 & 0.3 \\
\hline Onlending $2 /$ & 2.2 & 2.4 & 3.2 & 2.4 & 2.7 & 2.5 \\
\hline
\end{tabular}

Sources: Ministry of Finance; and Fund staff estimates.

1/ Budget comprises budgeted expenditure including the contingency allocation and approved off-budget investment and on-lending.

$2 /$ On-lending includes domestically financed (DAF/VDB), as well as externally financed operations through ODA and sovereign debt issuance. 
Table 16. Vietnam: Treasury Bill and Bond Issues, 2004-06 1/ (In billions of dong, unless otherwise indicated)

\begin{tabular}{|c|c|c|c|c|c|c|c|c|c|}
\hline \multirow{2}{*}{\multicolumn{2}{|c|}{ Auction date }} & \multicolumn{3}{|c|}{ Treasury Bills (one-year) } & \multicolumn{3}{|c|}{ Treasury Bonds (two-year) } & \multicolumn{2}{|c|}{ Treasury Bonds (five-year) } \\
\hline & & $\begin{array}{l}\text { Amount } \\
\text { sold }\end{array}$ & $\begin{array}{l}\text { Average } \\
\text { yield }\end{array}$ & $\begin{array}{c}\text { End-month } \\
\text { outstanding stock }\end{array}$ & $\begin{array}{l}\text { Amount } \\
\text { sold }\end{array}$ & $\begin{array}{c}\text { Average } \\
\text { yield }\end{array}$ & $\begin{array}{c}\text { End-month } \\
\text { outstanding stock }\end{array}$ & $\begin{array}{l}\text { Amount } \\
\text { sold }\end{array}$ & $\begin{array}{c}\text { End-month } \\
\text { outstanding stock }\end{array}$ \\
\hline 2004 & January & 300 & 5.5 & 14,602 & 137 & 8.3 & 12,981 & 1 & 4,497 \\
\hline & February & 1,740 & 5.7 & 15,272 & 441 & 8.3 & 13,224 & 103 & 4,600 \\
\hline & March & 2,600 & 5.7 & 17,052 & 728 & 8.3 & 13,185 & 589 & 5,189 \\
\hline & April & 2,100 & 5.8 & 17,702 & 610 & 8.3 & 13,185 & 1,467 & 6,656 \\
\hline & May & 1,700 & 5.8 & 17,652 & 580 & 8.3 & 13,320 & 757 & 7,413 \\
\hline & June & 2,200 & 5.8 & 19,662 & 411 & 8.3 & 13,464 & 240 & 7,652 \\
\hline & July & 1,472 & 5.9 & 20,234 & 346 & 8.3 & 12,946 & 250 & 7,799 \\
\hline & August & 2,402 & 5.9 & 20,935 & 188 & 8.3 & 12,533 & 525 & 7,735 \\
\hline & September & 1,900 & 5.9 & 19,935 & 154 & 8.3 & 11,906 & 315 & 6,583 \\
\hline & October & 1,400 & 5.9 & 19,435 & 486 & 8.3 & 11,200 & 1,440 & 7,266 \\
\hline & November & 1,250 & 5.6 & 20,013 & 303 & 8.3 & 9,490 & 650 & 7,676 \\
\hline & December & 402 & 6.0 & 19,465 & 220 & 8.3 & 7,877 & 150 & 7,576 \\
\hline 2005 & January & 600 & 6.0 & 19,765 & 163 & 8.4 & 7,939 & 173 & 7,224 \\
\hline & February & 1,000 & 6.0 & 19,025 & 401 & 8.4 & 8,104 & 335 & 7,244 \\
\hline & March & 870 & 6.0 & 17,295 & 409 & 8.4 & 8,273 & 1,340 & 7,144 \\
\hline & April & 350 & 6.1 & 15,545 & 760 & 8.4 & 8,079 & 940 & 7,434 \\
\hline & May & 1,230 & 6.1 & 15,075 & 148 & 8.4 & 8,225 & 2,167 & 9,451 \\
\hline & June & 1,521 & 6.2 & 14,396 & 298 & 8.4 & 8,235 & 1,764 & 11,042 \\
\hline & July & 1,800 & 6.2 & 14,724 & 248 & 8.4 & 8,295 & 598 & 11,305 \\
\hline & August & 1,600 & 6.3 & 13,922 & 211 & 8.4 & 8,347 & 1,936 & 11,901 \\
\hline & September & 4,250 & 6.3 & 16,272 & 251 & 8.4 & 8,314 & 2,129 & 13,090 \\
\hline & October & 6,300 & 6.3 & 21,172 & 339 & 8.4 & 8,278 & 890 & 11,813 \\
\hline & November & 1,900 & 6.3 & 21,822 & 560 & 8.4 & 8,611 & 1,378 & 11,427 \\
\hline & December & 700 & 6.3 & 22,121 & 277 & 8.4 & 8,670 & 1,375 & 12,204 \\
\hline 2006 & January & 500 & 6.3 & 22,021 & 0 & 8.6 & 8,533 & 751 & 11,019 \\
\hline & February & 2,900 & 6.3 & 23,921 & 0 & 8.6 & 8,092 & 1,150 & 10,040 \\
\hline & March & 2,800 & 6.3 & 25,851 & 652 & 8.6 & 8,016 & 4,423 & 13,573 \\
\hline & April & 2,000 & 5.9 & 27,501 & 393 & 8.6 & 7,799 & 2,705 & 14,900 \\
\hline & May & 2,000 & 5.7 & 28,271 & 453 & 8.6 & 7,671 & 2,550 & 16,075 \\
\hline & June & 3,000 & 5.2 & 29,750 & 343 & 8.6 & 7,603 & 2,289 & 17,613 \\
\hline & July & 3,345 & 4.0 & 31,295 & 309 & 8.6 & 7,566 & 780 & 17,243 \\
\hline
\end{tabular}

Sources: State Bank of Vietnam and Ministry of Finance; and Fund staff estimates.

1/ Treasury bills of varying maturities have also been placed directly with the public through the Ministry of Finance's retail network. 
Table 17. Vietnam: Monetary Survey, 2002-06 1/

\begin{tabular}{|c|c|c|c|c|c|c|}
\hline & \multirow[t]{2}{*}{2002} & \multirow[t]{2}{*}{2003} & \multirow[t]{2}{*}{2004} & \multirow{2}{*}{2005} & \multicolumn{2}{|c|}{2006} \\
\hline & & & & & Mar & June \\
\hline Net foreign assets & 117.4 & 131.4 & 145.9 & 191.1 & 218.4 & 227.6 \\
\hline Foreign assets & 135.9 & 150.5 & 172.5 & 220.5 & 247.7 & 257.3 \\
\hline Foreign liabilities & -18.4 & -19.1 & -26.6 & -29.4 & -29.4 & -29.7 \\
\hline Net domestic assets & 211.7 & 279.8 & 386.4 & 499.7 & 521.2 & 555.3 \\
\hline Domestic credit & 239.9 & 316.9 & 434.6 & 585.6 & 591.8 & 621.1 \\
\hline Net claims on government & 8.8 & 20.1 & 14.5 & 32.5 & 30.7 & 23.5 \\
\hline Credit to the economy & 231.1 & 296.7 & 420.0 & 553.1 & 561.1 & 597.6 \\
\hline of which in foreign currency & 46.2 & 65.2 & 104.5 & 134.3 & 134.2 & 141.7 \\
\hline Claims on state enterprises & 89.5 & 105.4 & 142.9 & 181.3 & 182.6 & 192.7 \\
\hline Claims on other sectors & 141.6 & 191.3 & 277.1 & 371.8 & 378.5 & 404.9 \\
\hline Other items, net & -28.2 & -37.0 & -48.1 & -86.0 & -70.6 & -65.9 \\
\hline Total liquidity (M2) & 329.1 & 411.2 & 532.3 & 690.7 & 739.6 & 782.9 \\
\hline of which: total deposits & 254.9 & 320.6 & 423.2 & 559.5 & 602.4 & 649.4 \\
\hline Dong liquidity & 235.5 & 314.1 & 402.7 & 531.5 & 571.3 & 601.0 \\
\hline Currency outside banks & 74.3 & 90.6 & 109.1 & 131.2 & 137.2 & 133.4 \\
\hline Deposits & 161.3 & 223.6 & 293.6 & 400.3 & 434.1 & 467.6 \\
\hline Foreign currency deposits & 93.6 & 97.1 & 129.6 & 159.2 & 168.3 & 181.8 \\
\hline (in millions of U.S. dollars) & 6,093 & 6,220 & 8,235 & 10,027 & 10,575 & 11,395 \\
\hline \multirow[t]{2}{*}{ (in percent of total liquidity) } & 28.4 & 23.6 & 24.3 & 23.0 & 22.8 & 23.2 \\
\hline & \multicolumn{6}{|c|}{ (Annual percentage change, unless otherwise indicated) } \\
\hline Net foreign assets & -0.2 & 11.9 & 11.0 & 39.0 & 44.1 & 51.0 \\
\hline Net domestic assets & 30.6 & 32.2 & 38.1 & 29.3 & 29.5 & 28.9 \\
\hline Domestic credit & 25.5 & 32.1 & 37.1 & 34.7 & 27.5 & 24.8 \\
\hline Credit to the economy & 22.2 & 28.4 & 41.6 & 31.7 & 23.1 & 21.4 \\
\hline of which in foreign currency & 26.6 & 41.0 & 60.3 & 28.5 & 20.1 & 15.6 \\
\hline Claims on state enterprises & 12.2 & 17.8 & 35.6 & 26.9 & 19.6 & 17.9 \\
\hline Claims on other sectors & 29.5 & 35.1 & 44.8 & 34.2 & 24.9 & 23.1 \\
\hline Total liquidity (M2) & 17.6 & 24.9 & 29.5 & 29.7 & 33.5 & 34.6 \\
\hline of which: total deposits & 19.4 & 25.8 & 32.0 & 32.2 & 37.0 & 36.8 \\
\hline Dong liquidity & 23.2 & 33.4 & 28.2 & 32.0 & 35.8 & 36.3 \\
\hline Currency outside banks & 12.0 & 22.0 & 20.4 & 20.2 & 20.1 & 25.0 \\
\hline Deposits & 29.2 & 38.6 & 31.3 & 36.3 & 41.7 & 39.9 \\
\hline \multirow{2}{*}{$\begin{array}{l}\text { Foreign currency deposits } \\
\text { (change during year, in millions of U.S. dollars) }\end{array}$} & 5.6 & 3.7 & 33.5 & 22.8 & 26.2 & 29.3 \\
\hline & \multicolumn{6}{|c|}{ (In percent, unless otherwise indicated) } \\
\hline Velocity $2 /$ & 1.6 & 1.4 & 1.3 & 1.2 & 1.3 & 1.2 \\
\hline Dong velocity $2 /$ & 2.2 & 1.8 & 1.8 & 1.6 & 1.7 & 1.6 \\
\hline Money multiplier $3 /$ & 3.4 & 3.4 & 3.8 & 4.0 & 4.0 & 4.4 \\
\hline Currency/broad money (in percent) & 22.6 & 22.0 & 20.5 & 19.0 & 24.8 & 22.9 \\
\hline Currency/dong deposits (in percent) & 46.1 & 40.5 & 37.2 & 32.8 & 44.8 & 39.9 \\
\hline Currency/total deposits (in percent) & 29.1 & 28.2 & 25.8 & 23.4 & 22.8 & 20.5 \\
\hline Foreign currency deposits/total deposits & 36.7 & 30.3 & 30.6 & 28.5 & 27.9 & 28.0 \\
\hline \multirow[t]{2}{*}{ Foreign currency loans/total loans } & 20.0 & 22.0 & 24.9 & 24.3 & 23.9 & 23.7 \\
\hline & \multicolumn{6}{|c|}{ (In millions of U.S. dollar, unless indicated otherwise) } \\
\hline Gross official international reserves (adjusted) 4/ & 3,692 & 5,620 & 6,314 & 8,557 & 10,185 & 10,925 \\
\hline (in weeks of next year's imports of GNFS) & 7.2 & 8.7 & 8.5 & 9.8 & $\ldots$ & $\ldots$ \\
\hline (change during year, in millions of U.S. dollars) & 305 & 1,929 & 694 & 2,243 & 1,628 & 2,368 \\
\hline Net foreign assets of the banking system & 7,640 & 8,419 & 9,271 & 12,036 & 13,726 & 14,262 \\
\hline (change during year, in millions of U.S. dollars) & -164 & 778 & 852 & 2,766 & 1,690 & 2,226 \\
\hline
\end{tabular}

Sources: State Bank of Vietnam (SBV); and Fund staff estimates.

1/ Data comprise the SBV, six state-owned commercial banks, and 79 non-state credit institutions as of end- June 2006.

2/ Velocity is measured as the ratio of GDP to end-of-period total liquidity (M2) or dong liquidity, respectively.

3 / Money multiplier is measured as the ratio of total liquidity (M2) to reserve money.

4/ Excludes foreign-currency counterpart of swap operations, government foreign-currency deposits at the SBV, and foreign-currency transactions related to the possible issuance of international bonds by the government. 
Table 18. Vietnam: Balance Sheet of State Bank of Vietnam, 2002-06 (In trillions of dong, unless otherwise indicated)

\begin{tabular}{|c|c|c|c|c|c|c|}
\hline & \multirow[t]{2}{*}{2002} & \multirow[t]{2}{*}{2003} & \multirow[t]{2}{*}{2004} & \multirow{2}{*}{$\begin{array}{r}2005 \\
\text { Dec }\end{array}$} & \multicolumn{2}{|c|}{2006} \\
\hline & & & & & Mar & Jun \\
\hline Net foreign assets of SBV & 56.6 & 91.0 & 105.7 & 140.4 & 167.6 & 177.1 \\
\hline Foreign assets & 65.2 & 99.5 & 113.4 & 146.7 & 173.7 & 183.3 \\
\hline Foreign liabilities & -8.6 & -8.4 & -7.7 & -6.2 & -6.2 & -6.1 \\
\hline Net domestic assets & 38.9 & 30.6 & 35.5 & 34.1 & 15.9 & 1.7 \\
\hline Domestic credit (net) & 30.3 & 24.2 & 25.7 & 18.9 & 5.8 & -5.2 \\
\hline Net claims on government & 11.1 & 10.7 & 11.3 & 7.9 & -4.3 & -14.2 \\
\hline Claims on banks & 19.2 & 13.6 & 14.4 & 11.0 & 10.1 & 9.0 \\
\hline Claims on enterprises & 0.0 & 0.0 & 0.0 & 0.0 & 0.0 & 0.0 \\
\hline Claims on nonbank financial institutions & 0.0 & 0.0 & 0.0 & 0.0 & 0.0 & 0.0 \\
\hline Other items net & 8.6 & 6.4 & 9.8 & 15.2 & 10.1 & 6.9 \\
\hline Reserve money & 95.5 & 121.6 & 141.2 & 174.6 & 183.5 & 178.8 \\
\hline Currency in circulation & 77.3 & 94.9 & 114.1 & 137.4 & 144.3 & 141.3 \\
\hline Bankers' deposits & 18.2 & 26.8 & 27.0 & 37.1 & 39.2 & 37.5 \\
\hline \multicolumn{7}{|l|}{ Memorandum items: } \\
\hline Reserve money growth (annual percentage change) & 12.4 & 27.4 & 16.1 & 23.7 & 29.6 & 30.9 \\
\hline Exchange rate (dong/US $\$$, end of period) $1 /$ & 15,368 & 15,608 & 15,739 & 15,875 & 15,910 & 15,957 \\
\hline
\end{tabular}

Sources: State Bank of Vietnam; and Fund staff estimates.

$1 /$ Interbank market rate. 
Table19. Vietnam: Consolidated Balance Sheet of Deposit Money Banks (DMBs), 2002-06 1/ (In trillions of dong, unless otherwise indicated)

\begin{tabular}{|c|c|c|c|c|c|c|}
\hline & \multirow[t]{2}{*}{2002} & \multirow[t]{2}{*}{2003} & \multirow[t]{2}{*}{2004} & \multirow{2}{*}{$\frac{2005}{\text { Dec. }}$} & \multicolumn{2}{|c|}{2006} \\
\hline & & & & & Mar & Jun \\
\hline Net foreign assets of DMBs & 60.8 & 40.4 & 40.2 & 50.6 & 50.8 & 50.5 \\
\hline Foreign assets & 70.7 & 51.1 & 59.1 & 73.8 & 74.0 & 74.0 \\
\hline Foreign liabilities & -9.9 & -10.7 & -18.9 & -23.2 & -23.2 & -23.5 \\
\hline Net domestic assets of DMBs & 194.1 & 280.3 & 383.0 & 508.9 & 551.6 & 599.0 \\
\hline Domestic credit (net) from the DMBs & 228.8 & 306.2 & 423.3 & 577.7 & 596.1 & 635.3 \\
\hline Net claims on government by DMBs & -2.3 & 9.5 & 3.2 & 24.6 & 35.0 & 37.7 \\
\hline Credit to the economy by DMBs & 231.1 & 296.7 & 420.0 & 553.1 & 561.1 & 597.6 \\
\hline Claims on enterprises & 89.5 & 105.4 & 142.9 & 181.3 & 182.6 & 192.7 \\
\hline Claims on other sectors & 141.6 & 191.3 & 277.1 & 371.8 & 378.5 & 404.9 \\
\hline Other domestic assets (net) & -34.7 & -25.9 & -40.3 & -68.8 & -44.6 & -36.4 \\
\hline Reserves & 20.2 & 30.8 & 31.6 & 43.0 & 45.7 & 43.3 \\
\hline Credit from SBV & -19.3 & -14.5 & -16.7 & -20.9 & -10.5 & -10.6 \\
\hline Capital and reserves & -30.9 & -41.7 & -57.6 & -65.0 & -71.6 & -75.0 \\
\hline Other items (net) & -4.7 & -0.5 & 2.4 & -25.9 & -8.2 & 6.0 \\
\hline Total deposits & 254.9 & 320.6 & 423.2 & 559.5 & 602.4 & 649.4 \\
\hline Dong deposits & 161.3 & 223.6 & 293.6 & 400.3 & 434.1 & 467.6 \\
\hline Demand deposits & 53.7 & 69.8 & 92.8 & 114.9 & 103.5 & 109.7 \\
\hline Time deposits & 107.5 & 153.8 & 200.8 & 285.4 & 330.6 & 357.9 \\
\hline Foreign currency deposits & 93.6 & 97.1 & 129.6 & 159.2 & 168.3 & 181.8 \\
\hline Demand deposits & 86.5 & 92.5 & 123.2 & 152.6 & 161.0 & 172.0 \\
\hline \multirow[t]{2}{*}{ Import/restricted deposits } & 7.1 & 4.6 & 6.4 & 6.6 & 7.3 & 9.8 \\
\hline & \multicolumn{6}{|c|}{ (Annual percentage change, unless otherwise indicated) } \\
\hline Net foreign assets of DMBs & -12.0 & -33.7 & -0.4 & 25.9 & 63.6 & 65.6 \\
\hline Net domestic assets of DMBs & 34.4 & 44.4 & 36.7 & 32.8 & 35.0 & 34.8 \\
\hline Domestic credit (net) from the DMBs & 24.9 & 33.8 & 38.2 & 36.5 & 28.7 & 26.6 \\
\hline Credit to the economy by DMBs & 22.2 & 28.4 & 41.6 & 31.7 & 23.1 & 21.4 \\
\hline Claims on enterprises & 12.2 & 17.8 & 35.6 & 26.9 & $\ldots$ & $\ldots$ \\
\hline Claims on other sectors & 29.5 & 35.1 & 44.8 & 34.2 & $\ldots$ & $\ldots$ \\
\hline Banks' reserves & 9.4 & 52.6 & 2.8 & 35.9 & 78.6 & 53.0 \\
\hline Credit from SBV & 8.4 & -24.7 & 15.1 & 25.6 & -26.1 & -28.8 \\
\hline Capital and reserves & 27.4 & 34.9 & 38.0 & 12.9 & 25.4 & 23.7 \\
\hline Total deposits & 19.4 & 25.8 & 32.0 & 32.2 & 37.0 & 36.8 \\
\hline Dong deposits & 29.2 & 38.6 & 31.3 & 36.3 & 41.7 & 39.9 \\
\hline Foreign currency deposits & 5.6 & 3.7 & 33.5 & 22.8 & 26.2 & 29.3 \\
\hline
\end{tabular}

Sources: State Bank of Vietnam (SBV); and Fund staff estimates.

1/ Data comprise six state-owned commercial banks, and 79 non-state credit institutions as of end- June 2006. 
Table 20. Vietnam: Distribution of Credit, 2001-06 1/ 2/

\begin{tabular}{|c|c|c|c|c|c|c|}
\hline & 2001 & 2002 & 2003 & 2004 & $\frac{2005}{\text { Dec. }}$ & $\frac{2006}{\text { Mar. }}$ \\
\hline & \multicolumn{6}{|c|}{ (In billions of Dong) } \\
\hline Total credit to the economy $3 /$ & 189,103 & 231,078 & 296,737 & 420,335 & 553,106 & 561,100 \\
\hline To state enterprises & 79,745 & 89,500 & 105,400 & 142,900 & 181,306 & 182,600 \\
\hline To other sectors & 109,358 & 141,578 & 191,337 & 277,435 & 371,800 & 378,500 \\
\hline Credit extended by state-owned commercial banks $4 /$ & 136,000 & 175,489 & 214,800 & 315,335 & 381,406 & 378,000 \\
\hline To state enterprises & 72,900 & 81,600 & 95,700 & 145,800 & 158,006 & 155,600 \\
\hline To other sectors & 63,100 & 93,889 & 119,100 & 169,535 & 223,400 & 222,400 \\
\hline Credit extended by other banks $5 /$ & 53,140 & 55,589 & 81,937 & 105,000 & 171,700 & 183,100 \\
\hline To state enterprises & 6,900 & 7,900 & 9,700 & 16,300 & 23,300 & 27,000 \\
\hline To other sectors & 46,200 & 47,689 & 72,237 & 88,700 & 148,400 & 156,100 \\
\hline Total dong loans $3 /$ & 152,763 & 184,829 & 231,700 & 317,400 & 418,700 & 427,000 \\
\hline To state enterprises & 67,600 & 66,500 & 74,900 & 96,700 & 124,800 & 126,800 \\
\hline To other sectors & 85,163 & 118,329 & 156,800 & 220,700 & 293,900 & 300,200 \\
\hline Credit extended by state-owned commercial banks 4 / & 117,000 & 149,877 & 177,700 & 234,400 & 302,800 & 303,800 \\
\hline To state enterprises & 57,800 & 61,500 & 68,700 & 87100 & 109,900 & 109,700 \\
\hline To other sectors & 59,200 & 88,377 & 109,000 & 147,300 & 192,900 & 194,100 \\
\hline Credit extended by other banks $5 /$ & 35,800 & 34,952 & 54,000 & 83,000 & 115,900 & 123,200 \\
\hline To state enterprises & 4,600 & 5,000 & 6,200 & 9,600 & 14,900 & 17,100 \\
\hline To other sectors & 31,200 & 29,952 & 47,800 & 73,400 & 101,000 & 106,100 \\
\hline Total foreign currency loans 3 / & 36,340 & 46,249 & 65,037 & 102,935 & 134,406 & 134,100 \\
\hline To state enterprises & 17,400 & 23,000 & 30,500 & 46,200 & 55,506 & 55,800 \\
\hline To other sectors & 18,940 & 23,249 & 34,537 & 56,735 & 78,900 & 78,300 \\
\hline Credit extended by state-owned commercial banks $4 /$ & 19,000 & 25,612 & 37,137 & 61,735 & 78,606 & 74,200 \\
\hline To state enterprises & 15,100 & 20,100 & 27,000 & 39,500 & 48,106 & 45,900 \\
\hline To other sectors & 3,900 & 5,512 & 10,137 & 22,235 & 30,500 & 28,300 \\
\hline Credit extended by other banks $5 /$ & 17,340 & 20,637 & 27,900 & 41,200 & 55,800 & 59,900 \\
\hline To state enterprises & 2,300 & 2,900 & 3,500 & 6,700 & 8,400 & 9,900 \\
\hline \multirow[t]{2}{*}{ To other sectors } & 15,040 & 17,737 & 24,400 & 34,500 & 47,400 & 50,000 \\
\hline & \multicolumn{6}{|c|}{ (In percent of total credit to the economy) } \\
\hline Total credit to the economy $3 /$ & 100.0 & 100.0 & 100.0 & 100.0 & 100.0 & 100.0 \\
\hline To state enterprises & 42.2 & 38.7 & 35.5 & 34.0 & 32.8 & 32.5 \\
\hline To other sectors & 57.8 & 61.3 & 64.5 & 66.0 & 67.2 & 67.5 \\
\hline Credit extended by state-owned commercial banks $4 /$ & 71.9 & 75.9 & 72.4 & 75.0 & 69.0 & 67.4 \\
\hline To state enterprises & 38.6 & 35.3 & 32.3 & 34.7 & 28.6 & 27.7 \\
\hline To other sectors & 33.4 & 40.6 & 40.1 & 40.3 & 40.4 & 39.6 \\
\hline Credit extended by other banks $5 /$ & 28.1 & 24.1 & 27.6 & 25.0 & 31.0 & 32.6 \\
\hline To state enterprises & 3.6 & 3.4 & 3.3 & 3.9 & 4.2 & 4.8 \\
\hline To other sectors & 24.4 & 20.6 & 24.3 & 21.1 & 26.8 & 27.8 \\
\hline Total dong loans & 80.8 & 80.0 & 78.1 & 75.5 & 75.7 & 76.1 \\
\hline Credit extended by state-owned commercial banks 4 / & 61.9 & 64.9 & 59.9 & 55.8 & 54.7 & 54.1 \\
\hline Credit extended by other banks $5 /$ & 18.9 & 15.1 & 18.2 & 19.7 & 21.0 & 22.0 \\
\hline Total foreign currency loans & 19.2 & 20.0 & 21.9 & 24.5 & 24.3 & 23.9 \\
\hline Credit extended by state-owned commercial banks 4 / & 10.0 & 11.1 & 12.5 & 14.7 & 14.2 & 13.2 \\
\hline Credit extended by other banks $5 /$ & 9.2 & 8.9 & 9.4 & 9.8 & 10.1 & 10.7 \\
\hline & \multicolumn{6}{|c|}{ (In billions of dong) } \\
\hline \multicolumn{7}{|l|}{ Total credit to the economy by sector } \\
\hline Agriculture, fisheries, and forestry & 50,680 & 65,860 & 87,200 & 124,800 & 163,717 & 164,971 \\
\hline Industry and construction & 72,616 & 92,854 & 115,700 & 165,200 & 219,027 & 221,083 \\
\hline Industry & 46,708 & 58,694 & 74,500 & 104500 & 139,934 & 141,404 \\
\hline Construction & 25,908 & 34,160 & 41,200 & 60700 & 79,093 & 79,680 \\
\hline Trade, transportation, and communications & 45,952 & 51,762 & 68,000 & 97,500 & 129,425 & 133,548 \\
\hline Trade & 36,875 & 39,745 & 51,000 & 74400 & 97,345 & 101,564 \\
\hline Transport, storage, and communications & 9,077 & 12,017 & 17,000 & 23100 & 32,080 & 31,984 \\
\hline Other services & 19,855 & 20,602 & 25,837 & 32800 & 40,929 & 41,523 \\
\hline & \multicolumn{6}{|c|}{ (In percent of total credit to the economy) } \\
\hline Agriculture, fisheries, and forestry & 26.8 & 28.5 & 29.4 & 29.7 & 29.6 & 29.4 \\
\hline Industry and construction & 38.4 & 40.2 & 39.0 & 39.3 & 39.6 & 39.4 \\
\hline Trade, transportation, and communications & 24.3 & 22.4 & 22.9 & 23.2 & 23.4 & 23.8 \\
\hline Other services & 10.5 & 8.9 & 8.7 & 7.8 & 7.4 & 7.4 \\
\hline
\end{tabular}

Sources: State Bank of Vietnam; and Fund staff estimates.

1/ Figures on credit to the economy by sector of ownership are estimated rather than actual data.

2/ Data comprise six state-owned commercial banks and 77 non-state credit institutions as of end- March, 2006.

3/ Excludes net credit to the government.

4/ Includes four large state-owned commercial banks.

5/ Includes two small state-owned commercial banks, joint-stock banks, joint-venture banks, branches of foreign banks, and the Central

People's Credit Fund and since December 2005 includes also 5 finance companies. 


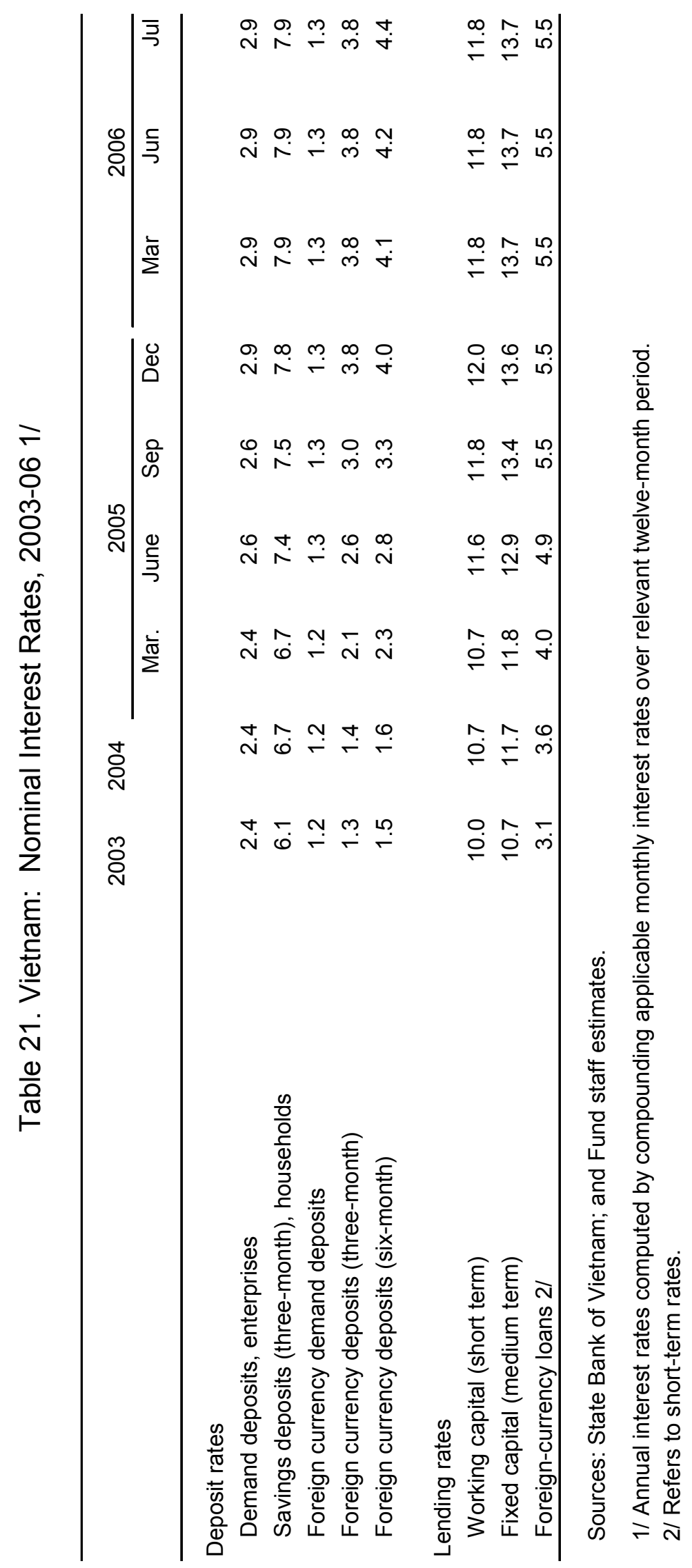


Table 22. Vietnam: Exchange Rate Developments, 2001-06

\begin{tabular}{|c|c|c|c|c|c|c|}
\hline & 2001 & 2002 & 2003 & 2004 & 2005 & $\frac{2006}{\text { Jan-June }}$ \\
\hline & \multicolumn{6}{|c|}{ (period average; $2000=100$ ) } \\
\hline \multicolumn{7}{|l|}{ Effective exchange rates } \\
\hline Nominal & 102.2 & 97.7 & 88.5 & 82.6 & 81.1 & 80.9 \\
\hline \multirow[t]{2}{*}{ Real } & 100.1 & 98.3 & 90.6 & 89.3 & 93.2 & 96.7 \\
\hline & \multicolumn{6}{|c|}{ (Annual percentage change; - depreciation) } \\
\hline Nominal & 2.2 & -4.4 & -9.4 & -6.7 & -1.8 & $1.61 /$ \\
\hline \multirow[t]{2}{*}{ Real } & 0.1 & -1.8 & -7.9 & -1.4 & 4.3 & $7.51 /$ \\
\hline & \multicolumn{6}{|c|}{ (In units indicated) } \\
\hline Dong per U.S. dollar, end of period 2/ & 15,070 & 15,368 & 15,608 & 15,739 & 15,875 & 15957 \\
\hline Dong per U.S. dollar, period average $2 /$ & 14,786 & 15,244 & 15,475 & 15,704 & 15,816 & 15,920 \\
\hline Dong per SDR, end of period & 18,957 & 20,941 & 23,249 & 24,502 & 22,748 & 23,632 \\
\hline \multirow[t]{2}{*}{ Dong per SDR, period average } & 18,746 & 19,785 & 21,695 & 23,281 & 23,429 & 23,246 \\
\hline & \multicolumn{6}{|c|}{ (Annual percentage change, - depreciation) } \\
\hline Dong per U.S. dollar, end of period & -3.8 & -2.0 & -1.6 & -0.8 & -0.9 & $-0.53 /$ \\
\hline Dong per U.S. dollar, period average & -4.3 & -3.1 & -1.5 & -1.5 & -0.7 & $-0.61 /$ \\
\hline Dong per SDR, end of period & -0.2 & -10.5 & -11.0 & -5.4 & 7.2 & $-3.93 /$ \\
\hline Dong per SDR, period average & -0.3 & -5.5 & -9.7 & -7.3 & -0.6 & $2.31 /$ \\
\hline
\end{tabular}

Sources: IMF Information Notice System; and Fund staff estimates.

1/ Compared with Jan-June 2005.

2/ Interbank market rate.

3/ Compared to end-2005. 
Table 23. Vietnam: Balance of Payments, 2002-05

(In millions of U.S. dollars, unless otherwise indicated)

\begin{tabular}{|c|c|c|c|c|}
\hline & 2002 & 2003 & 2004 & 2005 \\
\hline & & & & Prel. \\
\hline Current account balance & -673 & $-1,932$ & $-1,565$ & 218 \\
\hline Excluding official transfers & -827 & $-2,071$ & $-1,740$ & -12 \\
\hline Trade balance & $-1,054$ & $-2,582$ & $-2,287$ & -838 \\
\hline Exports, f.o.b. & 16,706 & 20,149 & 26,485 & 32,442 \\
\hline of which: oil & 3,270 & 3,821 & 5,676 & 7,389 \\
\hline Imports, f.o.b. & 17,760 & 22,730 & 28,772 & 33,280 \\
\hline of which: oil & 2,017 & 2,433 & 3,574 & 4,968 \\
\hline Non-factor services (net) & -749 & -778 & -871 & $-1,106$ \\
\hline Receipts & 2,948 & 3,272 & 3,867 & 4,176 \\
\hline Payments & 3,697 & 4,050 & 4,739 & 5,282 \\
\hline Investment income (net) & -791 & -812 & -891 & $-1,219$ \\
\hline Receipts & 167 & 125 & 188 & 364 \\
\hline Payments & 958 & 937 & 1,079 & 1,583 \\
\hline Transfers (net) & 1,921 & 2,239 & 2,485 & 3,380 \\
\hline Private & 1,767 & 2,100 & 2,310 & 3,150 \\
\hline Official & 154 & 139 & 175 & 230 \\
\hline Capital account balance & 1,136 & 4,083 & 2,447 & 1,913 \\
\hline Gross foreign direct investment (FDI) inflows & 2,023 & 1,894 & 1,878 & 1,972 \\
\hline Equity & 1,882 & 1,294 & 1,283 & 1,347 \\
\hline Loan disbursements & 141 & 600 & 595 & 625 \\
\hline FDI loan repayments & 414 & 590 & 536 & 524 \\
\hline Medium and long-term loans (net) & 523 & 1,045 & 1,396 & 1,405 \\
\hline Disbursements & 1,102 & 1,540 & 1,920 & 1,914 \\
\hline ODA loans & 1,073 & 1,258 & 1,394 & 1,432 \\
\hline Commercial loans & 29 & 283 & 526 & 483 \\
\hline Amortization $1 /$ & 578 & 495 & 523 & 509 \\
\hline Portfolio Investment $2 /$ & $\ldots$ & $\ldots$ & $\ldots$ & 850 \\
\hline Short-term capital (net) $3 /$ & -996 & 1,734 & -291 & $-1,790$ \\
\hline Overall balance & 463 & 2,151 & 883 & 2,131 \\
\hline Financing & -464 & -2151 & -883 & $-2,131$ \\
\hline Change in SBV's NFA (-, increase) & -464 & $-2,151$ & -883 & $-2,131$ \\
\hline \multicolumn{5}{|l|}{ Memorandum items: } \\
\hline Gross official reserves & 3,692 & 5,620 & 6,314 & 8,557 \\
\hline In weeks of next year's imports & 7.2 & 8.7 & 8.5 & 9.8 \\
\hline Ratio to short term external debt (in percent) on remaining maturity basis $2 /$ & 373 & 530 & 611 & 702 \\
\hline Net official international reserves & 2,956 & 4,683 & 5,554 & 7,728 \\
\hline In weeks of next year's imports & 5.8 & 7.3 & 7.5 & 8.9 \\
\hline SBV's net foreign assets & 3,682 & 5,833 & 6,716 & 8,847 \\
\hline Net foreign assets of the banking system & 7,640 & 8,419 & 9,271 & 12,036 \\
\hline In weeks of next year's imports & 14.9 & 13.1 & 12.5 & 13.8 \\
\hline Current account balance (in percent of GDP) & -1.9 & -4.9 & -3.4 & 0.4 \\
\hline Trade deficit (in percent of GDP) & -3.0 & -6.5 & -5.0 & -1.6 \\
\hline Non-oil current account deficit (in percent of GDP) & -5.5 & -8.4 & -8.1 & -4.2 \\
\hline Export value (ann. perc. change) & 11.2 & 20.6 & 31.4 & 22.5 \\
\hline Import value (ann. perc. change) & 22.1 & 28.0 & 26.6 & 15.7 \\
\hline Non-oil export value (ann. perc. change) & 12.9 & 21.5 & 27.4 & 20.4 \\
\hline Non-oil import value (ann. perc. change) & 23.8 & 28.9 & 24.1 & 12.4 \\
\hline
\end{tabular}

Sources: Data provided by the Vietnamese authorities; and Fund staff estimates.

$1 /$ Including two debt buyback operations carried out in 2002 and 2003, respectively.

2/ Includes US\$750 million sovereign bond issue in 2005.

3 / Including net errors and omissions and trade credit. 
Table 24. Vietnam: Merchandise Exports by Commodity, 2001-05

\begin{tabular}{|c|c|c|c|c|c|}
\hline & 2001 & 2002 & 2003 & 2004 & $\frac{2005}{\text { Prel. }}$ \\
\hline & \multicolumn{5}{|c|}{ (In millions of U.S. dollars, unless otherwise indicated) } \\
\hline Total exports, f.o.b. & 15,029 & 16,706 & 20,149 & 26,485 & 32,442 \\
\hline (annual percentage change) & 3.8 & 11.2 & 20.6 & 31.4 & 22.5 \\
\hline (Excluding oil) & 11,901 & 13,436 & 16,328 & 20,814 & 25,069 \\
\hline (annual percentage change) & 8.4 & 12.9 & 21.5 & 27.5 & 20.4 \\
\hline Crude oil & 3,126 & 3,270 & 3,821 & 5,671 & 7373 \\
\hline Volume ('000 tons) & 16,732 & 16,876 & 17,143 & 19,501 & 17967 \\
\hline Unit value (US\$/ton) & 187 & 194 & 223 & 291 & 410 \\
\hline Coal & 113 & 154 & 188 & 354 & 669 \\
\hline Volume ('000 tons) & 4,292 & 6,047 & 7,262 & 11,636 & 17,986 \\
\hline Unit value (US\$/ton) & 26 & 26 & 26 & 30 & 37 \\
\hline Rubber & 166 & 271 & 378 & 596 & 804 \\
\hline Volume ('000 tons) & 308 & 455 & 432 & 513 & 587 \\
\hline Unit value (US\$/ton) & 539 & 595 & 874 & 1,162 & 1,370 \\
\hline Rice & 624 & 726 & 720 & 950 & 1,407 \\
\hline Volume ('000 tons) & 3,721 & 3,236 & 3,810 & 4,063 & 5,250 \\
\hline Unit value (US\$/ton) & 168 & 224 & 189 & 234 & 268 \\
\hline Coffee & 391 & 322 & 505 & 642 & 735 \\
\hline Volume ('000 tons) & 931 & 722 & 749 & 976 & 892 \\
\hline Unit value (US\$/ton) & 420 & 445 & 674 & 658 & 825 \\
\hline Marine products (including frozen items) & 1,816 & 2,022 & 2,200 & 2,408 & 2,739 \\
\hline Garments & 1,975 & 2,732 & 3,609 & 4,430 & 4,838 \\
\hline Footwear & 1,587 & 1,875 & 2,261 & 2,691 & 3,040 \\
\hline Handicrafts & 309 & 340 & 397 & 508 & 436 \\
\hline Electronic goods and components & 709 & 605 & 855 & 1,062 & 1,427 \\
\hline \multirow[t]{2}{*}{ Other } & 4,212 & 4,389 & 5,216 & 7,172 & 8,973 \\
\hline & \multicolumn{5}{|c|}{ (In percent of value of total exports) } \\
\hline Crude oil & 20.8 & 19.6 & 19.0 & 21.4 & 22.7 \\
\hline Coal & 0.8 & 0.9 & 0.9 & 1.3 & 2.1 \\
\hline Rubber & 1.1 & 1.6 & 1.9 & 2.3 & 2.5 \\
\hline Rice & 4.1 & 4.3 & 3.6 & 3.6 & 4.3 \\
\hline Coffee & 2.6 & 1.9 & 2.5 & 2.4 & 2.3 \\
\hline Marine products & 12.1 & 12.1 & 10.9 & 9.1 & 8.4 \\
\hline Garments & 13.1 & 16.4 & 17.9 & 16.7 & 14.9 \\
\hline Footwear & 10.6 & 11.2 & 11.2 & 10.2 & 9.4 \\
\hline Handicrafts & 2.1 & 2.0 & 2.0 & 1.9 & 1.3 \\
\hline Electronic goods and components & 4.7 & 3.6 & 4.2 & 4.0 & 4.4 \\
\hline Other & 28.0 & 26.3 & 25.9 & 27.1 & 27.7 \\
\hline
\end{tabular}

Source: General Statistics Office. 
Table 25. Vietnam: Merchandise Imports by Commodity, 2001-05

\begin{tabular}{|c|c|c|c|c|c|}
\hline & 2001 & 2002 & 2003 & 2004 & $\frac{2005}{\text { Prel. }}$ \\
\hline & \multicolumn{5}{|c|}{ (In millions of U.S. dollars, unless otherwise indicated) } \\
\hline Total imports, c.i.f. & 16,218 & 19,746 & 25,256 & 31,969 & 36,978 \\
\hline (annual percentage change) & 3.7 & 21.8 & 27.9 & 26.6 & 15.7 \\
\hline Petroleum products (gasoline, diesel, etc.) & 1,834 & 2,023 & 2,441 & 3,572 & 5,024 \\
\hline Volume ('000 tons) & 9,083 & 9,970 & 9,936 & 11,048 & 11477 \\
\hline Unit value (US\$/ton) & 202 & 203 & 246 & 323 & 438 \\
\hline Fertilizer & 418 & 478 & 631 & 759 & 641 \\
\hline Volume ('000 tons) & 3,288 & 3,820 & 4,135 & 4,065 & 2877 \\
\hline Unit value (US\$/ton) & 127 & 125 & 153 & 187 & 223 \\
\hline Insecticide & 103 & 116 & 116.1 & 143 & 244 \\
\hline Volume ('000 tons) & $\ldots$ & $\ldots$ & $\ldots$ & $\ldots$ & \\
\hline Unit value (US\$/ton) & $\ldots$ & $\ldots$ & $\ldots$ & $\ldots$ & \\
\hline Steel and iron & 965 & 1,334 & 1,695 & 2,553 & 2931 \\
\hline Volume ('000 tons) & 3,870 & 4,946 & 4,623 & 5,152 & 5524 \\
\hline Unit value (US\$/ton) & 249 & 270 & 367 & 496 & 531 \\
\hline Motorcycles & 635 & 423 & 329 & 452 & 541 \\
\hline Volume ('000 unit) & 2,380 & 1,480 & $\ldots$ & $\ldots$ & $\ldots$ \\
\hline Unit value (US\$/unit) & 267 & 286 & $\ldots$ & $\ldots$ & $\ldots$ \\
\hline Motor cars and trucks & 199 & 262 & 275 & 360 & 285 \\
\hline Volume ('000 units) & 28.3 & 29.4 & 21.4 & 25.0 & 17.3 \\
\hline Unit value (US\$/unit) & 7,051 & 8,911 & 12,863 & 14,420 & 16,402 \\
\hline Wheat flour & 12 & 11 & 10 & 10 & 9 \\
\hline Volume ('000 tons) & 66 & 62 & 52 & 48 & 39 \\
\hline Unit value (US\$/ton) & 181 & 184 & 187 & 209 & 220 \\
\hline Textile yarn (fibers, spun) & 228 & 273 & 317 & 384 & 340 \\
\hline Volume ('000 tons) & $\ldots$ & $\ldots$ & $\ldots$ & $\ldots$ & $\ldots$ \\
\hline Unit value (US\$/ton) & $\cdots$ & $\ldots$ & $\cdots$ & $\ldots$ & $\cdots$ \\
\hline Cotton & 115 & 112 & 105 & 192 & 167 \\
\hline Leather and garment material & 1,590 & 1,711 & 2,034 & 1,350 & 2282 \\
\hline Cigarette material (including tobacco) & $\ldots$ & $\ldots$ & $\ldots$ & $\ldots$ & \\
\hline Machinery and equipment (including aircraft) & 2,741 & 3,793 & 5,359 & 5,249 & 5281 \\
\hline \multirow[t]{2}{*}{ Other } & 7,377 & 9,210 & 11,944 & 16,945 & 19,235 \\
\hline & \multicolumn{5}{|c|}{ (In percent of value of total imports) } \\
\hline Petroleum products & 11.3 & 10.2 & 9.7 & 11.2 & 13.6 \\
\hline Fertilizer & 2.6 & 2.4 & 2.5 & 2.4 & 1.7 \\
\hline Insecticide & 0.6 & 0.6 & 0.5 & 0.4 & 0.7 \\
\hline Steel and iron & 6.0 & 6.8 & 6.7 & 8.0 & 7.9 \\
\hline Motorcycles & 3.9 & 2.1 & 1.3 & 1.4 & 1.5 \\
\hline Motor cars and trucks & 1.2 & 1.3 & 1.1 & 1.1 & 0.8 \\
\hline Wheat flour & $\ldots$ & $\ldots$ & $\ldots$ & $\ldots$ & $\ldots$ \\
\hline Textile yarn & 1.4 & 1.4 & 1.3 & 1.2 & 0.9 \\
\hline Cotton & 0.7 & 0.6 & 0.4 & 0.6 & 0.5 \\
\hline Leather and garment material & 9.8 & 8.7 & 8.1 & 4.2 & 6.2 \\
\hline Cigarette material & $\ldots$ & $\ldots$ & $\ldots$ & $\ldots$ & $\ldots$ \\
\hline Machinery and equipment & 16.9 & 19.2 & 21.2 & 16.4 & 14.3 \\
\hline Other & 45.5 & 46.6 & 47.3 & 53.0 & 52.0 \\
\hline
\end{tabular}

Source: General Statistics Office. 
Table 26. Vietnam: Direction of Trade, 2001-05

(In percent of total exports or imports)

\begin{tabular}{|c|c|c|c|c|c|}
\hline & 2001 & 2002 & 2003 & 2004 & $\frac{2005}{\text { Prel. }}$ \\
\hline \multicolumn{6}{|l|}{ Exports } \\
\hline Industrial countries or regions & 64.1 & 68.2 & 71.0 & 68.6 & 66.5 \\
\hline Japan & 16.7 & 14.6 & 14.4 & 13.4 & 13.6 \\
\hline Hong Kong SAR & 2.1 & 2.0 & 1.8 & 1.4 & 1.1 \\
\hline Korea & 2.7 & 2.8 & 2.4 & 2.3 & 1.9 \\
\hline Singapore & 7.0 & 5.8 & 5.1 & 5.6 & 5.6 \\
\hline France & 3.1 & 2.6 & 2.5 & 2.1 & 2.0 \\
\hline Germany & 4.8 & 4.4 & 4.2 & 4.0 & 3.3 \\
\hline Italy & 1.6 & 1.6 & 1.6 & 1.4 & 1.4 \\
\hline Netherlands & 2.4 & 2.4 & 2.4 & 2.2 & 2.0 \\
\hline Switzerland & 0.6 & 0.4 & 0.4 & 0.5 & 0.3 \\
\hline United Kingdom & 3.4 & 3.4 & 3.7 & 3.8 & 3.1 \\
\hline Other Europe (industrialized) 1/ & 4.8 & 4.6 & 4.7 & 4.7 & 4.5 \\
\hline United States & 7.1 & 14.7 & 19.5 & 19.0 & 18.3 \\
\hline Canada & 0.7 & 0.8 & 0.9 & 1.0 & 1.1 \\
\hline Australia & 6.9 & 8.0 & 7.1 & 7.1 & 7.9 \\
\hline New Zealand & 0.1 & 0.1 & 0.1 & 0.2 & 0.1 \\
\hline Developing countries & 35.9 & 31.8 & 29.0 & 31.4 & 33.5 \\
\hline China (mainland) & 9.4 & 9.1 & 9.3 & 10.9 & 9.1 \\
\hline Indonesia & 1.8 & 2.0 & 2.3 & 1.7 & 1.4 \\
\hline Malaysia & 2.7 & 2.1 & 2.3 & 2.4 & 2.9 \\
\hline Philippines & 2.5 & 1.9 & 1.7 & 1.9 & 2.6 \\
\hline Russia & 1.3 & 1.1 & 0.8 & 0.8 & 0.8 \\
\hline Taiwan POC & 5.4 & 4.9 & 3.7 & 3.4 & 2.9 \\
\hline Thailand & 2.1 & 1.4 & 1.7 & 2.0 & 2.4 \\
\hline Other & 11.2 & 9.4 & 7.3 & 8.3 & 11.4 \\
\hline \multicolumn{6}{|l|}{ Imports } \\
\hline Industrial countries or regions & 59.0 & 55.6 & 54.8 & 52.3 & 50.3 \\
\hline Japan & 13.5 & 12.7 & 11.8 & 11.1 & 11.1 \\
\hline Hong Kong SAR & 3.3 & 4.1 & 3.9 & 3.4 & 3.3 \\
\hline Korea & 11.6 & 11.5 & 10.4 & 10.5 & 9.7 \\
\hline Singapore & 15.3 & 12.8 & 11.4 & 11.3 & 12.4 \\
\hline France & 1.9 & 1.5 & 1.6 & 1.9 & 1.2 \\
\hline Germany & 2.4 & 2.8 & 2.4 & 2.2 & 1.8 \\
\hline Italy & 1.2 & 1.4 & 1.5 & 1.0 & 0.8 \\
\hline Netherlands & 0.7 & 0.6 & 1.3 & 0.6 & 0.8 \\
\hline Switzerland & 0.6 & 0.7 & 1.2 & 2.1 & 2.4 \\
\hline United Kingdom & 1.1 & 0.8 & 0.9 & 0.7 & 0.5 \\
\hline Other Europe (industrialized) 1/ & 2.1 & 2.2 & 2.2 & 1.8 & 1.7 \\
\hline United States & 2.5 & 2.3 & 4.5 & 3.5 & 2.3 \\
\hline Canada & 0.4 & 0.3 & 0.3 & 0.3 & 0.5 \\
\hline Australia & 1.6 & 1.4 & 1.1 & 1.4 & 1.3 \\
\hline New Zealand & 0.9 & 0.3 & 0.4 & 0.3 & 0.3 \\
\hline Developing countries & 41.0 & 44.4 & 45.2 & 47.7 & 49.7 \\
\hline China (mainland) & 9.9 & 10.9 & 12.4 & 14.4 & 15.6 \\
\hline Indonesia & 1.8 & 1.8 & 2.2 & 2.1 & 1.9 \\
\hline Malaysia & 2.9 & 3.5 & 3.7 & 3.8 & 3.4 \\
\hline Philippines & 0.3 & 0.5 & 0.6 & 0.6 & 0.6 \\
\hline Russia & 2.3 & 2.5 & 1.9 & 2.1 & 2.1 \\
\hline Taiwan POC & 12.4 & 12.8 & 11.5 & 11.6 & 11.7 \\
\hline Thailand & 4.9 & 4.8 & 5.1 & 5.8 & 6.5 \\
\hline Other & 6.5 & 7.5 & 7.8 & 7.4 & 8.0 \\
\hline \multicolumn{6}{|l|}{ Memorandum items: } \\
\hline \multicolumn{6}{|l|}{ European Union } \\
\hline Exports & 20.0 & 19.8 & 19.9 & 18.8 & 17.0 \\
\hline Imports & 9.3 & 9.5 & 10.2 & 8.4 & 7.0 \\
\hline \multicolumn{6}{|l|}{ ASEAN } \\
\hline Exports & 17.0 & 14.6 & 14.7 & 15.3 & 16.8 \\
\hline Imports & 25.7 & 24.2 & 23.6 & 24.3 & 25.6 \\
\hline
\end{tabular}

Sources: General Statistics Office; and Fund staff estimates.

1/ Defined as Austria, Belgium, Denmark, Finland, Greece, Iceland, Ireland, Luxembourg, Norway, Portugal, Spain, and Sweden. 
Table 27. Vietnam: Commitments of Foreign Direct Investment, 2001-005 1/

\begin{tabular}{|c|c|c|c|c|c|}
\hline & 2001 & 2002 & 2003 & 2004 & 2005 \\
\hline & \multicolumn{5}{|c|}{ (In millions of U.S. dollars) } \\
\hline Industry & 2,366 & 2,043 & 2,235 & 2,741 & 4,510 \\
\hline Heavy industries & 1,490 & 868 & 854 & 1,584 & 2,895 \\
\hline Export processing zones & 30 & 71 & 18 & 41 & 40 \\
\hline Light industries & 773 & 774 & 1,190 & 943 & 1,285 \\
\hline Food & 74 & 330 & 173 & 173 & 290 \\
\hline Oil and gas & 0 & 45 & 16 & 12 & 20 \\
\hline Construction & 89 & 192 & 101 & 481 & 174 \\
\hline Transportation and communications & 244 & 78 & 19 & 78 & 709 \\
\hline Real estate & 62 & 236 & 315 & 467 & 601 \\
\hline Hotels and tourism & 20 & 218 & 185 & 257 & 124 \\
\hline Office property and apartments (office, new cities) & 42 & 19 & 130 & 210 & 477 \\
\hline Agriculture, forestry, and fisheries (agr, Fisheries) & 253 & 264 & 298 & 537 & 393 \\
\hline Services (service, Culture, finance) & 250 & 134 & 189 & 219 & 427 \\
\hline \multirow[t]{2}{*}{ Total } & 3,265 & 2,993 & 3,172 & 4,534 & 6,835 \\
\hline & \multicolumn{5}{|c|}{ (In percent of total) } \\
\hline Industry & 72.5 & 68.3 & 70.5 & 60.5 & 66.0 \\
\hline Heavy industries & 45.6 & 29.0 & 26.9 & 34.9 & 42.4 \\
\hline Export processing zones & 0.9 & 2.4 & 0.6 & 0.9 & 0.6 \\
\hline Light industries & 23.7 & 25.9 & 37.5 & 20.8 & 18.8 \\
\hline Food & 2.3 & 11.0 & 5.4 & 3.8 & 4.2 \\
\hline Oil and gas & 0.0 & 1.5 & 0.5 & 0.3 & 0.3 \\
\hline Construction & 2.7 & 6.4 & 3.2 & 10.6 & 2.5 \\
\hline Transportation and communications & 7.5 & 2.6 & 0.6 & 1.7 & 10.4 \\
\hline Real estate & 1.9 & 7.9 & 9.9 & 10.3 & 8.8 \\
\hline Hotels and tourism & 0.6 & 7.3 & 5.8 & 5.7 & 1.8 \\
\hline Office property and apartments & 1.3 & 0.6 & 4.1 & 4.6 & 7.0 \\
\hline Agriculture, forestry, and fisheries & 7.8 & 8.8 & 9.4 & 11.8 & 5.8 \\
\hline Services & 7.7 & 4.5 & 6.0 & 4.8 & 6.2 \\
\hline Total & 100.0 & 100.0 & 100.0 & 100.0 & 100.0 \\
\hline
\end{tabular}

Source: Ministry of Planning and Investment.

$1 /$ Includes investments by domestic joint venture partners. Commitments include both new projects and additional capital of ongoing projects. 
Table 28. Vietnam: Public Sector Debt, 2002-05 1/

\begin{tabular}{|c|c|c|c|c|}
\hline & 2002 & 2003 & 2004 & $\begin{array}{r}2005 \\
\text { Prel. }\end{array}$ \\
\hline & \multicolumn{4}{|c|}{ (In billions of dong) } \\
\hline Public and publicly guaranteed debt & 204,723 & 252,101 & 305,663 & 368,992 \\
\hline Domestic debt & 54,363 & 84,134 & 109,972 & 145,844 \\
\hline T-bills and bonds & 34,728 & 42,795 & 50,649 & 63,019 \\
\hline maturity less than 1 year & 6,089 & 12,118 & 14,220 & 17,886 \\
\hline maturity greater than 1 year & 28,639 & 30,677 & 36,429 & 45,133 \\
\hline Disbursements & 15,210 & 22,895 & 27,452 & 33,250 \\
\hline maturity less than 1 year & 6,089 & 12,118 & 14,222 & 17,886 \\
\hline maturity greater than 1 year & 9,121 & 10,777 & 13,230 & 15,364 \\
\hline Amortization & 10,499 & 14,828 & 19,596 & 20,050 \\
\hline maturity less than 1 year & 3,422 & 6,089 & 12,118 & 14,220 \\
\hline maturity greater than 1 year & 7,077 & 8,739 & 7,478 & 5,830 \\
\hline Interest & 2,494 & 2,189 & 4,056 & 5,440 \\
\hline maturity less than 1 year & 253 & 475 & 957 & 1,150 \\
\hline maturity greater than 1 year & 2,241 & 1,714 & 3,099 & 4,290 \\
\hline Development Assistance Fund (DAF) 2/ & 14,735 & 23,890 & 32,800 & 38,801 \\
\hline Education bonds & 0 & 2,579 & 2,579 & 5,395 \\
\hline Reform bonds & 4,900 & 8,050 & 9,540 & 9,540 \\
\hline Infrastructure bonds & 0 & 5,020 & 10,040 & 20,625 \\
\hline Municipal bonds & 0 & 1,800 & 4,364 & 7,864 \\
\hline Domestic guarantees & $\ldots$ & $\ldots$ & $\ldots$ & 600 \\
\hline External Debt in billions of dong & 150,360 & 167,967 & 195,692 & 223,148 \\
\hline Sovereign debt & 141,906 & 156,551 & 175,835 & 204,353 \\
\hline External guarantees & 6,969 & 9,665 & 17,516 & 15,980 \\
\hline \multirow[t]{2}{*}{ Short term publicly guaranteed } & 1,486 & 1,751 & 2,341 & 2,815 \\
\hline & \multicolumn{4}{|c|}{ (In percent of GDP) } \\
\hline Public and publicly guaranteed debt & 38.2 & 41.1 & 42.7 & 44.0 \\
\hline Domestic debt & 10.1 & 13.7 & 15.4 & 17.4 \\
\hline T-bills and bonds & 6.5 & 7.0 & 7.1 & 7.5 \\
\hline Development Assistance Fund (DAF) 2/ & 2.8 & 3.9 & 4.6 & 4.6 \\
\hline Education bonds & 0.0 & 0.4 & 0.4 & 0.6 \\
\hline Reform Bonds & 0.9 & 1.3 & 1.3 & 1.1 \\
\hline Infrastructure bonds & 0.0 & 0.8 & 1.4 & 2.5 \\
\hline Municipal bonds & 0.0 & 0.3 & 0.6 & 0.9 \\
\hline Domestic guarantees & $\cdots$ & $\cdots$ & $\ldots$ & 0.1 \\
\hline External Debt & 28.1 & 27.4 & 27.4 & 26.6 \\
\hline Sovereign debt & 26.5 & 25.5 & 24.6 & 24.4 \\
\hline External guarantees & 1.3 & 1.6 & 2.4 & 1.9 \\
\hline Short term publicly guaranteed & 0.3 & 0.3 & 0.3 & 0.3 \\
\hline
\end{tabular}

Sources: Ministry of Finance; and Fund staff estimates.

1/ Data are not fully comparable to the data in the staff report due to revisions as of September 20, 2006 2/ Includes DAF bonds as well as liabilities to the Postal Savings Service Company and Social Security Fund; capital mobilized by branches (deposits); and Project Bonds. 
Table 29. Vietnam: External Debt and Debt Service, 2002-05 1/ (In millions of U.S. Dollars)

\begin{tabular}{|c|c|c|c|c|}
\hline & 2002 & 2003 & 2004 & $\frac{2005}{\text { Prel. }}$ \\
\hline \multicolumn{5}{|l|}{ A. External Debt } \\
\hline $\begin{array}{l}\text { Total External Debt } \\
\text { in percent of GDP }\end{array}$ & $\begin{array}{r}12,345 \\
35.2\end{array}$ & $\begin{array}{r}13,535 \\
34.2\end{array}$ & $\begin{array}{r}15,390 \\
33.9\end{array}$ & $\begin{array}{r}16,924 \\
32.0\end{array}$ \\
\hline $\begin{array}{l}\text { Public and publicly guaranteed } 2 / \\
\text { in percent of GDP }\end{array}$ & $\begin{array}{r}9,887 \\
28.2\end{array}$ & $\begin{array}{r}11,001 \\
27.8\end{array}$ & $\begin{array}{r}12,397 \\
27.3\end{array}$ & $\begin{array}{r}13,809 \\
26.1\end{array}$ \\
\hline $\begin{array}{l}\text { Private } \\
\text { in percent of GDP }\end{array}$ & $\begin{array}{r}2,458 \\
7.0\end{array}$ & $\begin{array}{r}2,534 \\
6.4\end{array}$ & $\begin{array}{r}2,993 \\
6.6\end{array}$ & $\begin{array}{r}3,114 \\
5.9\end{array}$ \\
\hline Medium and Long Term & 12,183 & 13,346 & 15,142 & 16,628 \\
\hline Public and publicly guaranteed $2 /$ & 9,790 & 10,888 & 12,248 & 13,632 \\
\hline Public & 9,292 & 10,091 & 11,159 & 12,632 \\
\hline Multilateral & 3,418 & 4,151 & 4,618 & 5,204 \\
\hline Bilateral & 5,357 & 5,431 & 6,044 & 6,210 \\
\hline Commercial & 517 & 509 & 498 & 1,218 \\
\hline Publicly guaranteed 3/ & 498 & 797 & 1,089 & 1,000 \\
\hline Private & 2,393 & 2,458 & 2,894 & 2,996 \\
\hline Short-term & 162 & 188 & 248 & 296 \\
\hline Public and publicly guaranteed & 97 & 113 & 149 & 178 \\
\hline Public & $\ldots$ & $\ldots$ & $\ldots$ & 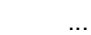 \\
\hline Publicly guaranteed & 97 & 113 & 149 & 178 \\
\hline Private & 65 & 75 & 99 & 118 \\
\hline \multicolumn{5}{|l|}{ B. External Debt Service } \\
\hline Total External Debt Service & 1,630 & 1,764 & 1,858 & 1,952 \\
\hline Amortization 4/ & 1,339 & 1,477 & 1,441 & 1,513 \\
\hline Interest & 291 & 287 & 417 & 439 \\
\hline Total Public and Guaranteed Debt Service 2/ & 776 & 699 & 771 & 722 \\
\hline Amortization & 567 & 489 & 447 & 431 \\
\hline Interest & 208 & 210 & 324 & 291 \\
\hline Total Public Debt Service & 605 & 628 & 522 & 555 \\
\hline Amortization 4/ & 419 & 435 & 227 & 310 \\
\hline Interest & 186 & 194 & 295 & 245 \\
\hline Amortization & 1,339 & 1,477 & 1,441 & 1,513 \\
\hline Medium and Long Term & 992 & 1,085 & 993 & 982 \\
\hline Public and publicly guaranteed $2 /$ & 567 & 489 & 447 & 431 \\
\hline Public & 419 & 435 & 227 & 310 \\
\hline Multilateral & 11 & 16 & $\ldots$ & $\ldots$ \\
\hline Bilateral 4/ & 397 & 409 & $\ldots$ & $\ldots$ \\
\hline Commercial & 10 & 10 & $\ldots$ & $\ldots$ \\
\hline Publicly guaranteed & 149 & 54 & 220 & 121 \\
\hline Private & 425 & 597 & 546 & 550 \\
\hline Interest & 291 & 287 & 417 & 439 \\
\hline Medium and Long Term & 291 & 287 & 417 & 439 \\
\hline Public and publicly guaranteed $2 /$ & 208 & 210 & 324 & 291 \\
\hline Public & 186 & 194 & 295 & 245 \\
\hline Multilateral & 23 & 33 & $\ldots$ & $\ldots$ \\
\hline Bilateral & 142 & 145 & $\ldots$ & $\ldots$ \\
\hline Commercial & 22 & 16 & $\ldots$ & $\ldots$ \\
\hline Publicly guaranteed & 22 & 16 & 29 & 46 \\
\hline Private & 82 & 77 & 93 & 148 \\
\hline \multicolumn{5}{|l|}{ Memorandum item: } \\
\hline GDP in millions of USD & 35,082 & 39,542 & 45,441 & 52,838 \\
\hline
\end{tabular}

Sources: Ministry of Finance; and Fund staff estimates.

1/ Data are not fully comparable to the data in the staff report due to revisions as of September 20, 2006.

2/ Includes loans to State-owned-enterprises not classified elsewhere.

$3 /$ Short-term debt of State-owned-enterprises.

4/ 2002-03 data reflect debt buyback operations. 


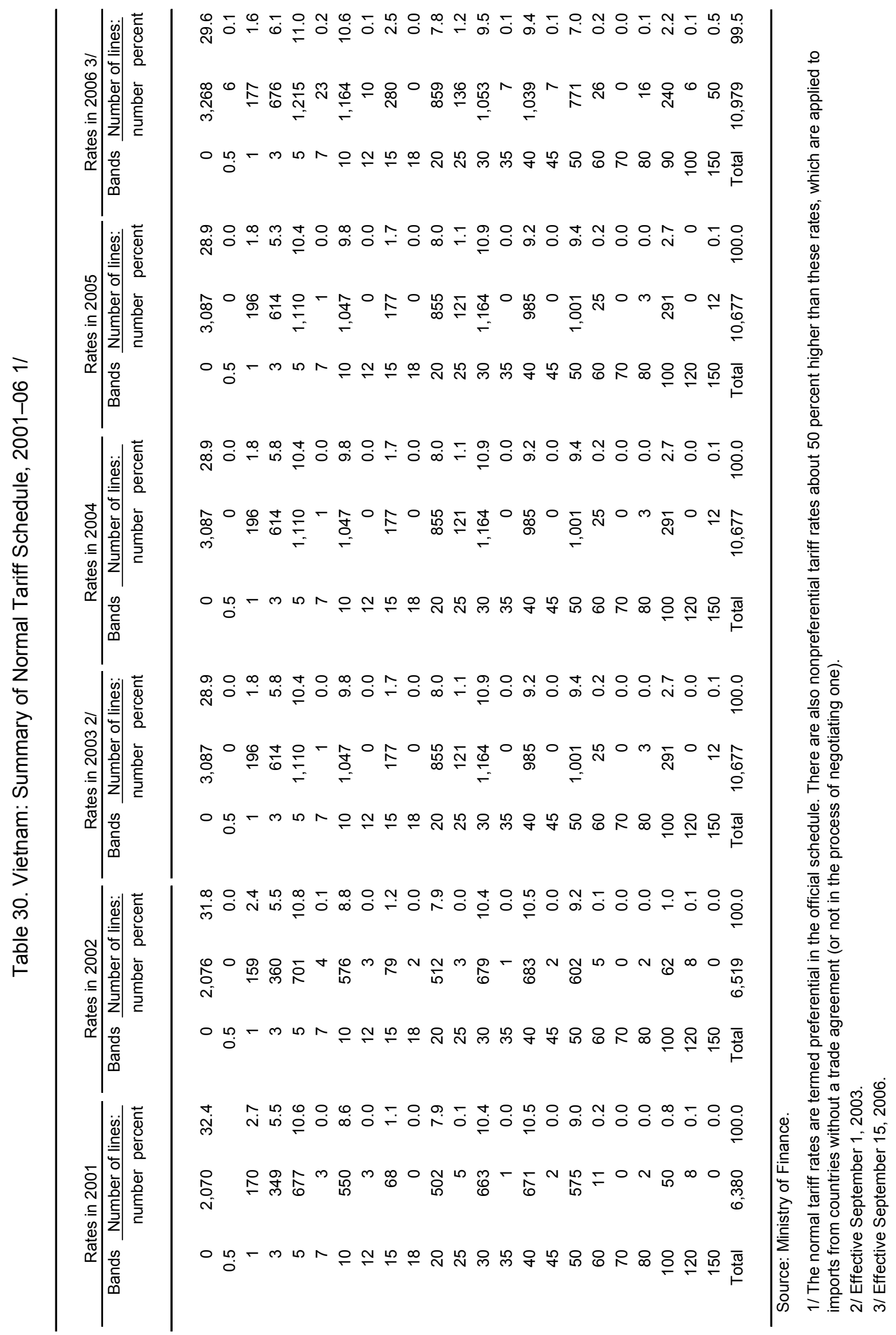




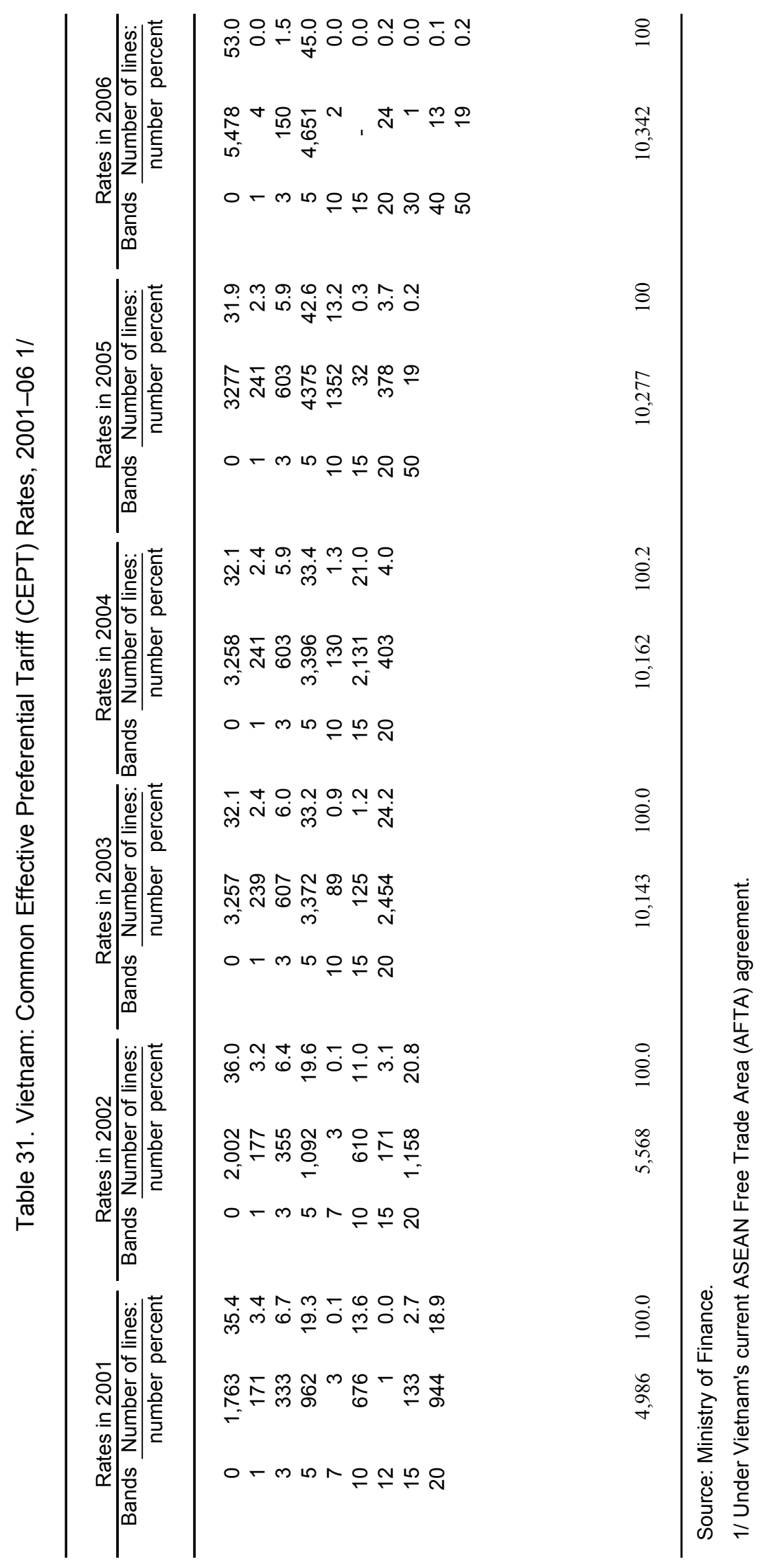




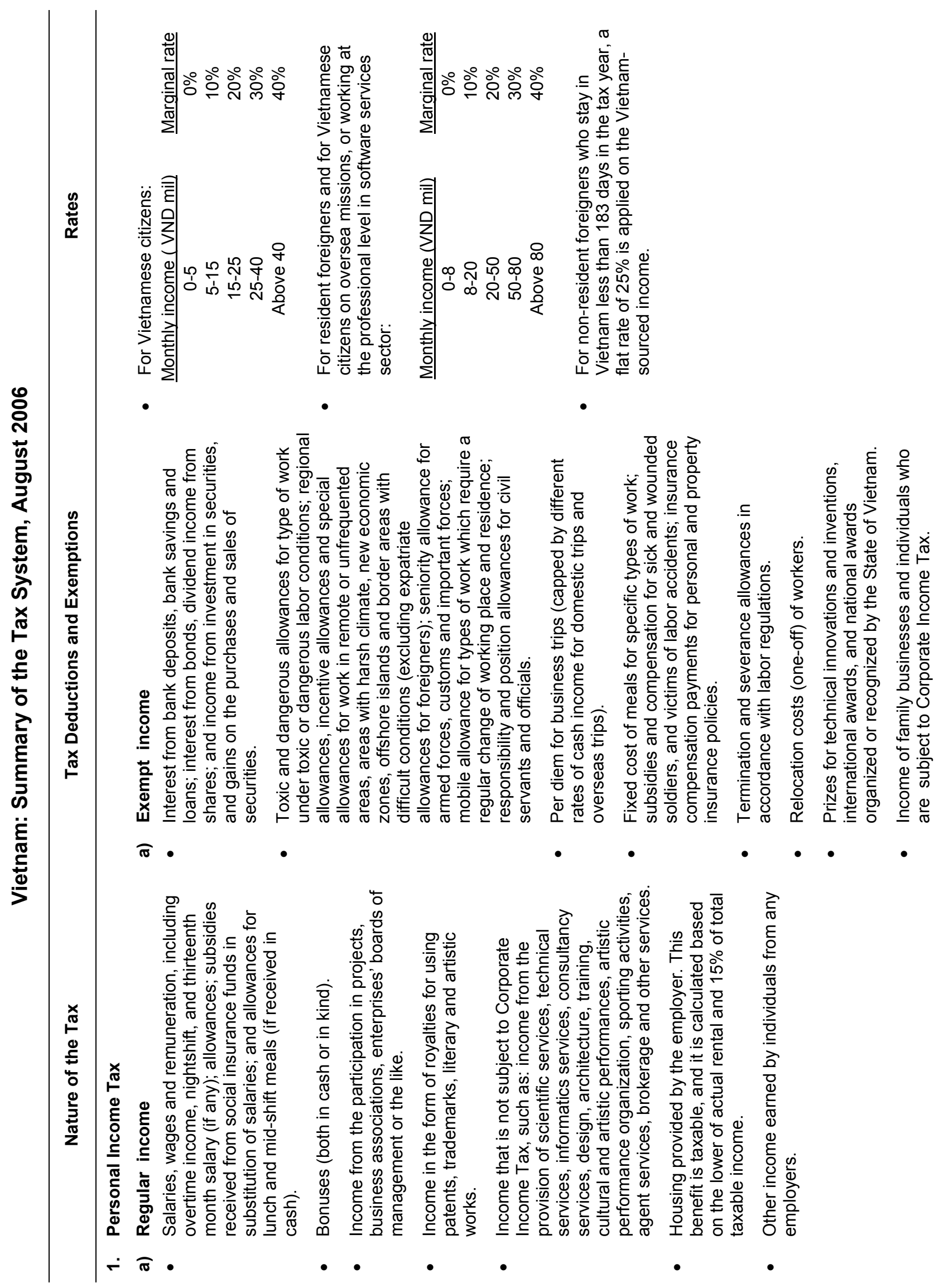




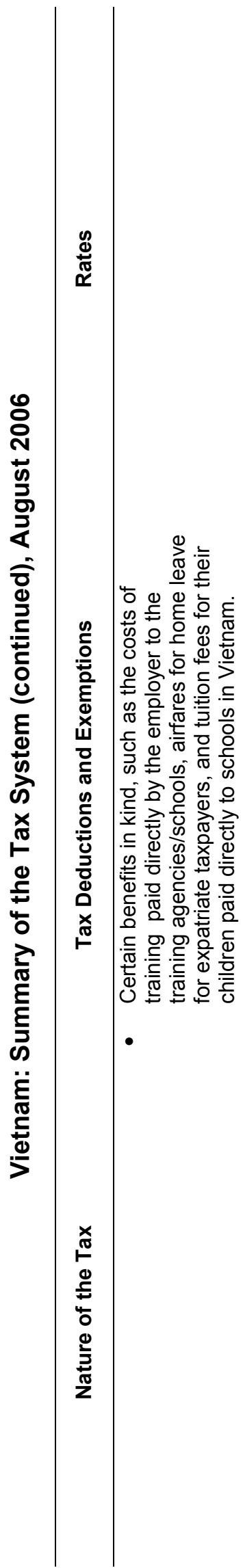

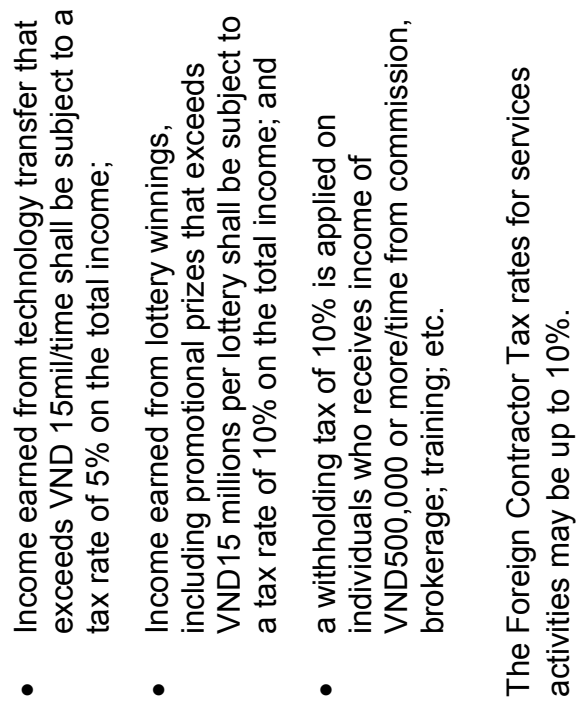
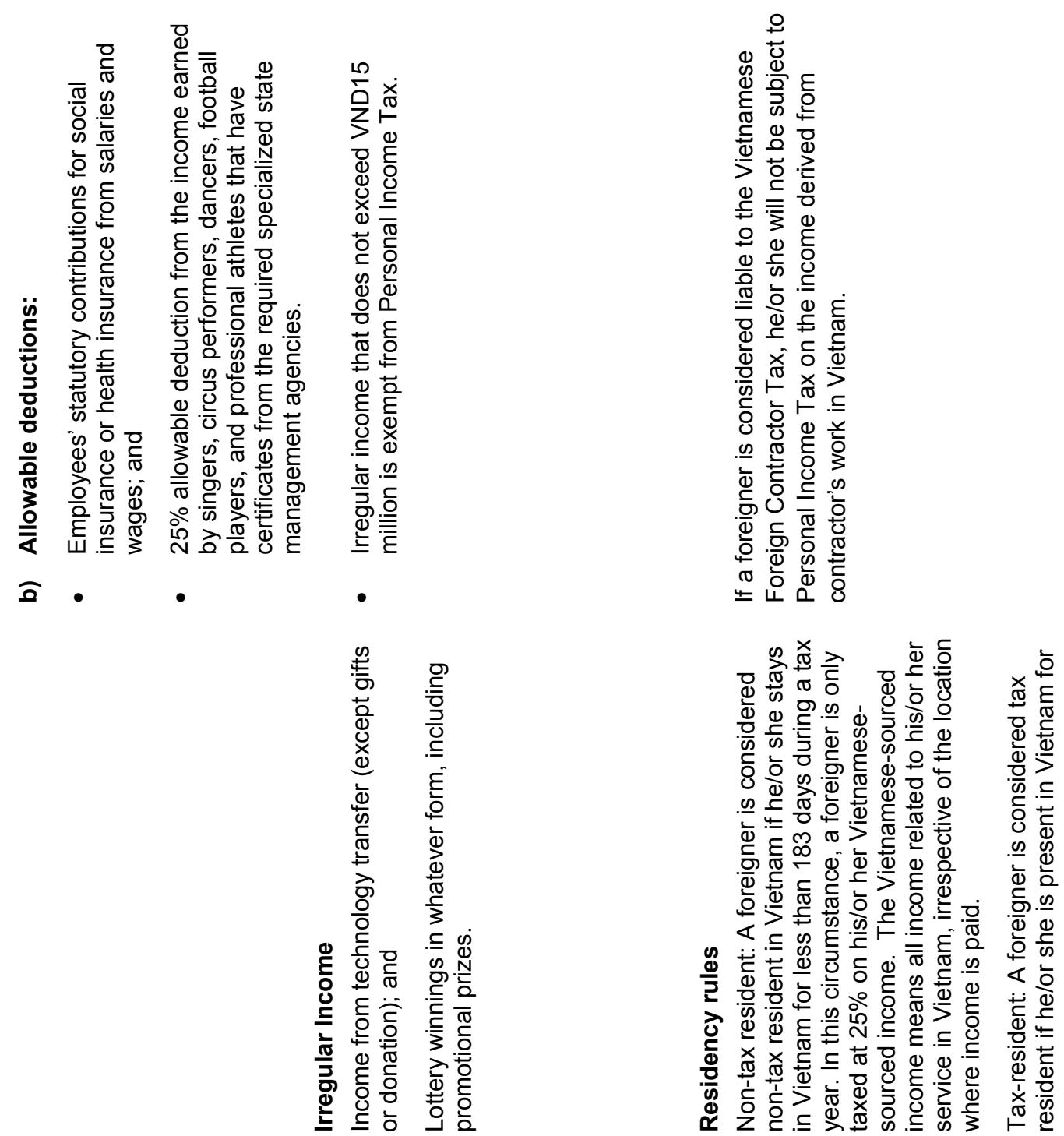


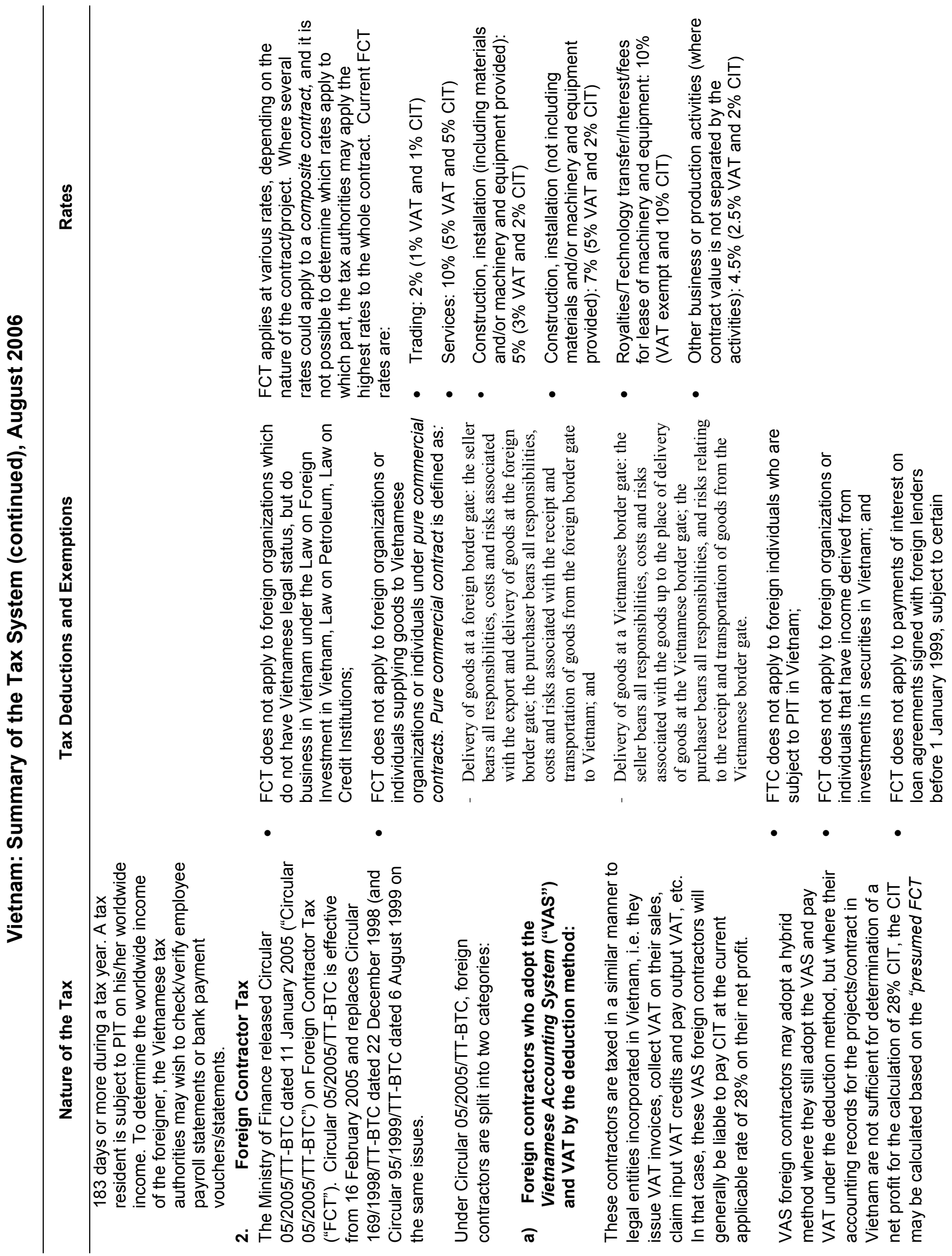




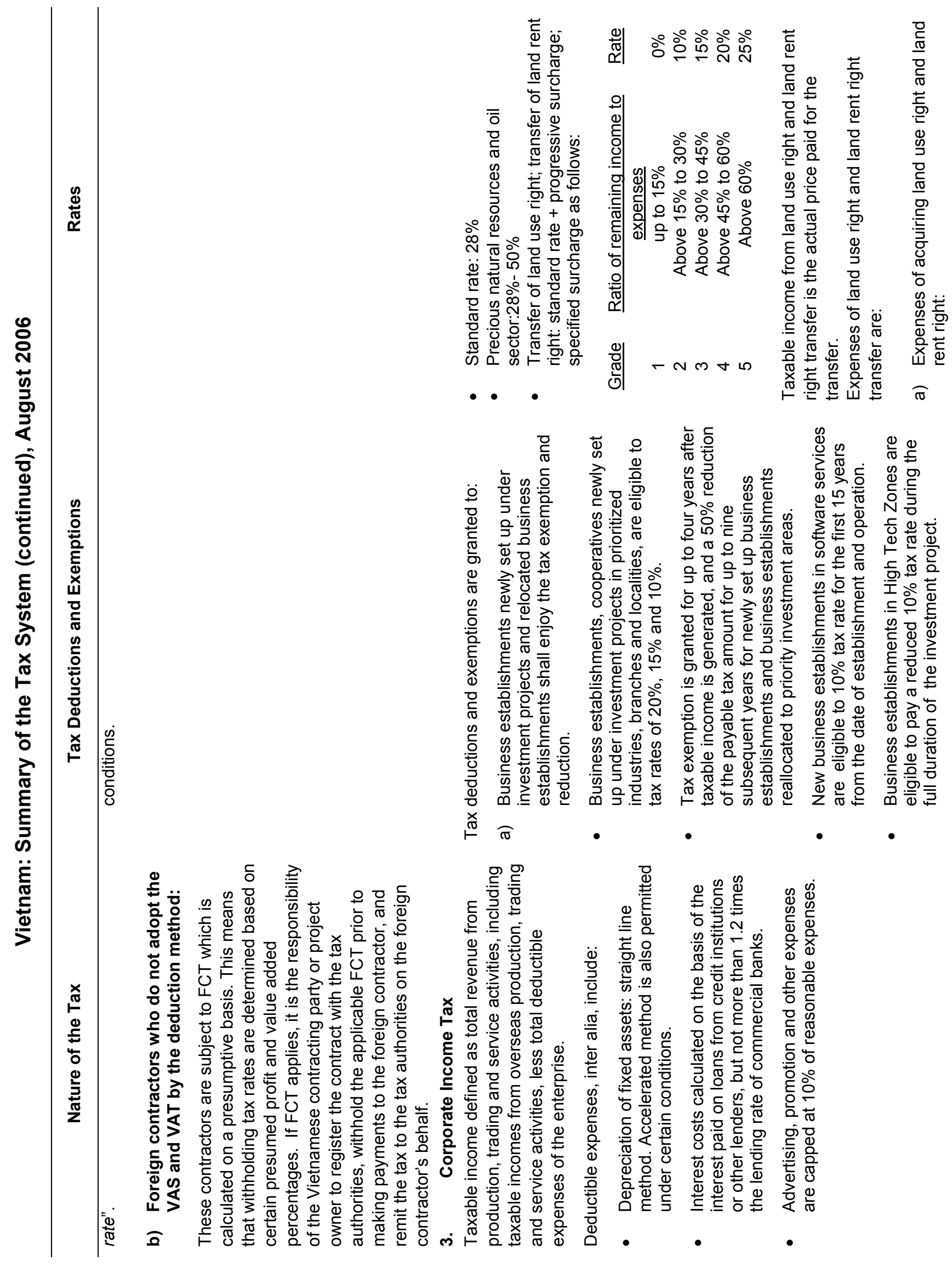




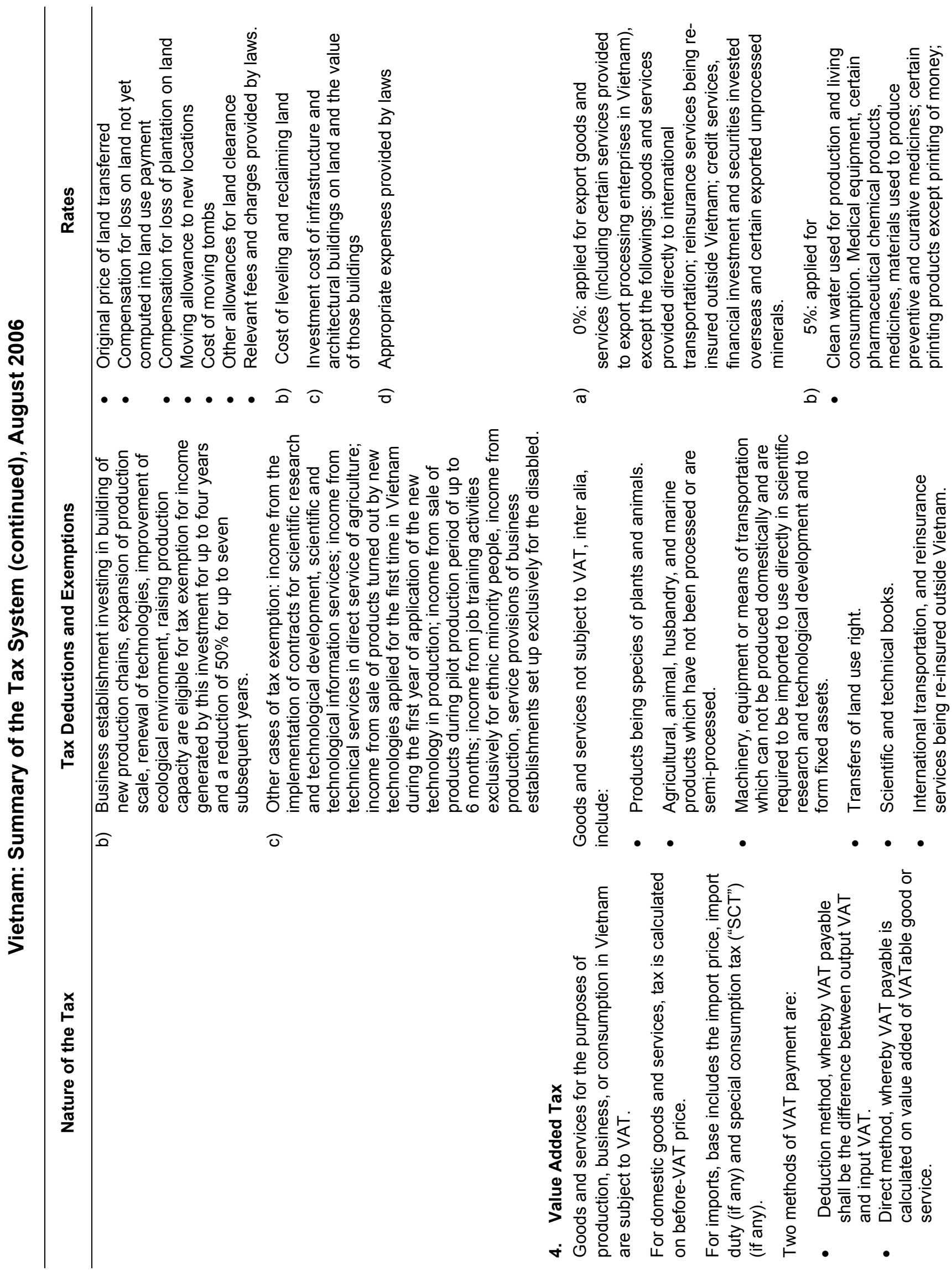




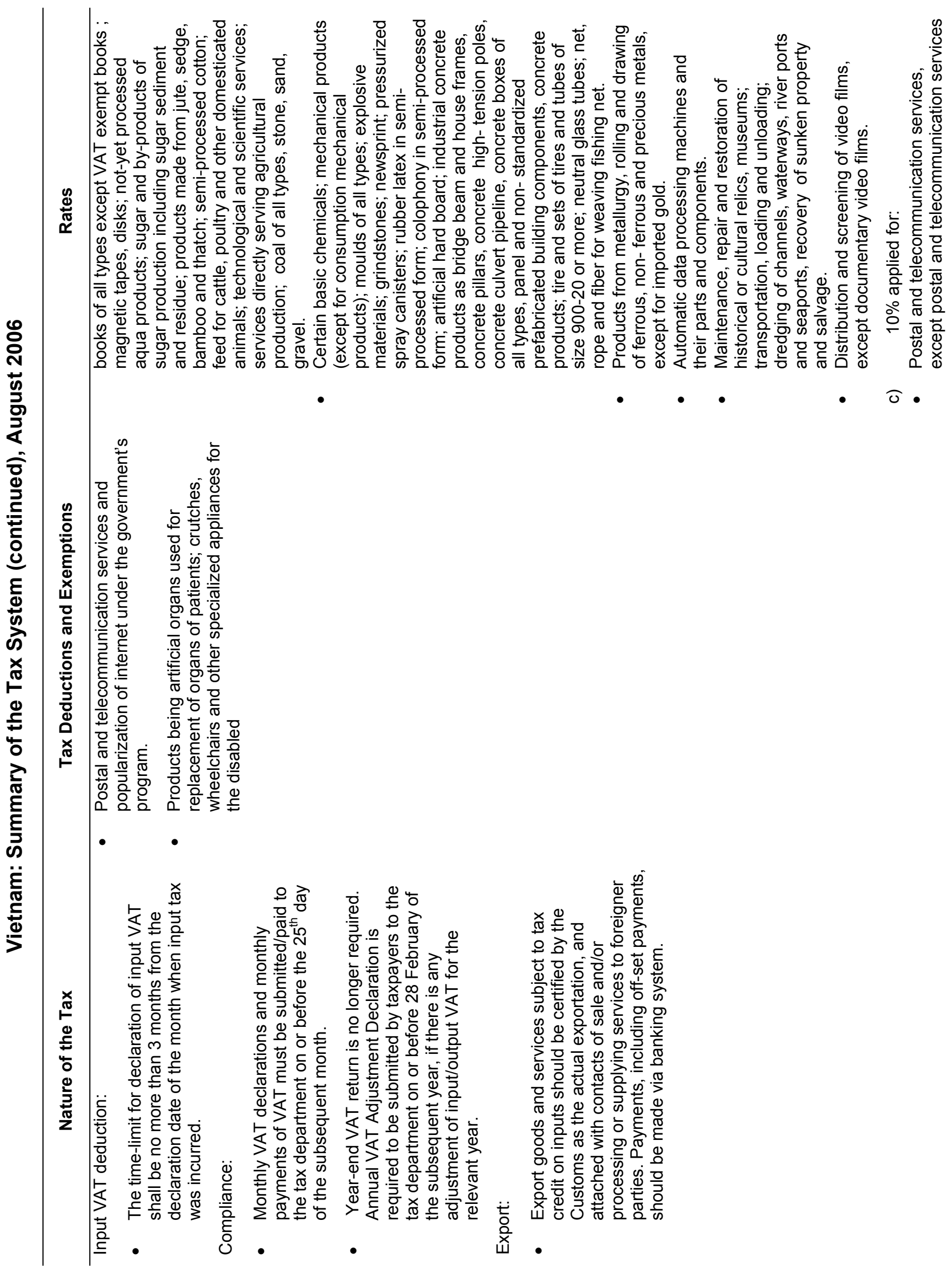




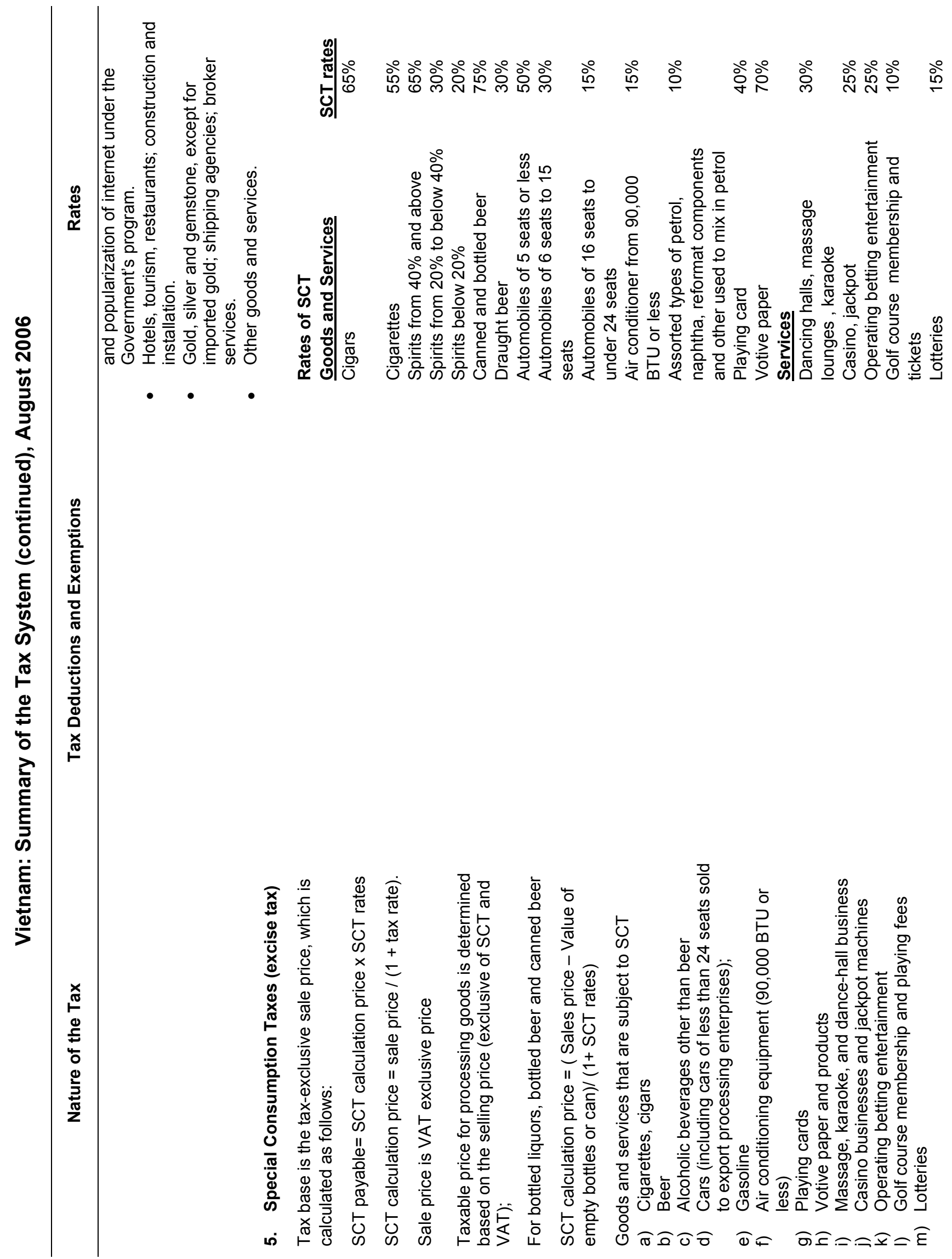




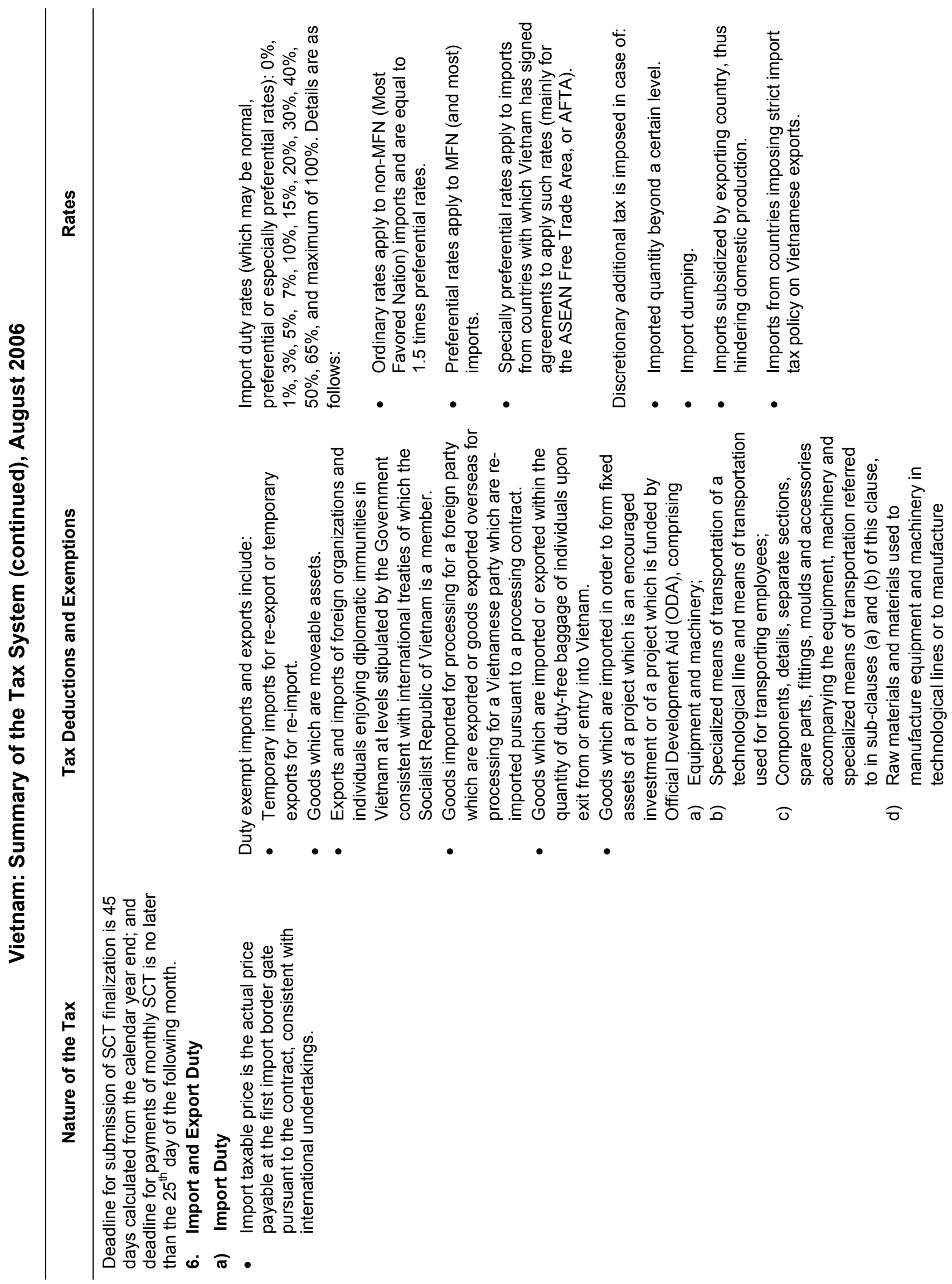



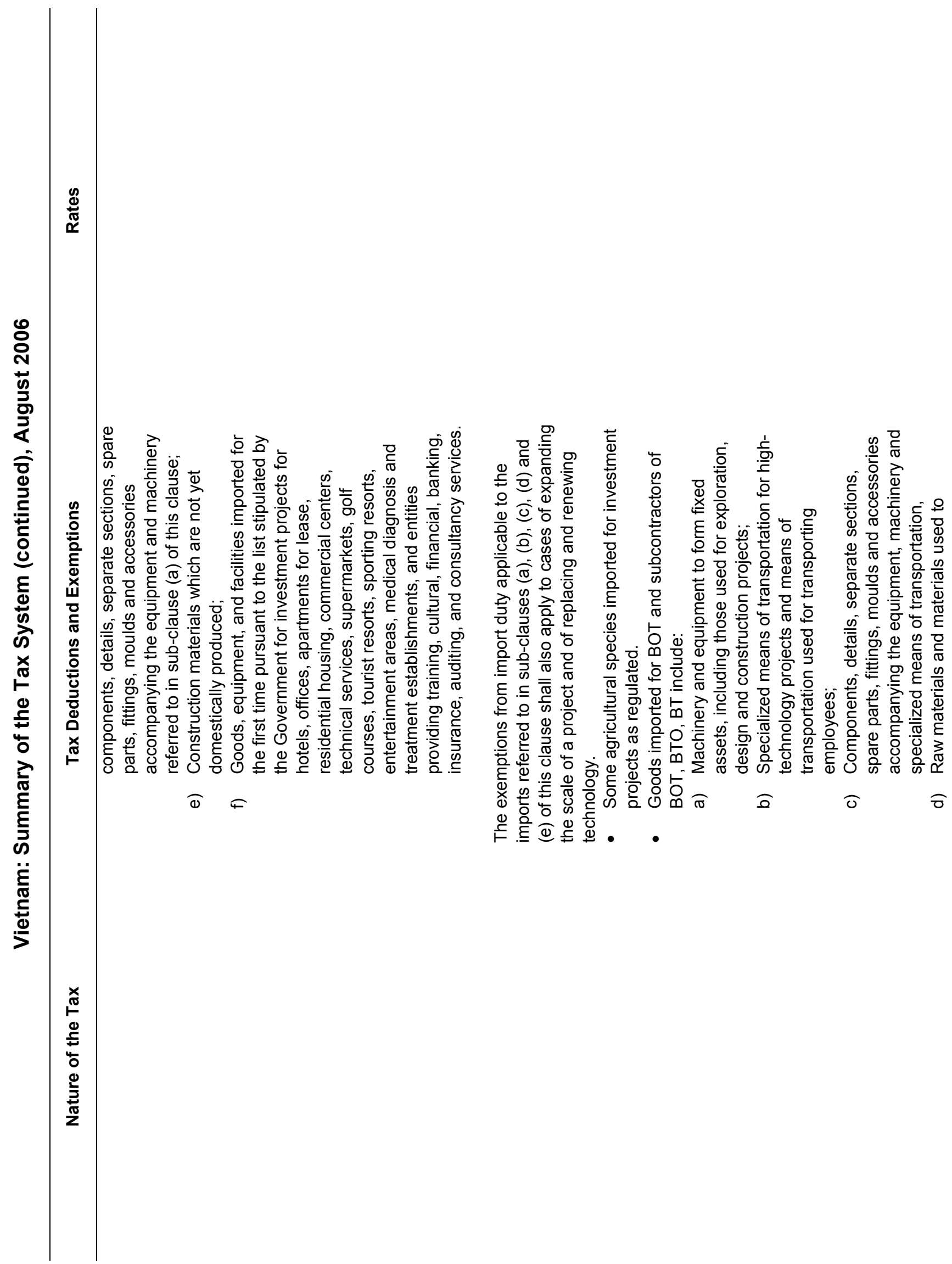


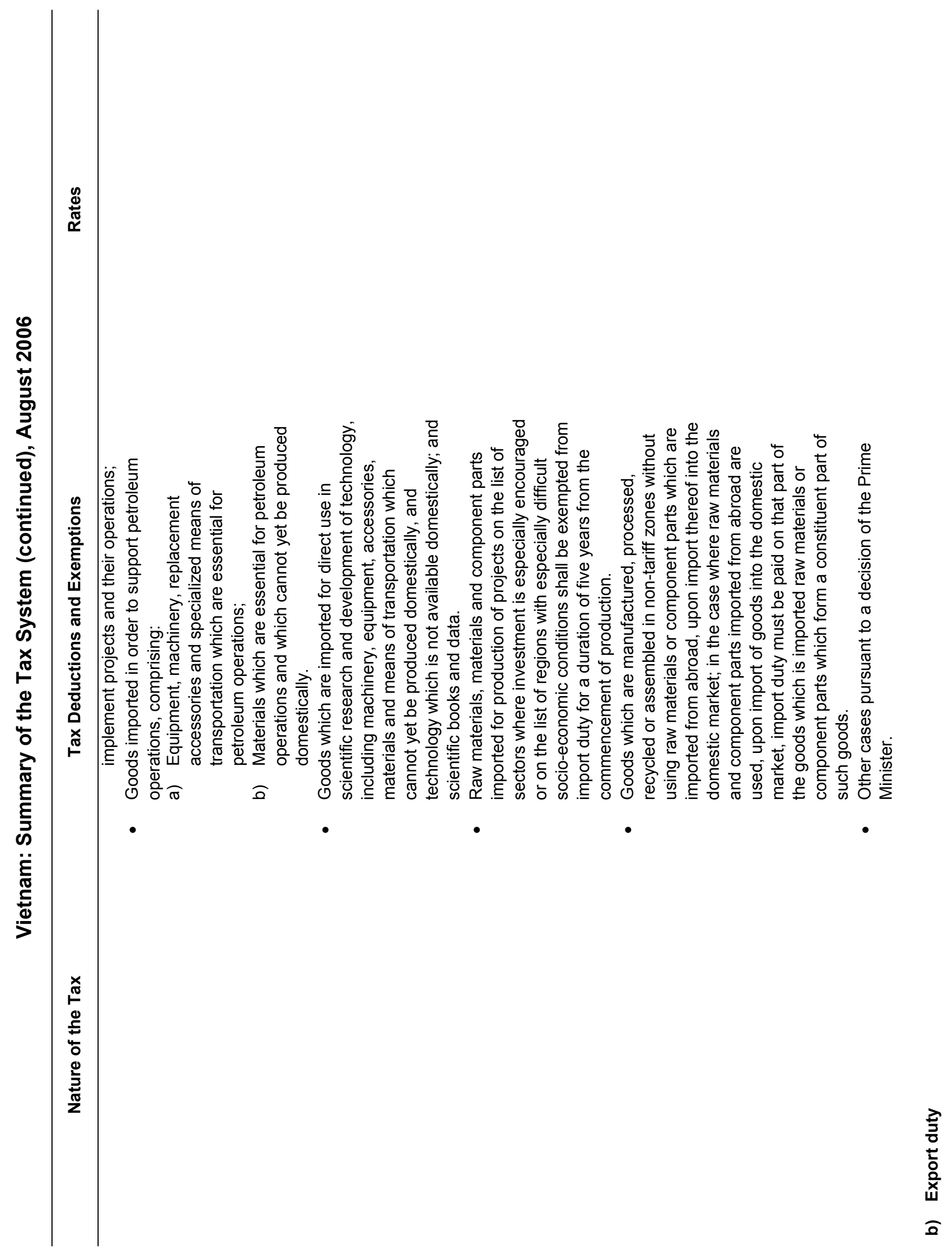




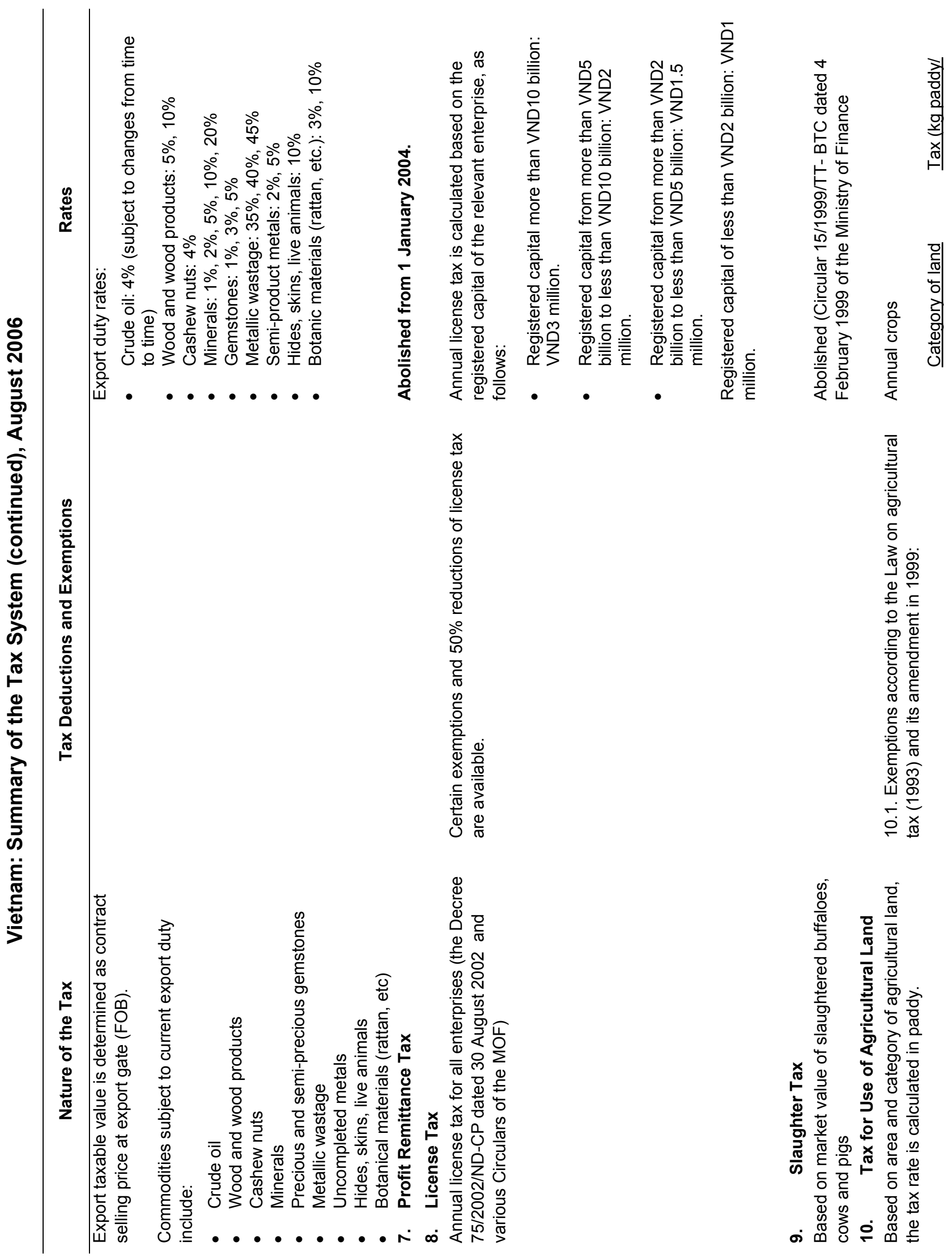




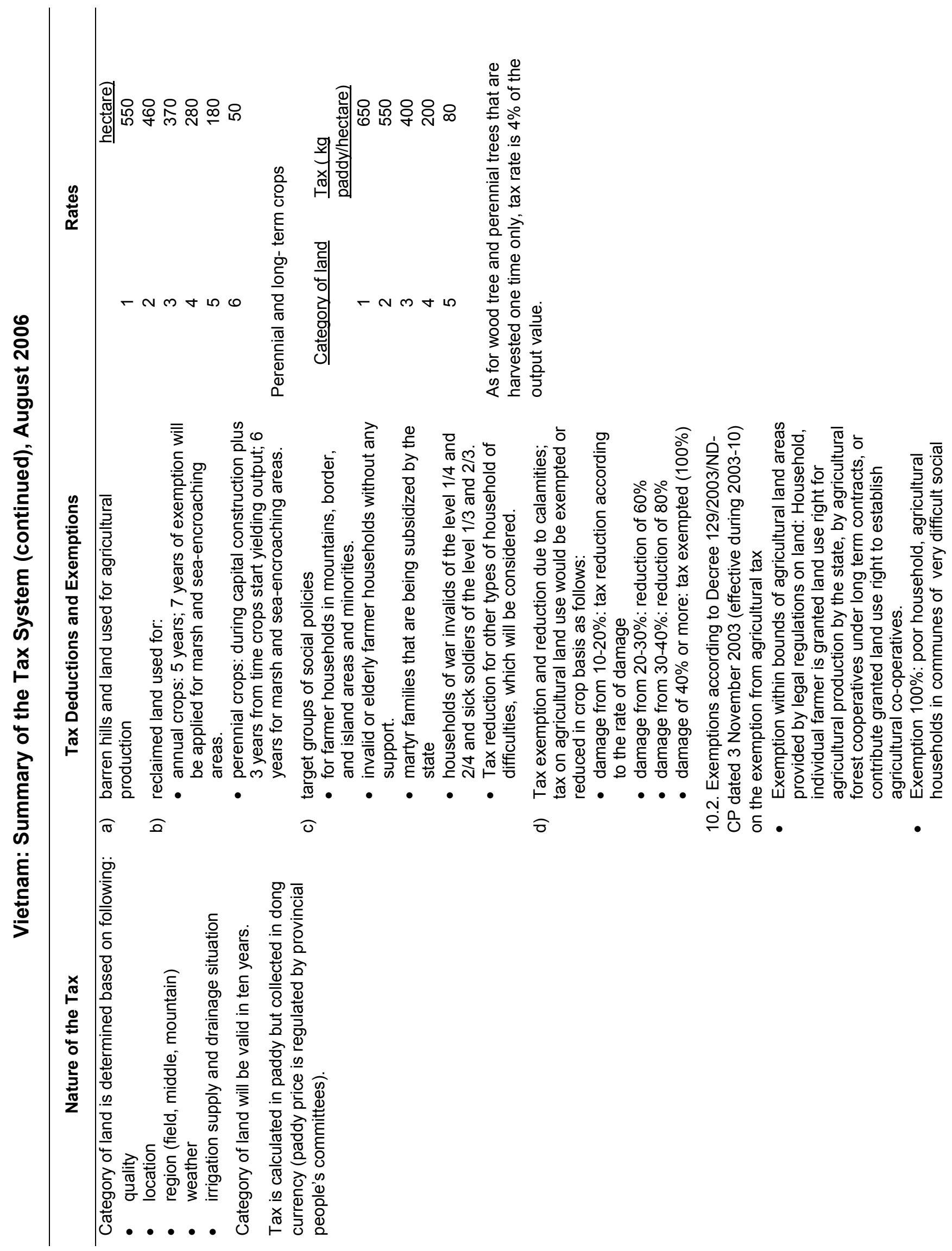




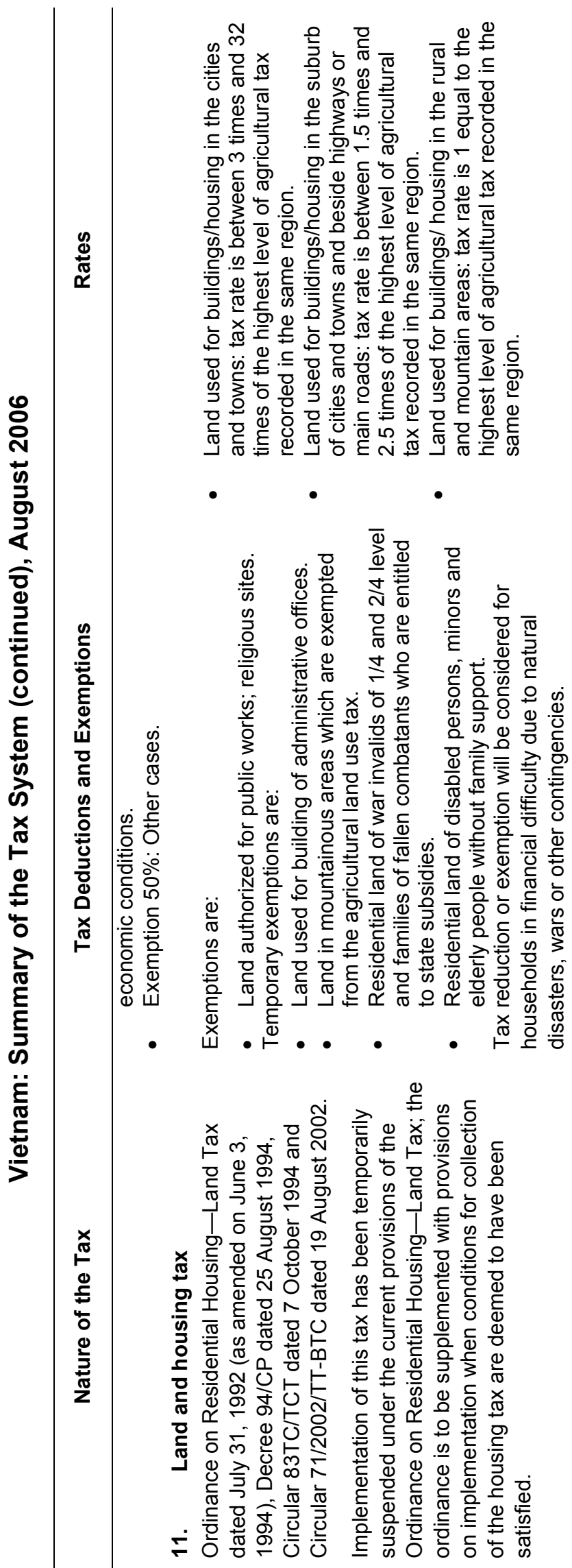

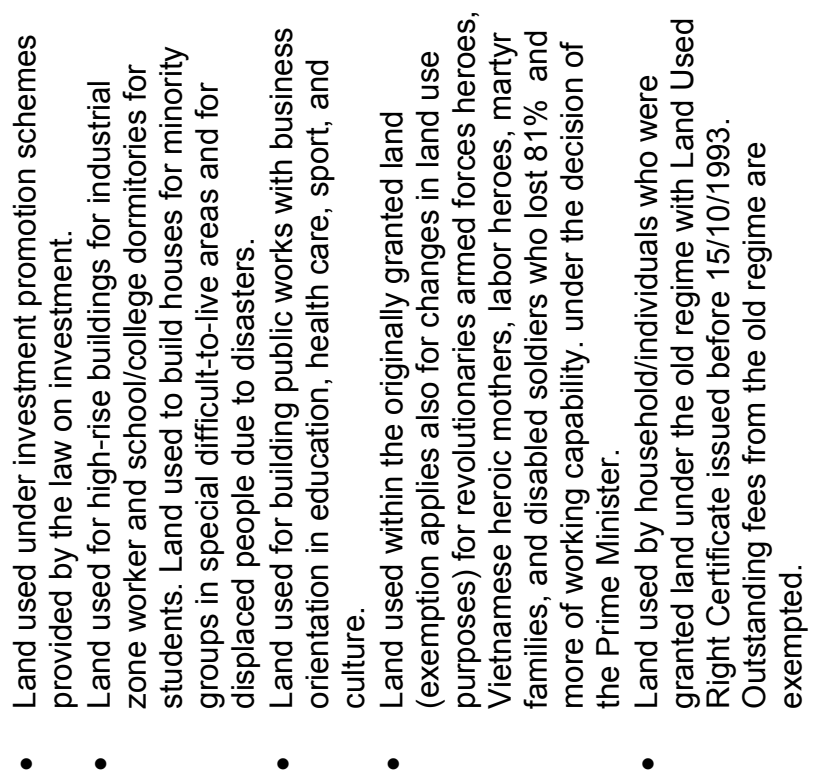

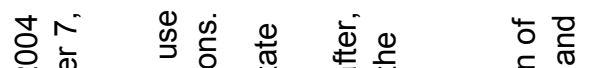

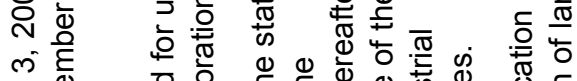

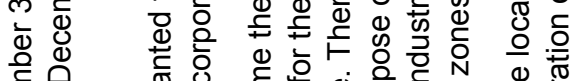

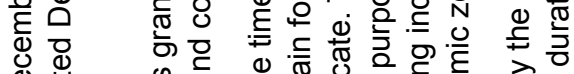

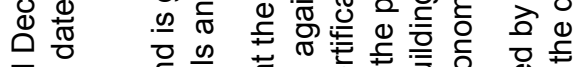

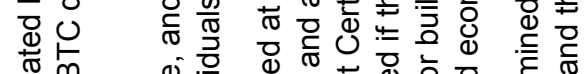

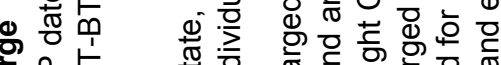

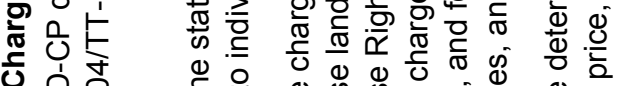

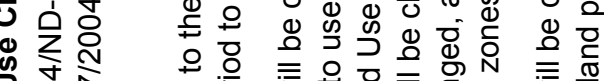

至劣

స్

竞

ヘ 


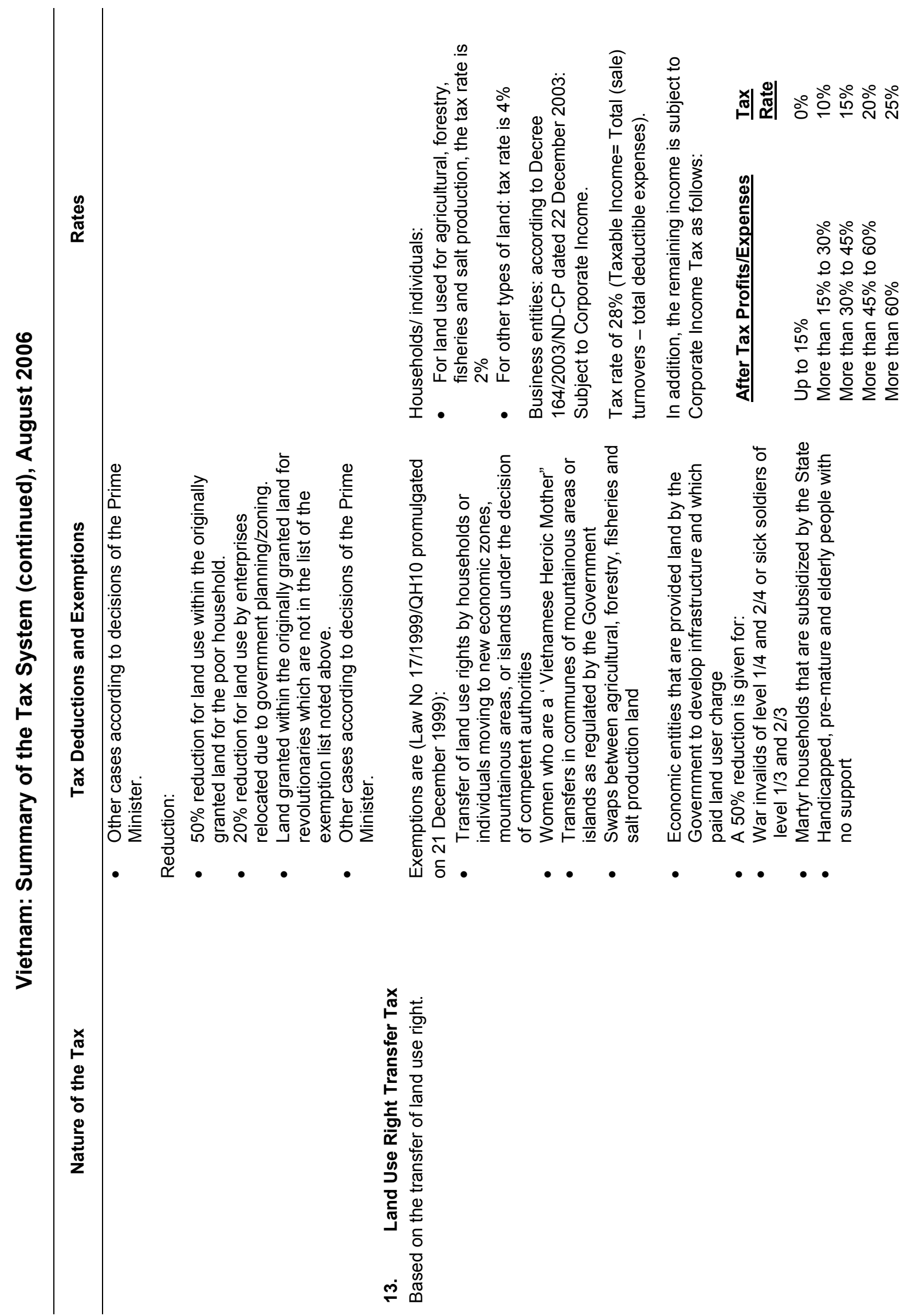




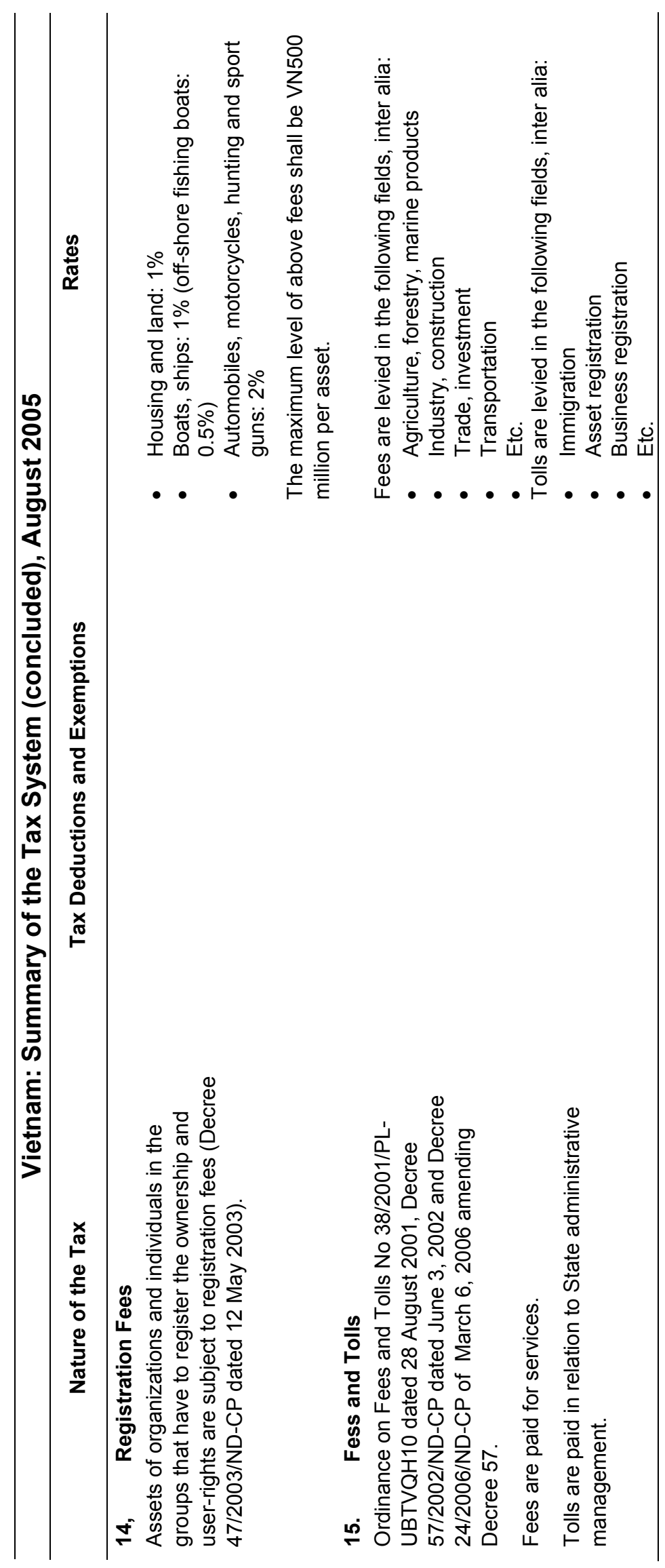

